\title{
MULTIVARIABLE AVERAGING ON SPARSE SETS
}

\author{
P. LAVICTOIRE, A. PARRISH, AND J. ROSENBLATT
}

\begin{abstract}
Nonstandard ergodic averages can be defined for a measure-preserving action of a group on a probability space, as a natural extension of classical (nonstandard) ergodic averages. We extend the one-dimensional theory, obtaining $L^{1}$ pointwise ergodic theorems for several kinds of nonstandard sparse group averages, with a special focus on the group $\mathbb{Z}^{d}$. Namely, we extend results for sparse block averages and sparse random averages to their analogues on virtually nilpotent groups, and extend Christ's result for sparse deterministic sequences to its analogue on $\mathbb{Z}^{d}$. The second and third results have two nontrivial variants on $\mathbb{Z}^{d}$ : a "native" $d$-dimensional average and a "product" average from the one-dimensional averages.
\end{abstract}

\section{Contents}

1. Introduction

1.1. Pointwise ergodic theorems along sparse sets

1.2. Pointwise ergodic theorems over discrete groups

1.3. Sparse averages over discrete groups

1.4. Results

2. Averages along blocks

2.1. A plaid construction

2.2. A divergent construction

3. Multivariable random averages

3.1. Speckled random averages on $\mathbb{Z}^{d}$

3.2. Plaid random averages on $\mathbb{Z}^{d}$

4. Sparse arithmetic sets in $\mathbb{Z}^{d}$

4.1. A speckled construction

4.2. The product construction

5. Sparse sequences and actions of virtually nilpotent groups

5.1. Block averages for virtually nilpotent groups

5.2. Random averages for measure-preserving group actions

5.3. Gaps and Banach density

Acknowledgments

References

\section{INTRODUCTION}

1.1. Pointwise ergodic theorems along sparse sets. Subsequence ergodic theorems have been related to interesting questions since the beginning of the subject:

Received by the editors June 21, 2012.

2010 Mathematics Subject Classification. Primary 37A45. 
for instance, the equidistribution of $\left\{n^{2} \alpha\right\}$ for $\alpha \notin \mathbb{Q}$ corresponds to the mean convergence of the averages along the squares,

$$
\frac{1}{N} \sum_{k=1}^{N} f \circ T^{k^{2}}(x) .
$$

The pointwise theory of nonstandard ergodic averages has an eventful history marked by a combination of "qualitative" methods, characterized by an interest in infinitary properties such as convergence, and "quantitative" techniques, which spring from a more hard-analytical background. In the qualitative direction, Bellow and Losert [2] were the first to construct sparse sequences $\left\{n_{k}\right\}$ for which pointwise ergodic theorems could be proved; these sequences consist of increasingly large blocks of integers separated by increasingly, but not arbitrarily, vast gaps. The pointwise convergence (almost everywhere) of these averages followed from covering lemmas and asymptotic properties of the averaging sets.

A seminal quantitative result was Bourgain's proof [5] that the averages along the squares (1.1) converge pointwise (almost everywhere) as $N \rightarrow \infty$ for any dynamical system $(X, T)$ and any $f \in L^{2}(X)$. This result, and its many subsequent variants, used a transference argument and the Fourier-analytic properties of corresponding exponential sums to prove maximal and oscillational inequalities for the averages.

One distinction between the two methods concerns the endpoint space $L^{1}(X)$. The methods applied in [2] automatically prove convergence for all $f \in L^{1}$, but the Fourier transform methods for various sequences could be extended to $L^{p}, p>1$, only by interpolation. As a result, the $L^{1}$ case for the sequence of squares remained open; for two decades, the sparsity of block sequences remained the only kind of sparsity for which $L^{1}$ pointwise ergodic theorems could be proved.

In recent years, there has been substantial progress on the $L^{1}$ pointwise ergodic theory for sparse averages via a technique of Fefferman [11] and Christ [8] which arises from the theory of singular integrals. Urban and Zienkiewicz 23 used this method to prove an $L^{1}$ pointwise ergodic theorem for the averages along $\left\lfloor n^{\alpha}\right\rfloor$ (for $\alpha$ slightly greater than 1), and LaVictoire [15] proved a similar pointwise theorem for random sequences: specifically that averages taken along a sequence of integers chosen randomly, and nearly as sparse as the sequence of square numbers, must converge pointwise almost surely for $f \in L^{1}$.

However, Buczolich and Mauldin [7] proved that the averages along the squares (1.1) do not satisfy an $L^{1}$ pointwise ergodic theorem; this argument is fundamentally a qualitative proof but uses some quantitative results from number theory. This result, among others, demonstrates that the $L^{1}$ case is distinct from, and requires different approaches compared to, the $L^{p}$ case, $p>1$.

Finally, in 10, Christ has provided new deterministic examples of sparse sequences that allow for pointwise convergence results in $L^{1}$. These sequences are constructed from the sets $\left\{\left(a, a^{2}, \ldots, a^{n}\right) \subset \mathbb{Z}_{p}^{n}: a \in \mathbb{Z}_{p}\right\}$, using Freiman isomorphisms to find sequences of integers which have similar Fourier properties. The Weil bounds on the corresponding exponential sums give optimal Fourier bounds on the averages along such a sequence; these optimal Fourier bounds allow the application of another version of the technique of Fefferman and Christ, which obtains a weak $(1,1)$ maximal inequality.

1.2. Pointwise ergodic theorems over discrete groups. Another classically interesting extension of ergodic theory concerns more general averages obtained 
from a measure-preserving action $\mathcal{T}$ of a discrete group $G$ on a probability space $X$. To each $g \in G$ we associate a measure-preserving transformation $\mathcal{T}(g)$ on $X$, such that $\mathcal{T}(g) \circ \mathcal{T}\left(g^{\prime}\right)=\mathcal{T}\left(g g^{\prime}\right)$. If $G=\mathbb{Z}^{d}$, in particular, a measure-preserving group action consists of $d$ commuting transformations $\left(T_{1}, \ldots, T_{d}\right)$; for each $\vec{n} \in \mathbb{Z}^{d}$, we have the transformation $\mathcal{T}(\vec{n})=T_{1}^{n_{1}} \cdots T_{d}^{n_{d}}$.

Tempelman 22] obtained a fairly general pointwise ergodic theorem (Theorem 2.1) for group actions, defined with respect to a sequence of finite subsets $F_{N} \subset G$. We consider the averages

$$
A_{N} f=\frac{1}{\# F_{N}} \sum_{\vec{n} \in F_{N}} f \circ \mathcal{T}(g) .
$$

If the sequence of sets $\left\{F_{N}\right\}$ satisfies the Tempelman Condition (2.1), then for any $f \in L^{1}(X), A_{N} f$ will converge pointwise for every measure-preserving action $\mathcal{T}$, irrespective of the underlying probability space $X$.

There is a useful class of discrete groups which we will consider in particular:

Definition. A finitely generated group $G$ is virtually nilpotent if it contains a nilpotent subgroup of finite index.

If we consider a finite set $\mathbb{A}$ of generators of a group $G$, and let $\mathbb{A}^{N}$ denote the words of length $\leq N$ in that alphabet, we have that these virtually nilpotent groups are precisely those in which $\# \mathbb{A}^{N}$ grows at a rate polynomial in $N$ (see [16], 26], and [12]). This polynomial growth rate allows for interesting applications of both qualitative and quantitative techniques in the cases of block sequences and random sequences, respectively, ultimately resulting in new pointwise convergence results.

1.3. Sparse averages over discrete groups. Combining the qualitative and quantitative approaches, we study sparse ergodic averages in the group setting and prove $L^{1}$ pointwise convergence results in a number of contexts.

By sparse, we refer to a quality of a set, taken from a group $G$, analogous to that of being zero density in the integers. In the integers, density is determined by comparing a set to nested sets of closed intervals. When we consider virtually nilpotent groups, however, there are more choices for sets to which we can compare ours than in the case $G=\mathbb{Z}$. The sets defined by word-length in the generating set prove to be a good choice; by a result of Pansu [17, the corresponding notion of sparseness is independent of our choice of a generating set.

In addition to this basic idea of sparseness, we will also consider other descriptions of the density of a set. The first is an extension of the idea of upper Banach density; the second is an extension of the condition that gaps tend to $\infty$.

Definition. Let $F$ be a subset of a virtually nilpotent group $G$, and $\mathbb{A}$ a finite generating set. We say $F$ is sparse if

$$
\lim _{N \rightarrow \infty} \frac{\#\left(F \cap \mathbb{A}^{N}\right)}{\# \mathbb{A}^{N}}=0 .
$$

We say that $F$ has Banach density 0 if

$$
\lim _{N \rightarrow \infty} \sup _{g \in G} \frac{\#\left(g F \cap \mathbb{A}^{N}\right)}{\# \mathbb{A}^{N}}=0 .
$$

Finally, we say that $F$ has gaps tending to $\infty$ if, for every $N$, there are only finitely many $g \in F$ such that $g \mathbb{A}^{N} \cap F \neq\{g\}$. 
We will exclude from our consideration sets that are sparse by virtue of essentially being averages over a subgroup of $G$ - for instance the sets $\{1, \ldots, N\} \times\{0\} \subset \mathbb{Z}^{2}$ since these are classical group averages in disguise. That is, we will be looking for sequences of sets $F_{N}$ such that if one takes any sequence of subsets $D_{N} \subset F_{N}$ with $\limsup _{N \rightarrow \infty} \frac{\# D_{N}}{\# F_{n}}=0$, then $\bigcup_{N=1}^{\infty} F_{n} \backslash D_{N}$ still generates the entire group $G$.

1.4. Results. We have three main categories of results, each obtained by a different method. Furthermore, in the case $G=\mathbb{Z}^{d}$, each of these has two variants. Colloquially, we call a construction plaid if it consists of a Cartesian product of sparse subsets of $\mathbb{Z}$, and we call it native if it is built in a genuinely $d$-dimensional manner. It should be noted that the results in the plaid case are still nontrivial, since the averages do not factor. (The associated Fourier transforms do factor, but this helps only in $L^{2}$ and not in $L^{1}$.)

The first category of results, discussed in Section 2 for $\mathbb{Z}^{d}$ and Section 5.1 for virtually nilpotent groups, extends the sparse block sequence result of Bellow and Losert [2] by showing that appropriate sparse sequences of sets satisfy the Tempelman Condition (2.1), and thus Tempelman's Ergodic Theorem (Theorem 2.1) applies. These sets are sparse, but do not have Banach density 0 .

The second category, developed in Sections 3 for $\mathbb{Z}^{d}$ and 5.2 for virtually nilpotent groups, extends the sparse random sequence result of LaVictoire [15]. For simplicity, here we will state only the speckled $\mathbb{Z}^{d}$ version. Let $\left\{\xi_{\vec{n}}: \vec{n} \in \mathbb{Z}^{d}\right\}$ be independent $\{0,1\}$-valued random variables, such that if $2^{j} \leq|\vec{n}|<2^{j+1}$, then $\mathbb{P}\left(\xi_{\vec{n}}=1\right)=$ $2^{-\gamma j} \approx|\vec{n}|^{-\gamma}$.

Then for $\gamma<d / 2$, with probability 1 , the random set generated by $\left\{\xi_{\vec{n}}\right\}$ satisfies an $L^{1}$ pointwise ergodic theorem:

Theorem 1.1. For $\gamma<d / 2$, the following holds for all $\omega \in \Omega$ except for a set of probability 0 :

Let $\left\{\vec{a}_{k}\right\}_{k \in \mathbb{N}}$ be an enumeration of the set $\left\{\vec{n}: \xi_{\vec{n}}(\omega)=1\right\}$ with $\left|\vec{a}_{k}\right|$ increasing. Then for any measure-preserving $\mathbb{Z}^{d}$-action $\mathcal{T}$ and any $f \in L^{1}(X)$, the averages

$$
A_{N} f(x):=\frac{1}{N} \sum_{k=1}^{N} f \circ \mathcal{T}\left(\vec{a}_{k}\right)(x)
$$

converge almost everywhere in $X$.

Additionally, we prove a plaid version on $\mathbb{Z}^{d}$ (Corollary 3.5) and a version on general groups (Theorem [5.6). The latter requires us to first prove an $L^{2}$ ergodic theorem (Theorem 5.5) since the Fourier transform methods which work for $\mathbb{Z}^{d}$ do not work on nonabelian groups. An argument based in combinatorics and the $T T^{*}$ method from harmonic analysis suffices instead.

With probability 1, these random sequences are not only sparse, but have Banach density 0 . Further, each can be modified to a sequence whose gaps tend to $\infty$ and along which an $L^{1}$ pointwise ergodic theorem still holds (Section 5.3).

The third category of results, developed in Section 4, extends the sparse deterministic result of Christ [10] to $\mathbb{Z}^{d}$ with both a native version (Theorem 4.4) and a product version (Proposition 4.7). We will state the simplest form of the first version. 
Theorem 1.2. Let $p_{k}$ be prime numbers with $2^{k}<p_{k}<2^{k+\frac{1}{2}}$. Then if we take the sparse set

$$
S=\bigcup_{k=1}^{\infty} \bigcup_{j=0}^{p_{k}-1}\left(2^{k+1}+[j]_{p_{k}},\left[j^{2}\right]_{p_{k}}, \cdots,\left[j^{d}\right]_{p_{k}}\right)
$$

and order it by first coordinate, the averages along this set converge (almost everywhere) for every measure-preserving $\mathbb{Z}^{d}$-action $\mathcal{T}$ and any $f \in L^{1}(X)$.

The a.e. convergence in this result is new even for the original averages in $\mathbb{Z}$; in [10] only the weak $(1,1)$ maximal inequality is proved. The existence of a dense class for which pointwise convergence holds follows from an oscillational inequality (Theorem 4.3) for the averages, as in Section IV.2 of Rosenblatt and Wierdl's monograph, [18. The pointwise convergence for $L^{1}$ functions follows. These sequences are sparse as well, and can be modified into a sequence whose gaps tend to $\infty$ without affecting the a.e. convergence of the averages.

\section{Averages ALONG BLOCKS}

A sequence of sets $\left\{F_{n}\right\}$ taken from a discrete group $G$ is called a Følner sequence if each set is finite and

for every $g \in G$.

$$
\lim _{n \rightarrow \infty} \frac{\#\left(g F_{n} \triangle F_{n}\right)}{\# F_{n}}=0
$$

A Følner sequence is said to satisfy the Tempelman Condition if there is a constant $C$ so that

$$
\#\left(F_{n}^{-1} F_{n}\right) \leq C \# F_{n} .
$$

Definition. Let $(X, \mathcal{F}, m)$ be a probability space and $\left\{T_{g}: g \in G\right\}$ a group of measure-preserving transformations on $X$ with $T_{g} T_{h}=T_{g h}$ for all $g, h \in G$. We say that $\left\{T_{g}\right\}$ is a measure-preserving group action.

Theorem 2.1 (Tempelman's Ergodic Theorem, 22]). Suppose that $T$ is a measurepreserving action of $G$ on the space $X$ and that $\left\{F_{n}\right\}$ is a nested Følner sequence that satisfies the Tempelman Condition. Then the averages

$$
\frac{1}{\# F_{n}} \sum_{g \in F_{n}} f\left(T_{g} x\right)
$$

converge for a.e. $x \in X$.

In this section we seek a generalized form of the block sequence example of Bellow and Losert [2]. We will examine both a plaid and a speckled version: our method, in each case, will be to show that a suitably chosen Følner sequence satisfies the Tempelman Condition, (2.1). We note here that while each construction in this section will have zero density, neither are of Banach density 0. Much like the original construction, the existence of the block-like structures does not allow for this type of sparseness. Further, in the first construction, we consider only two variable free actions. The ideas presented, however, will work in any finite number of variables. 
2.1. A plaid construction. In this construction, our aim is to build a sparse sequence of sets by considering the products of one-dimensional sparse sequences. Among the difficulties in two dimensions is the loss of a natural order in which to take our averages; hence we must not only define our larger set, whence we derive sparseness, but also an ordering of its elements. This ordering will give us our sequence of sets.

We start with a sequence of rectangles $\left\{D_{k}\right\}$ in $\mathbb{Z}^{2}$, where each $D_{k}=R_{k}+\left(u_{k}, v_{k}\right)$ and $R_{k}=\left[1, a_{k}\right] \times\left[1, b_{k}\right]$. We will need to have these rectangles well spaced, so we will assume that

$$
u_{k+1} \geq u_{k}+a_{k} \text { and } v_{k+1} \geq v_{k}+b_{k} .
$$

We also want to arrange for these rectangles to provide us with projections along the axes that give sequences of zero density. So we will also assume that

$$
\frac{\sum_{i=1}^{k-1} a_{i}}{a_{k}} \rightarrow 0 \text { and } \frac{\sum_{j=1}^{k-1} b_{j}}{b_{k}} \rightarrow 0 \text { as } k \rightarrow \infty .
$$

It follows from equation (2.3) that $a_{k} \rightarrow \infty$ and $b_{k} \rightarrow \infty$ as $k \rightarrow \infty$.

An additional regularity assumption will be necessary in order to prove almost everywhere convergence results. It is that for some constant $C>0$, we have

$$
a_{k} \geq C u_{k-1} \text { and } b_{k} \geq C v_{k-1} \text {. }
$$

This assumption is one of the conditions used by Bellow and Losert in [2], and by Bellow, Jones, and Rosenblatt in 3 .

We want to use this sequence of blocks to form unions of their projections on the coordinate axes, and then form two variable averaging operators by putting these back together. We denote the union of the projections of the blocks $\left\{D_{1}, \ldots, D_{k}\right\}$ on the coordinate axes by $A(k)=\bigcup_{i=1}^{k}\left[u_{i}+1, u_{i}+a_{i}\right]$ and $B(k)=\bigcup_{i=1}^{k}\left[v_{i}+1, v_{i}+b_{i}\right]$. We also want to work with the intermediate blocks that come from the projections, so for each $k$ and $r=1, \ldots, a_{k}$, we denote them by $A(k, r)=A(k-1) \cup\left[u_{k}+1, u_{k}+r\right]$ and $B(k, r)=B(k-1) \cup\left[v_{k}+1, v_{k}+r\right]$. Note that $A\left(k, a_{k}\right)=A(k)$ and $B\left(k, b_{k}\right)=$ $B(k)$.

The first fact to observe from [3] is that the sequences of sets $\{A(k)\}$ and $\{B(k)\}$ satisfy the Cone Condition: thus the operators $\mathcal{A}_{k} f=\frac{1}{a_{k}} \sum_{i=1}^{a_{k}} f \circ S^{u_{k}+i}$ and $\mathcal{B}_{k} f=$ $\frac{1}{b_{k}} \sum_{j=1}^{b_{k}} f \circ T^{v_{k}+j}$ have maximal functions that are weak $(1,1)$ and strong $(p, p)$ for all $p, 1<p<\infty$. Since $a_{k} \rightarrow \infty$ and $b_{k} \rightarrow \infty$, we get pointwise and norm convergence to the projection of the invariant functions for all $L^{p}, 1 \leq p<\infty$, in each case.

Also, as implicit in [2] without proof, the intermediate sets $\{A(k, r): k \geq 1,1 \leq$ $\left.r \leq a_{k}\right\}$ and $\left\{B(k, r): k \geq 1,1 \leq r \leq b_{k}\right\}$ are Følner sets that satisfy (2.1) (see Proposition 2.2). In particular, there is a constant such that $\#(A(k, r)-A(k, r)) \leq$ $C \# A(k, r)$ and $\#(B(k, r)-B(k, r)) \leq C \# B(k, r)$ for all $k$ and $r$. As a result, the operators $\mathcal{A}(k, r) f=\frac{1}{\# A(k, r)} \sum_{i \in A(k, r)} f \circ S^{i}$ and $\mathcal{B}(k, r) f=\frac{1}{\# B(k, r)} \sum_{j \in B(k, r)} f \circ T^{j}$ have maximal functions that are weak $(1,1)$ and strong $(p, p)$ for all $p, 1<p<\infty$. (See Tempelman, 22.) So again we get pointwise and norm convergence to the projection of the invariant functions in each case, for all $L^{p}, 1 \leq p<\infty$. The 
pointwise convergence result is clear from the Cone Condition if one were restricting oneself to the ends of the individual block (i.e. using only $A(k)=A\left(k, a_{k}\right)$ and $\left.B(k)=B\left(k, b_{k}\right)\right)$. But this result actually requires the following computation even for these sequences, let alone the full sequence of intermediate sets. We give this proof because it does not appear in [2] and there are some not necessarily obvious details that do need to be considered.

Proposition 2.2. The sequences of sets $\{A(k, r)\}$ and $\{B(k, r)\}$ satisfy the Tempelman's regularity conditions.

Proof. We will consider only $\{A(k, r)\}$ since the argument for $\{B(k, r)\}$ is similar. Our sets are all finite. Also, any $A\left(k, r_{1}\right) \subset A\left(k+1, r_{2}\right)$ if $1 \leq r_{1} \leq k$ and $1 \leq r_{2} \leq k+1$, and $A\left(k, r_{1}\right) \subset A\left(k, r_{2}\right)$ if $1 \leq r_{1} \leq r_{2} \leq k$. Because these sets are unions of blocks $\left[u_{i}+1, u_{i}+a_{i}\right]$ and an intermediate block, with the lengths $a_{i} \rightarrow \infty$ as $i \rightarrow \infty$, it is clear that the sequence $(A(k, r))$ satisfies the Følner condition. So the only condition remaining is the fact that there is a constant $M$ so that $\#(A(k, r)-A(k, r)) \leq M \# A(k, r)$ for all $k$ and $r$.

Here we have

$$
\begin{aligned}
A(k, r)-A(k, r) & =\bigcup_{i=1}^{k-1} A_{i} \cup\left[u_{k}+1, u_{k}+r\right]-\bigcup_{i=1}^{k-1} A_{i} \cup\left[u_{k}+1, u_{k}+r\right] \\
& =\left(\bigcup_{i=1}^{k-1} A_{i}-\bigcup_{i=1}^{k-1} A_{i}\right) \cup\left(\bigcup_{i=1}^{k-1} A_{i}-\left[u_{k}+1, u_{k}+r\right]\right) \\
& \cup\left(\left[u_{k}+1, u_{k}+r\right]-\bigcup_{i=1}^{k-1} A_{i}\right) \cup\left[u_{k}+1, u_{k}+r\right]-\left[u_{k}+1, u_{k}+r\right] .
\end{aligned}
$$

So, as for the size of the set of differences, we are left with

$$
\begin{aligned}
\#(A(k, r)-A(k, r)) \leq & \#\left(\bigcup_{i=1}^{k-1} A_{i}-\bigcup_{i=1}^{k-1} A_{I}\right) \\
& +2 \#\left(\bigcup_{i=1}^{k-1} A_{i}-\left[u_{k}+1, u_{k}+r\right]\right)+2 r-1
\end{aligned}
$$

By Lemma 3.2 of [2], we know that $\bigcup_{i=1}^{k-1} A_{i}$ satisfies all of the requirements of Tempelman's Theorem. In particular, this means there is then a constant $M_{1}$ so that

$$
\#\left(\bigcup_{i=1}^{k-1} A_{i}-\bigcup_{i=1}^{k-1} A_{i}\right) \leq M_{1} \#\left(\bigcup_{i=1}^{k-1} A_{i}\right)=M_{1} \sum_{i=1}^{k-1} a_{i}
$$

Combining the assumptions above with our estimate, we then find that there is a constant $M_{2}$ so that

$$
\#\left(\bigcup_{i=1}^{k-1} A_{i}-\bigcup_{i=1}^{k-1} A_{i}\right) \leq M_{2} a_{k-1}
$$


We now will turn our attention to \# $\left(\bigcup_{i=1}^{k-1} A_{i}-\left[u_{k}+1, u_{k}+r\right]\right)$ :

$$
\begin{aligned}
& \bigcup_{i=1}^{k-1} A_{i}-\left[u_{k}+1, u_{k}+r\right] \\
& =\left(\bigcup_{i=1}^{k-2} A_{i}-\left[u_{k}+1, u_{k}+r\right]\right) \cup\left(A_{k-1}-\left[u_{k}+1, u_{k}+r\right]\right) \\
& \subseteq\left[-u_{k}-r, u_{k-2}+a_{k-2}-u_{k}-1\right] \cup\left[u_{k-1}-u_{k}-r, u_{k-1}+a_{k-1}-u_{k}\right] .
\end{aligned}
$$

Looking at the lengths of these intervals, we find that

$$
\#\left(\bigcup_{i=1}^{k-1} A_{i}-\left[u_{k}+1, u_{k}+r\right]\right) \leq u_{k-2}+a_{k-2}+a_{k-1}+2 r+1 .
$$

But from our requirement relating the growth of $\left(u_{k}\right)$ and $\left(a_{k}\right)$, we know that there is a nonzero constant $M_{3}$ so that

$$
u_{k-2}+a_{k-2} \leq M_{3} a_{k-1} .
$$

So we have that

$$
\#\left(\bigcup_{i=1}^{k-1} A_{i}-\left[u_{k}, u_{k}+r\right]\right) \leq\left(M_{3}+1\right) a_{k-1}+2 r+1 .
$$

Combining the inequalities (2.6) and (2.7), we find that there are constants $M_{4}$ and $M_{5}$ so that

$$
\#(A(r, k)-A(r, k)) \leq M_{4} a_{k-1}+M_{5} r .
$$

Now, suppose $r \leq a_{k-1}$. Then we have that

$$
\#(A(k, r)-A(k, r)) \leq\left(M_{4}+M_{5}\right) a_{k-1} .
$$

But \# $A(k, r)$ must be more than $a_{k-1}$ since $A(k, r)$ contains the $k-1$ block. So

$$
\#(A(k, r)-A(k, r)) \leq\left(M_{4}+M_{5}\right) \# A(k, r) .
$$

If, on the other hand, we have $r>a_{k-1}$, then

$$
\#(A(k, r)-A(k, r))<\left(M_{4}+M_{5}\right) r .
$$

But $\# A(k, r)$ is more than $r$ because $A(k, r)$ contains the set $\left[u_{k}+1, u_{k}+r\right]$. So again

$$
\#(A(k, r)-A(k, r)) \leq\left(M_{4}+M_{5}\right) \# A(k, r)
$$

We now want to put the sequences $\{A(k, r)\}$ and $\{B(k, r)\}$ back together. Suppose we write $\bigcup_{(k, r)} A(k, r)=\left\{s_{m}\right\}$ and $\bigcup_{(k, r)} B(k, r)=\left\{t_{n}\right\}$ where $\mathbf{s}=\left\{s_{m}\right\}$ and $\mathbf{t}=\left\{t_{n}\right\}$ are increasing sequences. Then take the commuting maps $S$ and $T$ and consider the two variable averages

$$
\mathcal{A}_{(M, N)} f=\frac{1}{M N} \sum_{m=1}^{M} \sum_{n=1}^{N} f \circ S^{s_{m}} \circ T^{t_{n}} .
$$


Proposition 2.3. The sequence $\left\{\mathcal{A}_{(N, N)}\right\}$ has a maximal function that is weak $(1,1)$ and strong $(p, p)$. Hence, for an ergodic $\mathbb{Z}^{2}$-action, for all $f \in L^{1}(X)$, we have $\lim _{N \rightarrow \infty} \mathcal{A}_{(N, N)} f(x)=\int_{X} f d m$.

Proof. Each $A_{(N, N)}$ corresponds to an average over a set of the form $A\left(k_{1}, r_{1}\right) \times$ $B\left(k_{2}, r_{2}\right)$. Because we have taken $M=N$ here, these sets are nested. Also, these sets satisfy the Følner condition. So, we obtain our result from Tempelman's Theorem 22 if we have a constant $C_{o}$ such that

$$
\#\left(A\left(k_{1}, r_{1}\right) \times B\left(k_{2}, r_{2}\right)-A\left(k_{1}, r_{1}\right) \times B\left(k_{2}, r_{2}\right)\right) \leq C_{o} \#\left(A\left(k_{1}, r_{1}\right) \times B\left(k_{2}, r_{2}\right)\right) \text {. }
$$

But

$$
\begin{aligned}
& \#\left(A\left(k_{1}, r_{1}\right) \times B\left(k_{2}, r_{2}\right)-A\left(k_{1}, r_{1}\right) \times B\left(k_{2}, r_{2}\right)\right) \\
& =\#\left(\left(A\left(k_{1}, r_{1}\right)-A\left(k_{1}, r_{1}\right)\right) \times\left(B\left(k_{2}, r_{2}\right)-B\left(k_{2}, r_{2}\right)\right)\right) \\
& =\#\left(A\left(k_{1}, r_{1}\right)-A\left(k_{1}, r_{1}\right)\right) \#\left(B\left(k_{2}, r_{2}\right)-B\left(k_{2}, r_{2}\right)\right) \\
& \leq C^{2} \# A\left(k_{1}, r_{1}\right) \# B\left(k_{2}, r_{2}\right) \\
& =C^{2} \#\left(A\left(k_{1}, r_{1}\right) \times B\left(k_{2}, r_{2}\right)\right) .
\end{aligned}
$$

The result above is a two variable ergodic theorem with averaging over sets that have density zero along all horizontal and vertical lines in $\mathbb{Z}^{2}$.

We would like to turn this block method into a sequence method as appears in Theorem 1.1 and Corollary 3.5. But we do not yet know how to amalgate enumerations of the supports of $\mathcal{A}_{(N, N)}$ to achieve this.

We would like to know more about integrability of the maximal function $\sup _{N \geq 1}\left|\mathcal{A}_{(N, N)} f\right|$. But first we have this basic question.

Question. Do the maximal functions of $\mathcal{A}(k, r) f$ and $\mathcal{B}(k, r) f \operatorname{map} L \log L$ to $L^{1}$ ?

It seems plausible that the answer to this question is affirmative because of the following fact:

Proposition 2.4. The maximal functions for $\mathcal{A}_{k} f$ and $\mathcal{B}_{k} f$ map $L \log L$ to $L^{1}$.

Proof. We prove this result just for $\mathcal{A}_{k}^{*} f$, the maximal function of $\mathcal{A}_{k} f$, because the proof for $\mathcal{B}_{k} f$ is identical. We refer to the notation and argument in the proof of Theorem 1, (a) in [3]. Here $C$ denotes an absolute constant, but not necessarily the same constant throughout. Let $f^{*}=\sup _{k} \frac{1}{2 k+1} \sum_{j=-k}^{k}\left|f\left(T^{j} x\right)\right|$, a two sided version of the classical maximal function. The classical result for $f \in L \log L$ gives the inequality $\left\|f^{*}\right\|_{1} \leq C \int_{X}|f|\left(1+\log ^{+}|f|\right) d m$.

Given the Cone Condition, there is a constant $C$ such that for all $\phi \in l_{1}(\mathbb{Z})$,

$$
\#\left\{j: \mathcal{A}_{k}^{*} \phi(j)>2 \lambda\right\} \leq C \#\left\{j: \phi^{*}(j)>\lambda\right\} .
$$

This can be seen, with a slight change of notation so as to be consistent with the notation here, by using $\left(B_{i}\right)$, defined on p. 45 in [3], and the inequality $\#\left\{j \mid M_{\Omega} \phi(j)>2 \lambda\right\} \leq C\left|\cup B_{i}\right|$, on p. 46 in 3 .

By Calderón's transfer principle, this gives

$$
m\left\{x: \mathcal{A}_{k}^{*} f(x)>2 \lambda\right\} \leq C m\left\{x: f^{*}(x)>\lambda\right\} .
$$


But then we have

$$
\begin{aligned}
\left\|\mathcal{A}_{k}^{*} f\right\|_{1} & \leq C \sum_{n=1}^{\infty} m\left\{x: \mathcal{A}_{k}^{*} f>2 n\right\} \\
& \leq C \sum_{n=1}^{\infty} m\left\{x: f^{*}>n\right\} \\
& \leq C\left\|f^{*}\right\|_{1} \\
& \leq C \int_{X}|f|\left(1+\log ^{+}|f|\right) d m .
\end{aligned}
$$

Remark. More generally, one might ask how the maximal functions of $\mathcal{A}(k, r) f$ and $\mathcal{B}(k, r) f$ behave on general Orlicz spaces. In particular, for which Orlicz functions $\Psi_{1}$ and $\Psi_{2}$ do these maximal functions map $\Psi_{1}(L)$ to $\Psi_{2}(L)$ ?

Question. Does the maximal function of $\left(A_{(N, N)} f\right)$ map $L \log ^{2} L$ to $L^{1}$ ? This seems the correct choice of the domain for this result because the maximal function over the first variable should be mapping $L \log ^{2} L$ to $L \log L$.

To obtain pointwise convergence results on $L^{1}(X)$, we needed to restrict our two variable averages $\mathcal{A}(M, N)$ to just using $\mathcal{A}_{(N, N)} f$. While any nested sequence of rectangles would serve as well, there is a good reason to avoid a sequence of sets in which the side lengths are unrelated.

Proposition 2.5. Suppose $S$ and $T$ commute and generate a free ergodic action. Then there exists a function $f \in L^{1}(X), f \geq 0$, such that $\sup _{(M, N)} A_{(M, N)} f=\infty$ a.e.

Proof. Otherwise, by Sawyer's principle (see Sawyer, [19]), there is a weak inequality

$$
m\left\{x \in X: \sup _{(M, N)}\left|A_{(M, N)} f\right|>\lambda\right\} \leq \frac{C}{\lambda}\|f\|_{1}
$$

for all $f \in L^{1}(X)$. Now for $\phi \in \ell^{1}\left(\mathbb{Z}^{2}\right)$, let

$$
A_{(M, N)} \phi(i, j)=\frac{1}{M N} \sum_{m=1}^{M} \sum_{n=1}^{N} \phi\left(s_{m}+i, t_{n}+j\right) .
$$

Because the action is a free ergodic action, our weak inequality transfers to a weak inequality of the form

$$
\#\left\{(i, j) \in \mathbb{Z}^{2}: \sup _{(M, N)}\left|A_{(M, N)} \phi(i, j)\right|>\lambda\right\} \leq \frac{C}{\lambda}\|\phi\|_{1}
$$

for all $\phi \in \ell^{1}\left(\mathbb{Z}^{2}\right)$. Now take the function $\phi=\delta_{(0,0)}$. We then consider the set $E_{n}=$ $\left\{\left(s_{r}, t_{s}\right): r s \leq n\right\}$. Given any $(i, j) \in E_{n}$, we have $\sup _{(M, N)} A_{(M, N)} \phi(i, j) \geq \frac{1}{r s} \geq \frac{1}{n}$. 
Hence, with $\lambda=\frac{1}{n}$, we have

$$
\begin{aligned}
C L & =\frac{C}{1 / n}\|\phi\|_{1} \\
& \geq \#\left\{(i, j) \in \mathbb{Z}^{2}: \sup _{M<N} A_{(M, N)} \phi(i, j)>\frac{1}{n}\right\} \\
& \geq \# E_{n} \\
& =\#\left\{\left(s_{r}, t_{s}\right): r s \leq n\right\} \\
& \geq c n \log n .
\end{aligned}
$$

Letting $n$ tend to infinity gives a contradiction.

Remark. The divergence of the maximal function precludes there being a pointwise a.e. convergence result on $L^{1}(X)$. Also, notice here that it is not necessary to transfer the inequality, just cleaner to state the idea. One could just work with large square Rokhlin towers of height and width $n$ constructed within $X$ and use $f=1_{B}$, where $B$ is the base of the Rokhlin tower. A similar argument to the last string of inequalities will lead to a contradiction as $n \rightarrow \infty$.

Suppose now instead that we have an Orlicz space $\Psi(L)$ with $\Psi$ some regular Orlicz function. Both Stein [20] and Sawyer [19] give useful results concerning maximal inequalities for Orlicz spaces. We will need to have a regular Orlicz function, i.e. one such that $\|\Psi(|f|)\|_{1}$ and the norm $\|f\|_{\Psi(L)}$ are proportional to one another. Besides the usual properties of Orlicz functions, regularity means that for some constant $K$, we have $\Psi(2 x) \leq K \Psi(x)$ for all $x>0$.

We can use the results of [19] to prove the following.

Proposition 2.6. Suppose $S$ and $T$ generate a free ergodic commuting action. If

$$
\sup _{(M, N)}\left|A_{(M, N)} f\right|<\infty
$$

for all $f \in \Psi(L)$, then there exists a constant $C$ such that for all $f \in \Psi(L)$, we have for all $\lambda>0$,

$$
m\left\{x: \sup _{(M, N)}\left|A_{(M, N)} f\right| \geq \lambda\right\} \leq C\left\|\Psi\left(\frac{1}{\lambda}|f|\right)\right\|_{1} .
$$

Otherwise, for a residual set of functions $f \in \Psi(L)$, we have $\sup _{(M, N)}\left|A_{(M, N)} f\right|=\infty$ a.e.

Proof. See Theorems 3 and 4 in [19].

This gives the following.

Proposition 2.7. Suppose $S$ and $T$ generate a free ergodic commuting action. Suppose that $\Psi$ is a regular Orlicz function such that $\Psi(x)=o\left(x \log ^{+} x\right)$ as $x \rightarrow \infty$. Then there exists $f \in \Psi(L)$ such that $\sup _{(M, N)}\left|A_{(M, N)}\right|=\infty$ a.e.

$$
(M, N)
$$

Proof. By Proposition 2.6, if such an $f$ did not exist, then we must have the weak inequality in the proposition. So take the function $f=N 1_{B}$ where $B$ is the base of a square Rokhlin tower of height and width $n$ given by the free action determined 
by $S$ and $T$. We let $\lambda=\frac{1}{n}$. Then we have

$$
\begin{aligned}
C \Psi(n) m(B) & =C\left\|\Psi\left(L 1_{B}\right)\right\|_{1} \\
& \geq m\left\{x: \sup _{(M, N)} A_{(M, N)} 1_{B} \geq \frac{1}{n}\right\} \\
& \geq c n \log n m(B) .
\end{aligned}
$$

Letting $n \rightarrow \infty$ gives a contradiction.

Remark. There is perhaps a generalization of the above to other types of averaging besides Cesàro averaging. Indeed, suppose $\left(\mu_{M}\right)$ and $\left(\nu_{N}\right)$ are two uniformly dissipative averaging methods on $\mathbb{Z}$. Consider the two variable average $A_{(M, N)} f=$ $\sum_{m \in \mathbb{Z}} \sum_{n \in \mathbb{Z}} \mu_{M}(m) \nu_{N}(n) f \circ S^{m} \circ T^{n}$. We conjecture that for a free ergodic commuting action, there would exist a function $f \geq 0$ such that $\sup _{(M, N)} A_{(M, N)} f=\infty$. a.e. As for positive results in this generality, we do not have results at this time.

But in any case we do know that on $L \log L$ the averages $\mathcal{A}_{(M, N)}$ are well behaved.

Proposition 2.8. On $L \log L$, and hence on any $L^{p}(X), 1<p<\infty$, the averages $\mathcal{A}_{(M, N)} f$ converge a.e. for all $f$.

It is clear that the positive results above can be naturally extended to actions of $\mathbb{Z}^{d}$ with $d \geq 3$ too. There will be analogous issues, though, on which Orlicz spaces are best to use when considering unrestricted multivariable averages.

2.2. A divergent construction. In the following section $B_{r}$ will denote the ball of radius $r$ in $\mathbb{Z}^{d}$, and $B_{r}^{+}$will denote that part of the ball consisting of elements all of whose coordinate entries are positive. Similarly,

$$
\mathbb{Z}_{+}^{d}=\left\{\left(a_{1}, a_{2}, \ldots, a_{d}\right) \in \mathbb{Z}^{d} \mid a_{i} \geq 0 \text { for all } i \geq 1\right\} .
$$

Let $I_{k}$ be a sequence of rectangular prisms in $\mathbb{Z}_{+}^{d}$ with one corner at the origin, each having diameter $\ell_{k}$. We also require that the dimensions $b_{1}, b_{2}, \ldots, b_{k}$ of each prism satisfy

$$
c \leq \frac{b_{i}}{b_{j}} \leq C
$$

where $c$ and $C$ are positive, absolute constants.

Suppose these diameters satisfy

$$
\frac{\sum_{i=1}^{k} \ell_{i}}{\ell_{k+1}} \rightarrow 0
$$

as $k \rightarrow \infty$.

Further, suppose we have a sequence of vectors, $\vec{a}_{k} \in \mathbb{Z}_{+}^{d}$, so that

$$
\begin{aligned}
& \left|\vec{a}_{k+1}\right|>\left|\vec{a}_{k}\right|+\ell_{k}, \text { and } \\
& \ell_{k} \geq C\left|\vec{a}_{k-1}\right|,
\end{aligned}
$$

where $C$ is some constant independent of $k$.

Let $S=\bigcup_{k}\left(\vec{a}_{k}+I_{k}\right)$.

We might like to add points to our average in an order depending only on their distance from the origin, i.e. use the sets $\left\{S \cap B_{r}: r \geq 1\right\}$. However, since the ball of radius $r$ is very "flat" in the directions of the coordinate axes, we will either have to use a different ordering or place restrictions on the locations of the blocks. 
Take, for example, the set in $\mathbb{Z}^{2}, S=\bigcup_{k}\left(\vec{a}_{k}+I_{k}\right)$, where, in addition to the requirements above, we have that

(1) $I_{k}$ are squares with diameters $\ell_{k}$,

(2) $\ell_{k}^{2} \leq\left|\vec{a}_{k}\right|$,

(3) $\frac{\sqrt{2}}{2} \ell_{k+1}>\left(\sum_{i=1}^{k} \ell_{i}^{2}\right)^{2}$, and

(4) $\vec{a}_{k}=\left(a_{k}, 0\right)$.

Define the subsequence $\left\{S \cap B_{r_{k}}: r_{k}=\sqrt{\left|\vec{a}_{k}\right|^{2}+\frac{1}{2} \ell_{k}^{2}}\right\}$. This sequence consists of sets comprised of all blocks before the $k$ th, and the left face of the $k$ th block.

Define $f_{k}(x, y): \mathbb{Z}^{2} \rightarrow \mathbb{R}$ by

$$
f_{k}(x, y)= \begin{cases}1 & \text { for } x=a_{k}, 0 \leq y \leq \frac{\sqrt{2}}{2} \ell_{k}, \\ 0 & \text { otherwise }\end{cases}
$$

and let $f(x, y)=\sum_{k} f_{k}(x, y)$.

We then have that the average over $\left\{S \cap B_{r_{k}}\right\}$ is

$$
\frac{1}{\# S \cap B_{r_{k}}} \sum_{(x, y) \in S \cap B_{r_{k}}} f(x, y)>\frac{\frac{\sqrt{2}}{2} \ell_{k}}{\left(\frac{1}{2} \sum_{i=1}^{k-1} \ell_{i}^{2}\right)+\frac{\sqrt{2}}{2} \ell_{k}},
$$

while the average taken at the ends of each block is

$$
\frac{1}{\# S \cap B_{r_{s}}} \sum_{(x, y) \in S \cap B_{r_{s}}} f(x, y)=\frac{\sqrt{2} \sum_{i=1}^{k} \ell_{i}}{\sum_{i=1}^{k} \ell_{i}^{2}} .
$$

The first average is larger than $1 / 2$, while the second tends to 0 ; each may be transferred to our measure-preserving system. This answers in the affirmative the question of whether there is a sequence of sets for which a subsequence converges while the larger sequence diverges.

This example illustrates the interaction between the metric of the group and the measure of the various blocks; the pointwise ergodic theorem fails for this example because we slice off a single face of the block at a time, and the growth requirement allows for these faces to outweigh the measure of the previous blocks.

There are multiple ways to rule out this kind of failure. For one, we can require our $\vec{a}_{k}$ to avoid the coordinate axes. Suppose for instance that we keep the same sequence of blocks $I_{k}$, but choose $\vec{a}_{k}$ along a diagonal:

$$
\vec{a}_{k}=\left(a_{k}, a_{k}, \ldots, a_{k}\right) .
$$

Proposition 2.9. With requirement (2.8), $\left\{S \cap B_{r}: r \geq 1\right\}$ is a pointwise $L^{1}$-good sequence of sets for any aperiodic $\mathbb{Z}^{d}$-action.

Proof. As in Bellow and Losert's proof for the block sequence, we will rely on the Tempelman Ergodic Theorem to complete the proof. The first three requirements are filled - the sets are all of finite volume, each is nested within the next, and the Følner property is satisfied through the lacunary growth of the diameters $\ell_{k}$. All that remains is the difference requirement.

Here we divide our set $S \cap B_{r}$ into two: a $S_{m}=\bigcup_{k=1}^{m}\left(\vec{a}_{k}+I_{k}\right)$, including everything up to the last complete prism in $S$, and a remainder $R$, consisting of 
everything else. The crucial difference between this and the earlier example is that the remainder $R$ must be either trivial or properly $d$-dimensional.

Since

$$
S \cap B_{r}=S_{m} \cup R
$$

we have

$$
\begin{aligned}
\#\left(S \cap B_{r}-S \cap B_{r}\right) & \leq \#\left(S_{m}-S_{m}\right)+\#\left(R-S_{m}\right)+\#\left(S_{m}-R\right)+\#(R-R) \\
& =\#\left(S_{m}-S_{m}\right)+2 \#\left(R-S_{m}\right)+\#(R-R) .
\end{aligned}
$$

We will deal with the first term first, noting that this would complete the proof of our claim were we only interested in the subsequence consisting of sets with only complete prisms.

Since $S_{m}=\left(\vec{a}_{m}+I_{m}\right) \cup S_{m-1}$, we see that

$$
\begin{aligned}
\#\left(S_{m}-S_{m}\right) \leq & \#\left\{\left(\vec{a}_{m}+I_{m}\right)-\left(\vec{a}_{m}+I_{m}\right)\right\}+2 \#\left\{\left(\vec{a}_{m}+I_{m}\right)-S_{m-1}\right\} \\
& +\#\left\{S_{m-1}-S_{m-1}\right\} .
\end{aligned}
$$

For the first term, we have

$$
\#\left\{\left(\vec{a}_{m}+I_{m}\right)-\left(\vec{a}_{m}+I_{m}\right)\right\}=\#\left(I_{m}-I_{m}\right) \leq \#\left(B_{\ell_{m}}-B_{\ell_{m}}\right) \leq \# B_{2 \ell_{m}} \leq C \# I_{m} .
$$

For the third term, we have that $S_{m-1} \subset B_{\left|\vec{a}_{m-1}\right|+\ell_{m-1}}$, thus the difference

$$
S_{m-1}-S_{m-1} \subset B_{\left|\vec{a}_{m-1}\right|+\ell_{m-1}}-B_{\left|\vec{a}_{m-1}\right|+\ell_{m-1}},
$$

which, in turn, is contained in $B_{\left|\vec{a}_{m-1}\right|+\ell_{m-1}}$. The volume of this ball is a constant multiple of $\left(\left|\vec{a}_{m-1}\right|+\ell_{m-1}\right)^{d}$. But by our second condition on the spacing sequence above,

$$
\left(\left|\vec{a}_{m-1}\right|+\ell_{m-1}\right)^{d} \leq\left(\frac{1}{C} \ell_{m}+\ell_{m-1}\right)^{d},
$$

whence we have that this difference is less than $C^{d} \ell_{m}^{d}$ for some constant $C$.

For the second difference, we note that shifting a set in $\mathbb{Z}^{d}$ does not change its measure, and that $S_{m-1} \subset B_{\left|\vec{a}_{m-1}\right|+\ell_{m-1}}$. So

$$
\begin{aligned}
\#\left\{\left(\vec{a}_{m}+I_{m}\right)-S_{m-1}\right\} & =\#\left\{\vec{a}_{m}+I_{m}-S_{m-1}\right\}=\#\left\{I_{m}-S_{m-1}\right\} \\
& <\#\left\{B_{\ell_{m}}-B_{\left|\vec{a}_{m-1}\right|+\ell_{m-1}}\right\} \leq \# B_{\left|\vec{a}_{m-1}\right|+\ell_{m-1}+\ell_{m}},
\end{aligned}
$$

which, as before, has a volume less than $C^{d} \ell_{m}^{d}$ for some constant $C$.

So we have that the difference \# $\left(S_{m}-S_{m}\right) \leq C \# S_{m}$.

The size of the second and third terms in (2.9) are dependent upon the diameter of $R$. Defining $s$ to be the diameter of $R$, we note that $s \leq \ell_{m+1}$ and the measure of $\# R \geq C s^{d}$, by our orignial conditions on the block $I_{m+1}$ and requirement (2.8).

For the third term, we note that

$$
R-R \subseteq\left(\vec{a}_{m+1}+B_{s}\right)-\left(\vec{a}_{m+1}+B_{s}\right) .
$$

Thus we have that

$$
\begin{aligned}
\#(R-R) & \leq \#\left(B_{s}-B_{s}\right) \\
& =\# B_{2 s} \\
& \leq C s^{d} \leq c \# R,
\end{aligned}
$$

where $C$ and $c$ are different constants. 
This leaves us with only the second term. Here we have

$$
\begin{aligned}
\#\left(R-S_{m}\right) & \leq \#\left(B_{s}-B_{c \ell_{m}}\right) \\
& \leq C \max \left(s^{d},\left(c \ell_{m}\right)^{d}\right) \leq C \#\left(S \cap B_{r}\right)
\end{aligned}
$$

for constants $c$ and $C$.

Remark. Taking another approach, we might instead place an additional requirement on the diameters, $\ell_{k}$, relative to the spacing vectors, $\vec{a}_{k}$, in order to ensure that the first set which might outweigh the previous blocks would necessarily be $d$-dimensional.

\section{Multivariable RAndom aVERages}

There are two natural ways that we might extend the results of [15] to $\mathbb{Z}^{d_{-}}$ actions, depending on the choice of random variables corresponding to $\vec{n}$. The first, which hews to the product structure of $\mathbb{Z}^{d}$, is to write $\xi_{\vec{n}}=\prod_{i=1}^{d} \xi_{i, n_{i}}$, where $\left\{\xi_{i, n}: 1 \leq i \leq d ; n \in \mathbb{N}\right\}$ are independent random variables. The other is to simply take independent random variables $\left\{\xi_{\vec{n}}: \vec{n} \in \mathbb{Z}^{d}\right\}$.

These different approaches can be characterized as the "plaid" and "speckled" approaches, respectively, according to the patterns of points that they select in $\mathbb{Z}^{2}$. The relevant distinction, for us, will concern the difference set: if we let $S_{N}^{\omega}$ denote the set of all $\vec{n}$ with $\xi_{\vec{n}}=1$ and $|\vec{n}| \leq N$, then in the speckled case, for any $\vec{k} \neq 0$ there will (with overwhelming probability) not be too many ways to write $\vec{k}$ as the difference of two elements of $S_{N}^{\omega}$ (and this number of representations will in fact be very close to its probabilistic mean); but in the plaid case, there will be significantly more representations whenever any of the components of $\vec{k}$ are zero. For this reason, we will first prove the result in the simpler speckled case.

Remark. We may also consider the "plaid diagonal" pattern obtained by taking $\xi_{i, n}=\xi_{1, n}$ for all $i$; however, this does not exhibit any behavior different from the first plaid case, and is more difficult to calculate.

Remark. One might hope that the product theory might help us to prove the maximal inequality for the plaid case directly from the one-dimensional result; however, this is not the case in $L^{1}$, any more than it is for the Bellow-Losert construction.

Remark. Our reliance on the "uniformity" of the ways to represent points as elements of $S_{N}^{\omega}-S_{N}^{\omega}$ gives us a natural bound on the maximal sparsity for which this technique could possibly work on random sets. Namely, the average number of representations of a point $\vec{k}$ with $|\vec{k}| \lesssim N$ should tend to infinity as $N \rightarrow \infty$, and thus we will need $\# S_{N}^{\omega} \gg \sqrt{\# B(0, N)} \approx N^{d / 2}$. Indeed, we will have a result precisely when our random sets have $\# S_{N}^{\omega} \approx N^{\alpha}$ with $\alpha>d / 2$.

3.1. Speckled random averages on $\mathbb{Z}^{d}$. Using the Fourier transform, it is an immediate extension of the one-dimensional theory that both the plaid and the speckled sequences are universally $L^{2}$-good.

Thus we need only prove a weak maximal inequality on $L^{1}$, and (by the positivity of the averaging operators) this only on dyadically increasing sets. Finally, by the Calderón transference principle (Lemma 5.7), we can instead prove this maximal 
theorem for the corresponding convolution operators on $\mathbb{Z}^{d}$. That is, we let $\left\{\xi_{\vec{n}}: \vec{n} \in\right.$ $\left.\mathbb{Z}^{d}\right\}$ be independent $\{0,1\}$-valued random variables, such that if $2^{j} \leq|\vec{n}|<2^{j+1}$, then $\mathbb{P}\left(\xi_{\vec{n}}=1\right)=2^{-\gamma j} \approx|\vec{n}|^{-\gamma}$. Then define

$$
\begin{aligned}
\mu_{j}^{(\omega)}(\vec{n}) & := \begin{cases}2^{(\gamma-d) j} \xi_{\vec{n}}(\omega), & 2^{j} \leq|\vec{n}|<2^{j+1} \\
0 & \text { otherwise },\end{cases} \\
\nu_{j}^{(\omega)}(\vec{n}) & := \begin{cases}\mu_{j}^{(\omega)}(\vec{n})-2^{-d j}, & 2^{j} \leq|\vec{n}|<2^{j+1} \\
0 & \text { otherwise. }\end{cases}
\end{aligned}
$$

Then the random convolution operators $f \rightarrow f * \mu_{j}^{(\omega)}$ correspond to our random ergodic averages, and the $\nu_{j}^{(\omega)}$ are mean 0 variants of the same. The role of the difference set, alluded to previously, is expressed in terms of the convolution of the mean 0 measure $\nu_{j}^{(\omega)}$ with its reflection $\tilde{\nu}_{j}^{(\omega)}(\vec{v})=\nu_{j}^{(\omega)}(-\vec{v})$, which we will use later in the argument. Clearly $\nu_{j}^{(\omega)} * \tilde{\nu}_{j}^{(\omega)}(0)=\left\|\nu_{j}^{(\omega)}\right\|_{\ell^{2}\left(\mathbb{Z}^{d}\right)}^{2}$, but at all nonzero points the convolution should be small:

Lemma 3.1. Let $\epsilon>0$. With probability 1 in $\Omega$, there exists $C_{\omega, \epsilon}<\infty$ such that

$$
\left\|\nu_{j}^{(\omega)} * \tilde{\nu}_{j}^{(\omega)}\right\|_{\ell^{\infty}\left(\mathbb{Z}_{\times}^{d}\right)} \leq C_{\omega, \epsilon} 2^{\left(\gamma-\frac{3 d}{2}+\epsilon\right) j},
$$

where $\mathbb{Z}_{\times}^{d}:=\mathbb{Z}^{d} \backslash\{0\}$. Furthermore,

$$
\left|\nu_{j}^{(\omega)} * \tilde{\nu}_{j}^{(\omega)}(0)\right| \leq C_{\omega, \epsilon} 2^{(\gamma-d) j} .
$$

This follows from the more general Lemma 5.11 and the Borel-Cantelli Lemma. We must now prove the following:

Theorem 3.2. For all $\gamma<d / 2$, there is a set of probability 1 in $\Omega$ such that for each $\omega$ in this set, there exists $C_{\omega}<\infty$ such that for every $\lambda>0$,

$$
\#\left\{\vec{n}: \sup _{j}\left|f * \mu_{j}^{(\omega)}(\vec{n})\right|>\lambda\right\} \leq \frac{C_{\omega}}{\lambda}\|f\|_{1} .
$$

This immediately implies Theorem 1.1, which gives multivariable averaging methods over sets of Banach density 0.

(We will suppress the superscripts $(\omega)$ in the rest of this section, and presume that $\nu_{j}$ are measures which satisfy the bounds in Lemma 3.1 for a constant $C$.)

Proof. The method of this proof begins with Calderón-Zygmund theory. We decompose our function $f$ in the standard fashion, and imitate the technique of Christ 8 for operators on $\mathbb{R}^{d}$ whose kernels are not properly differentiable. For the crucial parts, $B$, of our decomposition of $f$, we consider the inner products $\left\langle\nu_{j} * B, \nu_{j} * B\right\rangle=\left\langle\tilde{\nu}_{j} * \nu_{j} * B, B\right\rangle$ and use the extra cancellation of that convolution to obtain an especially strong $L^{2}$ bound. This is necessary in order to compensate for the waste of using an $L^{2}$ bound to prove an $L^{1}$ bound. This technique was first applied to pointwise ergodic theorems in $L^{1}$ by Urban and Zienkiewicz [23].

By scaling, we may assume that $\lambda \approx 1$. We will choose $\lambda$ to be a bit larger than 1 , but our choice will depend only on $d$ and $\omega$, not on $f$ or on $j$. We apply the standard discrete Calderón-Zygmund decomposition at height 1 , giving us $f=$ $g+b=g+\sum_{s, k} b_{s, k}$, where $\|g\|_{\infty} \leq 1$, each $b_{s, k}$ is supported on a discrete dyadic cube $Q_{s, k}$ with side length $2^{s},\left\|b_{s, k}\right\|_{1} \leq 2^{d s}$, and $\sum_{s, k}\left|Q_{s, k}\right| \leq 2^{d}\|f\|_{1}$. We can also require each $b_{s, k}$ to have mean 0 , but we shall not need this. 
Now we will use to our advantage several key properties of $\mu_{j}$ and $\nu_{j}$ to reduce this problem to its final form. First, we have normalized $\mu_{j}$ so that with probability 1 in $\Omega$, there is a $C_{\omega}$ such that $\left\|\mu_{j}\right\|_{1} \leq C_{\omega}$ for all $j$, and thus $\left\|g * \mu_{j}\right\|_{\infty} \leq C_{\omega}$ for all $j$. Therefore, if $\sup _{j}\left|f * \mu_{j}(\vec{n})\right|>\lambda$, then $\sup _{j}\left|\sum_{s, k} b_{s, k} * \mu_{j}(\vec{n})\right|>\lambda-C_{\omega}$.

We next split $b_{s}:=\sum_{k} b_{s, k}$ into two pieces, depending on its size. Define

$$
\begin{aligned}
b_{s}^{(j)}(\vec{n}) & := \begin{cases}b_{s}(\vec{n}), & \left|b_{s}(\vec{n})\right|>2^{(d-\gamma) j}, \\
0 & \text { otherwise, }\end{cases} \\
B_{s}^{(j)} & :=b_{s}-b_{s}^{(j)} .
\end{aligned}
$$

Note that the threshold $2^{(d-\gamma) j}$ is proportional to $\left|\operatorname{supp} \mu_{j}\right|$. This allows us to prove a bound on the support of $\sup _{j}\left|\sum_{s} b_{s}^{(j)} * \mu_{j}(\vec{n})\right|$, since $b_{s, k}$ cannot be large on a large set. In particular, if we let $b^{(j)}:=\sum_{s} b_{s}^{(j)}$,

$$
\begin{aligned}
& \#\left\{\vec{n}: \sup _{j}\left|\sum_{s} b_{s}^{(j)} * \mu_{j}(\vec{n})\right|>0\right\} \\
& \leq \sum_{j} \#\left\{\vec{n}:\left|b^{(j)} * \mu_{j}(\vec{n})\right|>0\right\} \\
& \leq \sum_{j}\left|\operatorname{supp} \mu_{j}\right| \cdot \#\left\{\vec{n}:|b(\vec{n})|>2^{(d-\gamma) j}\right\} \\
& \leq \sum_{j} C_{\omega} 2^{(d-\gamma) j} \sum_{i \geq j} \#\left\{\vec{n}: 2^{(d-\gamma) i}<|b(\vec{n})| \leq 2^{(d-\gamma)(i+1)}\right\} \\
& =C_{\omega} \sum_{i} \#\left\{\vec{n}: 2^{(d-\gamma) i}<|b(\vec{n})| \leq 2^{(d-\gamma)(i+1)}\right\} \sum_{j \leq i} 2^{(d-\gamma) j} \\
& \leq C_{\omega} \sum_{i} \#\left\{\vec{n}: 2^{(d-\gamma) i}<|b(\vec{n})| \leq 2^{(d-\gamma)(i+1)}\right\} \cdot C 2^{(d-\gamma) i} \\
& \leq C_{\omega}^{\prime}\|b\|_{1} \leq C_{\omega}^{\prime \prime}\|f\|_{1}
\end{aligned}
$$

because the second-to-last line is a lower sum for $b$. This is an acceptable bound, so we need only consider the contribution of $B_{s}^{(j)}$, which have the property $\left\|B_{s}^{(j)}\right\|_{\infty} \leq$ $2^{(d-\gamma) j}$. This will be precisely what we need to control the contribution of $\nu_{j} * \tilde{\nu}_{j}(0)$.

Since $\mu_{j}-\nu_{j}$ is simply an appropriately normalized average over all points of magnitude between $2^{j}$ and $2^{j+1}$, the weak $(1,1)$ bound on the standard maximal function tells us that

$$
\begin{aligned}
\#\left\{\vec{n}: \sup _{j}\left|\sum_{s} B_{s}^{(j)} *\left(\mu_{j}-\nu_{j}\right)(\vec{n})\right|>1\right\} & \leq \#\left\{\vec{n}: \sup _{j}|b| *\left|\mu_{j}-\nu_{j}\right|(\vec{n})>1\right\} \\
& \leq C\|b\|_{1} \leq C^{\prime}\|f\|_{1},
\end{aligned}
$$

and we may indeed replace $\mu_{j}$ with $\nu_{j}$.

Finally, if we let $Q_{s, k}^{*}$ denote the points that are within distance $2^{s}$ of the cube $Q_{s, k}$, then $b_{s, k} * \nu_{j}$ is supported in $Q_{s, k}^{*}$ if $j<s$. We take the exceptional set $E$ to be the union of all $Q_{s, k}^{*}$, and note that $|E| \leq C_{d}\|f\|_{1}$. Since $E$ is of acceptable size and supp $B_{s}^{(j)} * \nu_{j} \subset E$ for all $s>j$, we only need to bound the set

$$
\#\left\{\vec{n}: \sup _{j}\left|\sum_{s \leq j} B_{s}^{(j)} * \nu_{j}(\vec{n})\right|>1\right\} .
$$

This we will do using an $L^{2}$ bound. 
Remark. Most of the above reductions are standard, with the exception of splitting $b_{s, k}=b_{s, k}^{(j)}+B_{s, k}^{(j)}$ by height. This is done because $\nu_{j} * \tilde{\nu}_{j}(0)$ has no cancellation, and thus we must treat the contribution of a single delta mass in our inner product separately. The condition $\left\|B_{s, k}^{(j)}\right\|_{\infty} \leq 2^{(d-\gamma) j}$ precisely suffices to balance the trivial bound (3.4) in these terms. Note that there is no equivalent for this in the original problems on $\mathbb{R}^{d}$, since there the convolutions have a singularity rather than a delta mass at the origin; because of this, the analogous endpoint theorems require $f$ in the Hardy space $H^{1}$ rather than $L^{1}$.

By Chebyshev's Inequality,

$$
\begin{aligned}
\#\left\{\vec{n}: \sup _{j}\left|\sum_{s \leq j} B_{s}^{(j)} * \nu_{j}(\vec{n})\right|>1\right\} & \leq\left\|\sup _{j} \mid \sum_{s \leq j} B_{s}^{(j)} * \nu_{j}\right\| \|_{2}^{2} \\
& \leq \sum_{j}\left\|\sum_{s \leq j} B_{s}^{(j)} * \nu_{j}\right\|_{2}^{2} \\
& \leq 2 \sum_{j} \sum_{\substack{s, t: \\
s \leq t \leq j}}\left\langle B_{s}^{(j)} * \nu_{j}, B_{t}^{(j)} * \nu_{j}\right\rangle \\
& =2 \sum_{j} \sum_{\substack{s, t: \\
s \leq t \leq j}}\left\langle B_{s}^{(j)} * \nu_{j} * \tilde{\nu}_{j}, B_{t}^{(j)}\right\rangle .
\end{aligned}
$$

First we assume that supp $B_{s}^{(j)} \subset Q_{j, k_{1}}$ and supp $B_{t}^{(j)} \subset Q_{j, k_{2}}$, where each of these is a single dyadic cube of size $2^{j}$. Then it is easy to see that $\left\|B_{s}^{(j)}\right\|_{1} \leq 2^{d j}$, and thus by Lemma 3.1 .

$$
\begin{aligned}
\left|\left\langle B_{s}^{(j)} * \nu_{j} * \tilde{\nu}_{j}, B_{t}^{(j)}\right\rangle\right| \leq & \left\|\nu_{j} * \tilde{\nu}_{j}\right\|_{\ell^{\infty}\left(\mathbb{Z}_{\times}^{d}\right)}\left\|B_{s}^{(j)}\right\|_{1}\left\|B_{t}^{(j)}\right\|_{1}+\nu_{j} * \tilde{\nu}_{j}(0)\left\langle B_{s}^{(j)}, B_{t}^{(j)}\right\rangle \\
\leq & C_{\omega, \epsilon} 2^{(\gamma-3 d / 2+\epsilon) j}\left\|B_{s}^{(j)}\right\|_{1}\left\|B_{t}^{(j)}\right\|_{1} \\
& +C_{\omega, \epsilon} 2^{(\gamma-d) j} \delta_{s=t}\left\|B_{s}^{(j)}\right\|_{\infty}\left\|B_{t}^{(j)}\right\|_{1} \\
\leq & C_{\omega, \epsilon} 2^{(\gamma-d / 2+\epsilon) j}\left\|B_{t}^{(j)}\right\|_{1}+C_{\omega, \epsilon} \delta_{s=t}\left\|B_{t}^{(j)}\right\|_{1} .
\end{aligned}
$$

We can remove the restriction on the supports since the inner product is 0 whenever the support of $B_{s}^{(j)}$ and the support of $B_{t}^{(j)}$ are separated by at least $2^{j+1}$; thus the double sum over all $Q_{j, k_{1}}$ and $Q_{j, k_{2}}$ is, up to a fixed constant, a single sum. Therefore we have

$$
\begin{aligned}
\#\left\{\vec{n}: \sup _{j}\left|\sum_{s \leq j} B_{s}^{(j)} * \nu_{j}(\vec{n})\right|>1\right\} & \lesssim \sum_{j} \sum_{\substack{s, t: \\
s \leq t \leq j}} 2^{(\gamma-d / 2+\epsilon) j}\left\|B_{t}^{(j)}\right\|_{1}+\delta_{s=t}\left\|B_{t}^{(j)}\right\|_{1} \\
& \leq \sum_{j} \sum_{t}\left(j 2^{(\gamma-d / 2+\epsilon) j}+1\right)\left\|B_{t}^{(j)}\right\|_{1},
\end{aligned}
$$

and clearly, this is $\leq\|b\|_{1} \leq C\|f\|_{1}$ as long as $\gamma<d / 2-\epsilon$. Since $\epsilon>0$ is arbitrary, we have proved Theorem 3.2 
3.2. Plaid random averages on $\mathbb{Z}^{d}$. In this section, we take independent random variables $\left\{\xi_{i, n}: 1 \leq i \leq d ; n \in \mathbb{N}\right\}$ with $\mathbb{P}\left(\xi_{i, n}=1\right)=n^{-\alpha}$. We define

$$
\begin{aligned}
\mu_{j}^{(\omega)}(\vec{n}) & := \begin{cases}2^{d(\alpha-1) j} \prod_{i=1}^{d} \xi_{i, n_{i}}(\omega), & 2^{j} \leq|\vec{n}|<2^{j+1}, n_{i}>0, \text { for all } i, \\
0 & \text { otherwise },\end{cases} \\
\nu_{j}^{(\omega)}(\vec{n}) & := \begin{cases}\mu_{j}^{(\omega)}(\vec{n})-\prod_{i=1}^{d} n_{i}^{-\alpha}, & 2^{j} \leq|\vec{n}|<2^{j+1}, n_{i}>0, \text { for all } i, \\
0 & \text { otherwise. }\end{cases}
\end{aligned}
$$

As mentioned before, our decomposition of the random measure $\nu_{j}^{(\omega)} * \tilde{\nu}_{j}^{(\omega)}$ will be more complicated because the additional structure removes part of the cancellation at points where some of the coordinates are zero.

Lemma 3.3. Let $\epsilon>0$. With probability 1 in $\Omega$, there exists $C_{\omega, \epsilon}<\infty$ such that

$$
\nu_{j}^{(\omega)} * \tilde{\nu}_{j}^{(\omega)}=\sum_{I \subset\{1, \ldots, d\}} \chi_{j, I}^{(\omega)},
$$

where each $\chi_{j, I}^{(\omega)}$ is supported on $\left\{\vec{n}: n_{i} \neq 0\right.$ for all $\left.i \in I\right\}$, and for all $I \neq \emptyset$,

$$
\left\|\chi_{j, I}^{(\omega)}\right\|_{\infty} \leq C_{\omega, \epsilon} 2^{\left(-d-\frac{|I|}{2}+d \alpha+\epsilon\right) j} .
$$

Furthermore,

$$
\left|\nu_{j}^{(\omega)} * \tilde{\nu}_{j}^{(\omega)}(0)\right| \leq C_{\omega, \epsilon} 2^{d(\alpha-1) j}
$$

Remark. We could aggregate all of the $\chi_{j, I}^{(\omega)}$ with $I \neq \emptyset$ into a single function, and for $\alpha<\frac{1}{2 d}$ the overall $\ell^{\infty}$ bound would be good enough to prove the weak maximal inequality just as we proved Theorem 3.2 . However, with a more targeted Calderón-Zygmund decomposition, we can do better, and obtain the weak maximal inequality for $\alpha<1 / 2$. Thus we can prove an $L^{1}$ pointwise ergodic theorem for plaid sets which are just as sparse as the speckled sets we have proved it for.

Theorem 3.4. For all $\alpha<1 / 2$, there is a set of probability 1 in $\Omega$ such that for each $\omega$ in this set, there exists $C_{\omega}<\infty$ such that for every $\lambda>0$,

$$
\#\left\{\vec{n}: \sup _{j}\left|f * \mu_{j}^{(\omega)}(\vec{n})\right|>\lambda\right\} \leq \frac{C_{\omega}}{\lambda}\|f\|_{1} .
$$

Corollary 3.5. For all $\alpha<1 / 2$, there is a set of probability 1 in $\Omega$ such that every $\omega$ in this set has the following property: Let $\left\{\vec{a}_{k}: k \in \mathbb{N}\right\}$ be an enumeration of the set $\left\{\vec{n}: \xi_{i, n_{i}}(\omega)=1\right.$ for all $\left.i\right\}$ with $\left|\vec{a}_{k}\right|$ increasing. Then for any measure-preserving $\mathbb{Z}^{d}$-action $\mathcal{T}$ and any $f \in L^{1}(X)$, the averages

$$
A_{N} f(x):=\frac{1}{N} \sum_{k=1}^{N} f \circ \mathcal{T}\left(\vec{a}_{k}\right)(x)
$$

converge a.e. in $X$.

Proof. The proof of Theorem 3.4 follows the same lines as that of Theorem 3.2 , with one substitution: we will have to do more when we split $b_{s, k}=b_{s, k}^{(j)}+B_{s, k}^{(j)}$, so that we can get better bounds on the pieces of the inner product which correspond to the various $\chi_{j, I}$.

Let us jump ahead to that inner product to determine the bounds we will need; again we will assume that each of $B_{s}^{(j)}$ and $B_{t}^{(j)}$ is supported on a dyadic cube 
of side length $2^{j}$. Let $\vec{n}_{I} \in \mathbb{Z}^{\# I}$ denote the projection of $\vec{n}$ onto the coordinates indexed by $I$, and $\vec{n}_{I}^{\perp} \in \mathbb{Z}^{d-\# I}$ the projection onto the other $d-\# I$ coordinates. Then

$$
\begin{aligned}
\left|\left\langle B_{s}^{(j)} * \chi_{j, I}, B_{t}^{(j)}\right\rangle\right| & =\left|\sum_{\vec{n}} \sum_{\vec{m}} \chi_{j, I}(\vec{n}-\vec{m}) B_{s}^{(j)}(\vec{m}) \bar{B}_{t}^{(j)}(\vec{n})\right| \\
& =\left|\sum_{\vec{n}} \bar{B}_{t}^{(j)}(\vec{n}) \sum_{\vec{m}_{I}} \chi_{j, I}\left(n_{I}-m_{I}, 0\right) B_{s}^{(j)}\left(\vec{m}_{I}, \vec{n}_{I}^{\perp}\right)\right| \\
& \leq \sum_{\vec{n}}\left|\bar{B}_{t}^{(j)}(\vec{n})\right| \cdot\left\|\chi_{I}\right\|_{\infty} \sum_{\vec{m}_{I}}\left|B_{s}^{(j)}\left(\vec{m}_{I}, \vec{n}_{I}^{\perp}\right)\right| \\
& \leq\left\|B_{t}^{(j)}\right\|_{1}\left\|\chi_{j, I}\right\|_{\infty}\left(\sup _{\vec{n}_{I}^{\perp}} \sum_{\vec{n}_{I}}\left|B_{s}^{(j)}\left(\vec{n}_{I}, \vec{n}_{I}^{\perp}\right)\right|\right)
\end{aligned}
$$

Just as the delta mass at 0 for the speckled averages made it useful to define $B_{s}^{(j)}$ so that its $\ell^{\infty}$ norm was suitably bounded, in the plaid case we will want to define $B_{s}^{(j)}$ so that we have bounds on the collection of $\ell^{\infty}\left(\ell^{1}\right)$ mixed norms that appear in the last line above. As in the speckled case, the bounds we can obtain from properties of the support will suffice.

That is, instead of (3.7), we let $Q_{j, \vec{n}}$ denote the dyadic cube with side length $2^{j}$ containing $\vec{n}$, and define for $1 \leq \# I<d$

$$
b_{s}^{j, I}(\vec{n}):= \begin{cases}b_{s}(\vec{n}), & \sum_{\left\{\vec{m}_{I}:\left(\vec{m}_{I}, \vec{n}_{I}^{\perp}\right) \in Q_{j, \vec{n}}\right\}}\left|b_{s}\left(\vec{m}_{I}, \vec{n}_{I}^{\perp}\right)\right|>2^{(d-\alpha(d-\# I)+\epsilon) j} \\ 0 & \text { otherwise. }\end{cases}
$$

For $I=\emptyset$, define as before

$$
b_{s}^{j, \emptyset}(\vec{n}):= \begin{cases}b_{s}(\vec{n}), & \left|b_{s}(\vec{n})\right|>2^{(d-d \alpha) j} \\ 0 & \text { otherwise. }\end{cases}
$$

Note that this bound lacks the factor of $2^{\epsilon j}$; this exact bound is crucial, since for this term we will have no extra cancellation in $\chi_{j, I}$ for $\alpha$ small.

Although we would have problems with double-counting if we simply subtracted these pieces from the original $b_{s}$, it is clear that we can define $B_{s}^{(j)}$ such that

$$
\sum_{\vec{m}_{I}:\left(\vec{m}_{I}, \vec{n}_{I}^{\perp}\right) \in Q_{j, \vec{n}}}\left|B_{s}^{(j)}\left(\vec{m}_{I}, \vec{n}_{I}^{\perp}\right)\right| \leq 2^{(d-\alpha(d-\# I)+\epsilon) j}
$$

and

$$
\left|B_{s}^{(j)}-b_{s}\right| \leq \sum_{I \subsetneq\{1, \ldots, d\}}\left|b_{s}^{j, I}\right| .
$$

Thus we have split up $b_{s}$ with regard to the size of the relevant mixed norm on the cubes $Q_{j, \vec{n}}$. The contribution of the terms $b_{s}^{j, \emptyset}$ is bounded as before. For the others, we will use the geometry of the supports and the fact that we can spare an extra $2^{\epsilon j}$.

If we let $V_{j, I}:=\left\{\left(\vec{v}_{I}, 0\right):\left|\vec{v}_{I}\right|<2^{j+1}\right\}$, then we note that supp $\mu_{j}$ can be covered by $\leq C_{\omega} 2^{(d-\# I)(1-\alpha) j}$ copies of $V_{j, I}$, that $\operatorname{supp} b_{s}^{j, I}$ can be covered by 
$\leq 2^{-(d-\alpha(d-\# I)+\epsilon) j}\left\|b_{s}\right\|_{1}$ copies of $V_{j, I}$, and that $\left|V_{j, I}+V_{j, I}\right| \leq 2^{\# I(j+2)}$. Therefore

$$
\begin{aligned}
\#\{\vec{n} & \left.: \sup _{j}\left|\sum_{s} b_{s}^{j, I} * \mu_{j}(\vec{n})\right|>0\right\} \\
& \leq \sum_{j} \sum_{s}\left|\left(\operatorname{supp} b_{s}^{j, I}\right)+\left(\operatorname{supp} \mu_{j}\right)\right| \\
& \lesssim \sum_{j} \sum_{s} 2^{\# I(j+2)} 2^{-(d-\alpha(d-\# I)+\epsilon) j}\left\|b_{s}\right\|_{1} 2^{(d-\# I)(1-\alpha) j} \\
& \leq\|b\|_{1} \sum_{j} 2^{-\epsilon j} \lesssim\|f\|_{1} .
\end{aligned}
$$

Therefore we may reduce as before to considering the contribution of $B_{s}^{(j)}$. The argument is again identical to the proof of Theorem 3.2 until the point where the inner product is calculated. For $I \neq \emptyset$ we have (again under the assumption, easily removed, that $B_{s}^{(j)}$ and $B_{t}^{(j)}$ are each supported on single dyadic cubes of size $2^{j}$ ) that

$$
\begin{aligned}
\left|\left\langle B_{s}^{(j)} * \nu_{j} * \tilde{\nu}_{j}, B_{t}^{(j)}\right\rangle\right| & \leq \sum_{I \subset\{1, \ldots, d\}}\left|\left\langle B_{s}^{(j)} * \chi_{j, I}, B_{t}^{(j)}\right\rangle\right| \\
& \leq\left\|B_{t}^{(j)}\right\|_{1}\left\|\chi_{j, I}\right\|_{\infty}\left(\sup _{\vec{n}_{I}^{\perp}} \sum_{\vec{n}_{I}}\left|B_{s}^{(j)}\left(\vec{n}_{I}, \vec{n}_{I}^{\perp}\right)\right|\right) \\
& \lesssim\left\|B_{t}^{(j)}\right\|_{1} 2^{\left(-d-\frac{\# I}{2}+d \alpha+\epsilon\right) j} 2^{(d-\alpha(d-\# I)+\epsilon) j} \\
& \leq\left\|B_{t}^{(j)}\right\|_{1} 2^{\left(\left(\frac{1}{2}-\alpha\right) \# I+2 \epsilon\right) j}
\end{aligned}
$$

and so the terms are bounded for $\epsilon$ sufficiently small, as long as $\alpha<\frac{1}{2}$.

\section{Sparse ARITHMETIC SETS IN $\mathbb{Z}^{d}$}

In the following two sections, we seek to adapt a recent construction by M. Christ [10] to a higher-dimensional setting. That construction takes polynomial subsets of finite groups $\mathbb{Z}_{p}^{m}$ and transfers them to $\mathbb{Z}^{d}$ via Freiman isomorphisms. Weil's inequality on complete character sums implies that the uniform probability measure on such a set will be optimally pseudo-random (in the sense of its Fourier transform), which suffices to prove a weak $(1,1)$ bound on these averages.

As in Section 3 , we have our choice between extending this one-dimensional result into a product set (plaid) construction or a natively $d$-dimensional (speckled) version. (One of the means by which we can vary the sparse sequence's rate of growth, in fact, is to project an $m$-dimensional set down to $\mathbb{Z}^{d}$, for $m$ a multiple of d.) The proofs will closely follow those in [10]; in the speckled case, we will take an isomorphism from $\mathbb{Z}_{p}^{m}$ to $\mathbb{Z}^{d}$ rather than $\mathbb{Z}$, while in the plaid case we observe that the relevant Fourier estimates factorize into a product of the estimates for the original sequence.

In addition, we prove an oscillational inequality for these averages, which implies a pointwise ergodic theorem; the corresponding result was not included in [10]. 
4.1. A speckled construction. Let $c$ and $C$ be constants larger than 1 and suppose that $\left\{p_{k}\right\}$ is a sequence of primes satisfying

$$
c p_{k} \leq p_{k+1}<C p_{k}
$$

for all $k$. (Later, we will want $p_{k} \approx 2^{\gamma k}$ for some $\gamma>0$, but this can be achieved by just repeating terms as necessary.)

As mentioned before, we will construct $m$-dimensional sparse sets, where $m=q d$ for some positive integer $q$; the case $q=1$ gives us a "native" $d$-dimensional set, while higher values of $q$ give us sparser sets. So we fix a positive integer $q$ and choose a sequence of vectors $\left\{\vec{a}_{k}\right\} \subset \mathbb{Z}^{d}$ such that

$$
\left|\vec{a}_{k-1}\right|+p_{k-1}^{q}<\left|\vec{a}_{k}\right| \leq C p_{k}^{q} .
$$

Let $[n]$ denote $n \bmod p_{k}$, and define the sequence

$$
x_{k, j}=\left(\sum_{i=1}^{q} p^{i-1}\left[j^{i}\right]_{p_{k}}, \sum_{i=1}^{q} p^{i-1}\left[j^{q+i}\right]_{p_{k}}, \ldots, \sum_{i=1}^{q} p^{i-1}\left[j^{(d-1) q+i}\right]_{p_{k}}\right), 0 \leq j<p_{k},
$$

and the set

$$
S_{k}=\left\{\vec{a}_{k}+x_{k, j}: 0 \leq j<p_{k}\right\} .
$$

This is the image of the set $\left\{\left(j, j^{2}, \ldots, j^{m}\right): 0 \leq j<p_{k}\right\} \subset \mathbb{Z}_{p_{k}}^{m}$ under a suitable Freiman isomorphism. The main reason we have chosen such a set is that it enjoys near-optimal Fourier bounds thanks to Weil's theorem on character sums:

Theorem 4.1 (Weil, [24]). Suppose $f \in \mathbb{Z}_{p}[x]$ is a polynomial and $p$ does not divide the degree, $m$, of $f$. Then

$$
\left|\sum_{n \in \mathbb{Z}_{p}} e(f(n) / p)\right| \leq(m-1) p^{1 / 2} .
$$

(In this section, we shall use $e(\theta)$ to denote the character $e^{2 \pi i \theta}$.) The size requirements on $\left\{\vec{a}_{k}\right\}$ ensure that the collection $\left\{S_{k}\right\}$ is disjoint; in fact, all the elements of $S_{k}$ lie in a shell of elements whose lengths are larger than $(C+1) p_{k}^{q}$ but less than $C p_{k+1}^{q}$. We also have that $\# S_{k}=p_{k}$.

Let $S=\bigcup_{k=1}^{\infty} S_{k}=\left\{x_{k, j}: k \in \mathbb{N}, 0 \leq j<p_{k}\right\}$. As a consequence of the sparseness of the individual sets $S_{k}$ and the lacunary nature of the sequence $\left\{p_{k}\right\}$, the sparsity of the set $S$ is similar to the sequence $\left(n^{q}, \ldots, n^{q}\right)$. We wish to prove the following $L^{1}$ pointwise ergodic theorem:

Theorem 4.2. Let $\left\{\vec{n}_{i}\right\}_{i \in \mathbb{N}}$ be an enumeration of $\left\{a_{k}+x_{k, j}\right\}$ in the dictionary ordering. Then for any measure-preserving $\mathbb{Z}^{d}$-action $\mathcal{T}$ and any $f \in L^{1}(X)$, the averages

$$
A_{N} f(x):=\frac{1}{N} \sum_{i=1}^{N} f \circ \mathcal{T}\left(\vec{n}_{i}\right)(x)
$$

converge almost everywhere in $X$.

As usual, this result will follow from an $L^{2}$ pointwise result and a weak $(1,1)$ maximal inequality. Using the standard transference arguments, it suffices to prove an oscillational inequality and a weak maximal inequality for the corresponding convolution operators on $\mathbb{Z}^{d}$. 
For the $L^{2}$ result, we will consider the averages on $\mathbb{Z}^{d}$,

$$
A_{N} f(\vec{v}):=\frac{1}{N} \sum_{i=1}^{N} f\left(v+n_{i}\right) .
$$

We must prove the following oscillational inequality, for any given lacunary $I \subset \mathbb{N}$ :

Theorem 4.3. For any sequence $t_{1} \leq t_{2} \leq \cdots$ with $t_{n} \in I$ for all $n$,

$$
\sum_{n}\left\|\sup _{t_{n-1} \leq t \leq t_{n}, t \in I}\left|A_{t} f-A_{t_{n}} f\right|\right\|_{2}^{2} \leq C\|f\|_{2}^{2} .
$$

We will prove this (in Section 4.1.2) by comparing $A_{t}$ to a more standard average, such that the Fourier norm of the difference is small. When transferred back to the dynamical system, such an oscillational inequality will directly imply an $L^{2}$ pointwise ergodic theorem (see for instance Jones, Kaufman, Rosenblatt and Wierdl [13]).

Then, since the blocks grow exponentially in size, we need only prove the $L^{1}$ weak maximal inequality for complete blocks:

Theorem 4.4. Let $S_{k}$ be defined as above. Then the maximal function

$$
M f(\vec{n})=\sup _{N} \frac{1}{\#\left(\bigcup_{k=1}^{N} S_{k}\right)} \sum_{\vec{m} \in \bigcup_{k=1}^{N} S_{k}}|f(\vec{n}+\vec{m})|
$$

satisfies a weak- $(1,1)$ inequality; that is, there is a constant $C$ so that for any $\lambda>0$ we have

$$
\#\{\vec{n}: M f(\vec{n})>\lambda\}<\frac{C}{\lambda}\|f\|_{\ell^{1}\left(\mathbb{Z}^{d}\right)} .
$$

4.1.1. Proof of Theorem 4.4. Again using a transference argument to reduce to a question about convolution operators on $\mathbb{Z}^{d}$, we seek to apply the following theorem from [10.

Theorem 4.5. Let $G$ be a discrete group and $\gamma>0$. Suppose the sequences of functions $\mu_{k}, \nu_{k}: G \rightarrow \mathbb{C}$ satisfy the following requirements:

(1) the maximal operator $\sup _{k}|f| *\left|\nu_{k}\right|$ is of weak type $(1,1)$ on $G$,

(2) for each $\mu_{k}$, \# $\left(\operatorname{supp}\left(\mu_{k}\right)\right) \leq C 2^{k \gamma}$ for some constant $C$, and

(3) $\left\|f *\left(\mu_{k}-\nu_{k}\right)\right\|_{\ell^{2}(G)} \leq C 2^{-k \gamma / 2}\|f\|_{\ell^{2}(G)}$ for all $f \in \ell^{2}(G)$.

Then the maximal operator $\sup _{k}\left|f * \mu_{k}\right|$ is of weak type $(1,1)$.

Henceforward we will suppress our $k$ subscripts and identify $\mathbb{Z}_{p}^{m}$ with its own dual group.

If $f$ is a function on $\mathbb{Z}_{p}^{m}$, then the Fourier transform of $f$ is

$$
\hat{f}(\vec{\xi})=\sum_{\vec{n} \in \mathbb{Z}_{p}^{m}} f(\vec{n}) e\left(\frac{\vec{n} \cdot \vec{\xi}}{p}\right) .
$$


We then have the inequalities

$$
\begin{aligned}
\|\widehat{f g}\|_{\ell^{\infty}} & \leq \frac{1}{p^{m}}\|\hat{f}\|_{\ell^{1}}\|\hat{g}\|_{\ell^{\infty}}, \text { and } \\
\|f * g\|_{\ell^{2}} & \leq \frac{1}{p^{m / 2}}\|\hat{f}\|_{\ell^{2}}\|\hat{g}\|_{\ell^{\infty}}=\|f\|_{\ell^{2}}\|\hat{g}\|_{\ell^{\infty}} .
\end{aligned}
$$

Finally, we will again make use of Weil's theorem for complete character sums (Theorem 4.1). Let $m=d q$. Our goal will be to produce measures $\mu^{\prime}$ and $\nu^{\prime}$ on $\mathbb{Z}_{p}^{m}$ that satisfy the analogues of the conditions in Theorem 4.5. We will then apply a linear operator to move from $\mathbb{Z}_{p}^{m}$ to $\mathbb{Z}^{m}$. A second operator will then transfer these measures to $\mathbb{Z}^{d}$.

Proof of Theorem 4.4. We begin by defining a probability measure on $\mathbb{Z}_{p}^{m}$ :

$$
\mu^{\prime}=\frac{1}{p} \sum_{j=0}^{p-1} \delta_{\left(j, j^{2}, \ldots, j^{m}\right)} .
$$

Note that

$$
\#\left(\operatorname{supp}\left(\mu^{\prime}\right)\right)=p .
$$

Thus $\mu^{\prime}$ satisfies the second requirement of Theorem 4.5 if $p_{k} \approx 2^{k \gamma}$.

We then define a second measure:

$$
\nu^{\prime}=\frac{1}{p^{m}} \sum_{\vec{j} \in \mathbb{Z}_{p}^{m}} \delta_{\vec{j}} .
$$

This will lead to a measure $\nu$ which satisfies the first requirement.

We are left with the third condition. By our inequality (4.6), we have that

$$
\left\|f *\left(\mu^{\prime}-\nu^{\prime}\right)\right\|_{\ell^{2}} \leq\|f\|_{\ell^{2}}\left\|\widehat{\mu^{\prime}-\nu^{\prime}}\right\|_{\ell^{\infty}} .
$$

It remains to prove an appropriate bound on $\left|\widehat{\mu^{\prime}-\nu^{\prime}}\right|$, using Theorem 4.1. The Fourier transform of $\mu^{\prime}$ is

$$
\begin{aligned}
\widehat{\mu^{\prime}}(\vec{\theta}) & =\sum_{\vec{n} \in \mathbb{Z}_{p}^{m}} \mu^{\prime}(\vec{n}) e\left(\frac{\vec{n} \cdot \vec{\theta}}{p}\right) \\
& =\frac{1}{p} \sum_{j=0}^{p-1} \sum_{\vec{n} \in \mathbb{Z}_{p}^{m}} \delta_{\left(j, j^{2}, \ldots, j^{m}\right)}(\vec{n}) e\left(\frac{\vec{n} \cdot \vec{\theta}}{p}\right) \\
& =\frac{1}{p} \sum_{j=0}^{p-1} e\left(\frac{j \theta_{1}+j^{2} \theta_{2}+\ldots+j^{m} \theta_{m}}{p}\right) .
\end{aligned}
$$

By the theorem, we then must have (for all $\vec{\theta} \neq 0$ )

$$
\widehat{\mu^{\prime}}(\vec{\theta}) \leq \frac{m-1}{p^{1 / 2}}
$$


Also, because

$$
\begin{aligned}
\widehat{\nu^{\prime}}(\vec{\theta}) & =\sum_{\vec{n} \in \mathbb{Z}_{p}^{m}} \frac{1}{p^{m}} \sum_{\vec{j} \in \mathbb{Z}_{p}^{m}} \delta_{\vec{j}}(\mathbf{n}) e\left(\frac{\vec{n} \cdot \vec{\theta}}{p}\right) \\
& = \begin{cases}1 & \text { if } \vec{\theta}=\overrightarrow{0}, \\
0 & \text { otherwise }\end{cases}
\end{aligned}
$$

and

$$
\widehat{\mu^{\prime}}(\overrightarrow{0})=1
$$

we have, for $\vec{\theta} \neq \overrightarrow{0}$, that

$$
\left|\widehat{\mu^{\prime}-\nu^{\prime}}\right| \leq \frac{m-1}{p^{1 / 2}}
$$

while for $\vec{\theta}=\overrightarrow{0}$, the difference is 0 .

Having found suitable measures $\mu^{\prime}$ and $\nu^{\prime}$ on $\mathbb{Z}_{p}^{m}$, we now construct measures on $\mathbb{Z}^{d}$ with the same Fourier properties. We will first create a "smooth cutoff" version on $\mathbb{Z}_{3 p}^{m}$ and then transfer this to $\mathbb{Z}^{d}$; since the result majorizes our desired measures $\mu$ and $\nu$, we may thus obtain the weak inequality for them.

First we will build a smoothing function, $\phi$. We identify $\mathbb{Z}_{3 p}^{m}$ with $[-p, 2 p-1]^{m}$ and $\mathbb{Z}_{3 p}$ with $[-p, 2 p-1]$. We define the function $\varphi: \mathbb{Z}_{3 p} \rightarrow \mathbb{R}$ so that

$$
\varphi(n)= \begin{cases}1 & \text { if } n \in[0, p-1] \\ 0 & \text { if } n \in\left[-p, \frac{-p-1}{2}\right] \cup\left[\frac{3}{2}(p-1), 2 p-1\right], \text { and } \\ \text { affine } & \text { otherwise. }\end{cases}
$$

We then define $\phi: \mathbb{Z}_{3 p}^{m} \rightarrow \mathbb{R}$ as a product of $\varphi$ 's:

$$
\phi(\vec{n})=\prod_{i=1}^{m} \varphi\left(n_{i}\right)
$$

We will also require the functions $\tau_{p}: \mathbb{Z}^{m} \rightarrow \mathbb{Z}_{p}^{m}$ and $\tau_{3 p}: \mathbb{Z}^{m} \rightarrow \mathbb{Z}_{3 p}^{m}$, given by

$$
\begin{aligned}
\tau_{p}(\vec{x}) & =\left(\left[x_{1}\right]_{p},\left[x_{2}\right]_{p}, \ldots,\left[x_{m}\right]_{p}\right), \\
\tau_{3 p}(\vec{x}) & =\left(\left[x_{1}\right]_{3 p},\left[x_{2}\right]_{3 p}, \ldots,\left[x_{m}\right]_{3 p}\right) .
\end{aligned}
$$

We can now transfer from measures on $\mathbb{Z}_{p}^{m}$ to "smoothly cut-off" measures on $\mathbb{Z}^{m}$. Define $\Gamma_{1}: \ell^{\infty}\left(\mathbb{Z}_{p}^{m}\right) \rightarrow \ell^{\infty}\left(\mathbb{Z}^{m}\right)$ by

$$
\Gamma_{1}(f)(\vec{j})=\mathbf{1}_{[-p, 2 p-1]^{m}}(\vec{j}) \phi\left(\tau_{3 p}(\vec{j})\right) f\left(\tau_{p}(\vec{j})\right) .
$$

Let $\vec{\theta} \in \mathbb{T}^{m}$ and write $\vec{\theta}=\vec{\xi} / 3 p+\vec{\eta}$ with $\vec{\xi} \in \mathbb{Z}^{m}$ and $\left|\eta_{i}\right| \leq C / p$ for all $1 \leq i \leq m$. The Fourier transform of $\Gamma_{1}(f)$ is

$$
\begin{aligned}
\widehat{\Gamma_{1}(f)}(\vec{\theta}) & =\sum_{\vec{j} \in \mathbb{Z}^{m}} \Gamma_{1}(f)(\vec{j}) e(\vec{j} \cdot \vec{\theta}) \\
& =\sum_{\vec{j} \in[-p, 2 p-1]^{m}} \phi(\vec{j}) f\left(\tau_{p}(\vec{j})\right) e(\vec{j} \cdot \vec{\eta}) e\left(\frac{\vec{j} \cdot \vec{\xi}}{3 p}\right) .
\end{aligned}
$$

Changing perspective, we may consider this as the Fourier transform on the group $\mathbb{Z}_{3 p}^{m}$ of the product of $f$ and $\psi(\vec{x})=\phi(\vec{x}) e(\vec{x} \cdot \vec{\eta})$. By inequality (4.5), then, 
we have

$$
\left\|\widehat{\Gamma_{1}(f)}(\vec{\theta})\right\|_{L^{\infty}\left(\mathbb{T}^{m}\right)} \leq \frac{1}{p^{m}}\|\hat{f}\|_{\ell^{\infty}\left(\mathbb{Z}_{3 p}^{m}\right)}\|\hat{\psi}\|_{\ell^{1}\left(\mathbb{Z}_{3 p}^{m}\right)}
$$

We plan to set

$$
\begin{aligned}
& \mu^{\prime \prime}=\Gamma_{1}\left(\mu^{\prime}\right) \text { and } \\
& \nu^{\prime \prime}=\Gamma_{1}\left(\nu^{\prime}\right) .
\end{aligned}
$$

The inequalities (4.8) and (4.10) will ensure that the requirements of Theorem 4.5 are met for $\mu^{\prime \prime}$ and $\nu^{\prime \prime}$ on $\mathbb{Z}^{m}$, as long as $\|\hat{\psi}\|_{1} \leq C p^{m}$. Now

$$
\begin{aligned}
\|\hat{\psi}\|_{1} & =\sum_{\vec{\xi} \in \mathbb{Z}_{3 p}^{m}}\left|\sum_{\vec{j} \in \mathbb{Z}_{p}^{m}} \phi(\vec{j}) e(\vec{j} \cdot \vec{\eta}) e\left(\frac{\vec{j} \cdot \vec{\xi}}{3 p}\right)\right| \\
& =\sum_{\vec{\xi} \in \mathbb{Z}_{3 p}^{m}}\left|\sum_{\vec{j} \in[-p, 2 p-1]^{m}} \prod_{i=1}^{m} \varphi\left(j_{i}\right) e\left(j_{i} \eta_{i}\right) e\left(\frac{j_{i} \xi_{i}}{3 p}\right)\right| \\
& =\prod_{i=1}^{m}\left(\sum_{\xi_{i} \in \mathbb{Z}_{3 p}}\left|\sum_{j_{i} \in[-p, 2 p-1]} \varphi\left(j_{i}\right) e\left(j_{i} \eta_{i}\right) e\left(\frac{j_{i} \xi_{i}}{3 p}\right)\right|\right) .
\end{aligned}
$$

It therefore suffices to show that

$$
\sum_{\xi \in \mathbb{Z}_{3 p}}\left|\sum_{j \in[-p, 2 p-1]} \varphi(j) e(j \eta) e\left(\frac{j \xi}{3 p}\right)\right| \leq C p .
$$

For $\xi=0$, we have the trivial bound

$$
\left|\sum_{j \in[-p, 2 p-1]} \varphi(j) e(j \eta)\right| \leq \sum_{j \in[-p, 2 p-1]}|\varphi(j)| \leq 3 p .
$$

For $\xi \neq 0$, we will pursue the required bound using summation by parts. Letting $\Phi(j)=\varphi(j) e(j \eta)$, extended periodically, and noting that $\Delta \Phi(n)=\Phi(n+1)-\Phi(n)$, we have

$$
\begin{aligned}
\sum_{j \in[-p, 2 p-1]} \Phi(j) e\left(\frac{j \xi}{3 p}\right) & =\Phi(2 p)\left(\sum_{j=-p}^{2 p-1} e\left(\frac{j \xi}{3 p}\right)\right)-\sum_{j=-p}^{2 p-1} \Delta \Phi(j) \sum_{n=-p}^{j} e\left(\frac{n \xi}{3 p}\right) \\
& =-\sum_{j=-p}^{2 p-1} \Delta \Phi(j) \frac{e\left(\frac{(j+1) \xi}{3 p}\right)-e\left(\frac{\xi}{3}\right)}{e\left(\frac{\xi}{3 p}\right)-1},
\end{aligned}
$$

since $\Phi(2 p)=0$. 
Further, we have

$$
\begin{aligned}
& -\sum_{j=-p}^{2 p-1} \Delta \Phi(j) \frac{e\left(\frac{(j+1) \xi}{3 p}\right)-e\left(\frac{\xi}{3}\right)}{e\left(\frac{\xi}{3 p}\right)-1} \\
& =-\left(e\left(\frac{\xi}{3 p}\right)-1\right)^{-1}\left(\sum_{j=-p}^{2 p-1} \Delta \Phi(j) e\left(\frac{(j+1) \xi}{3 p}\right)-\sum_{j=-p}^{2 p-1} \Delta \Phi(j) e\left(\frac{\xi}{3}\right)\right) \\
& =-\left(e\left(\frac{\xi}{3 p}\right)-1\right)^{-1}\left(\sum_{j=-p}^{2 p-1} \Delta \Phi(j) e\left(\frac{(j+1) \xi}{3 p}\right)\right. \\
& =-\left(e\left(\frac{\xi}{3 p}\right)-1\right)^{-1} e\left(\frac{\xi}{3 p}\right) \sum_{j=-p}^{2 p-1} \Delta \Phi(j) e\left(\frac{(j) \xi}{3 p}\right) \cdot
\end{aligned}
$$

We now apply summation by parts a second time:

$$
\begin{aligned}
\sum_{j=-p}^{2 p-1} \Delta & \Delta(j) e\left(\frac{(j) \xi}{3 p}\right) \\
& =\Delta \Phi(2 p)\left(\sum_{j=-p}^{2 p-1} e\left(\frac{(j) \xi}{3 p}\right)\right)-\sum_{j=-p}^{2 p-1} \Delta^{2} \Phi(j) \sum_{n=-p}^{j} e\left(\frac{(n) \xi}{3 p}\right) \\
& =-\sum_{j=-p}^{2 p-1} \Delta^{2} \Phi(j) \frac{e\left(\frac{(j) \xi}{3 p}\right)-e\left(\frac{\xi}{3}\right)}{e\left(\frac{\xi}{3 p}\right)-1} \\
& =-\left(e\left(\frac{\xi}{3 p}\right)-1\right) \sum_{j=-p}^{-1} \Delta^{2} \Phi(j) e\left(\frac{(j+1) \xi}{3 p}\right) .
\end{aligned}
$$

We therefore have that

$$
\begin{aligned}
\left|\sum_{j \in[-p, 2 p-1]} \varphi(j) e(j \eta) e\left(\frac{j \xi}{3 p}\right)\right| & =\left|\left(e\left(\frac{\xi}{3 p}\right)-1\right)^{-2} \sum_{j=-p}^{2 p-1} \Delta^{2} \Phi(j) e\left(\frac{(j+2) \xi}{3 p}\right)\right| \\
& \leq\left|\left(e\left(\frac{\xi}{3 p}\right)-1\right)^{-2}\right| \sum_{j=-p}^{2 p-1}\left|\Delta^{2} \Phi(j)\right| .
\end{aligned}
$$

As a consequence,

$$
\sum_{\substack{\xi \in \mathbb{Z}_{3 p} \\ \xi \neq 0}}\left|\sum_{j \in[-p, 2 p-1]} \varphi(j) e(j \eta) e\left(\frac{j \xi}{3 p}\right)\right| \leq\left\|\Delta^{2} \Phi(j)\right\|_{\ell^{1}} \sum_{\substack{\xi \in \mathbb{Z}_{3 p} \\ \xi \neq 0}}\left|\left(e\left(\frac{\xi}{3 p}\right)-1\right)^{-2}\right| .
$$

We will estimate each factor separately, making use of the inequality $|e(\theta)-1|<$ $|2 \pi \theta|$, which is a consequence of the fact that a chord is necessarily shorter than 
the arc it subtends. For the second factor, this gives us

$$
\sum_{\substack{\xi \in \mathbb{Z}_{3 p} \\ \xi \neq 0}}\left|\left(e\left(\frac{\xi}{3 p}\right)-1\right)^{-2}\right|<9 p^{2} \sum_{\xi=1}^{\infty} \frac{1}{(2 \pi \xi)^{2}}=C p^{2} .
$$

For the first, we have

$$
\begin{aligned}
\sum_{j=-p}^{2 p-1}\left|\Delta^{2} \Phi(j)\right| & =\sum_{j=-p}^{2 p-1}|\Phi(j+2)-2 \Phi(j+1)+\Phi(j)| \\
& =\sum_{j=-p}^{2 p-1}|\varphi(j+2) e((j+2) \eta)-2 \varphi(j+1) e((j+1) \eta)+\varphi(j) e(j \eta)| \\
& =\sum_{j=-p}^{2 p-1}|\varphi(j+2) e(2 \eta)-2 \varphi(j+1) e(\eta)+\varphi(j)| .
\end{aligned}
$$

For $j \in\left[-p, \frac{-p-1}{2}-3\right] \cup\left[\frac{3}{2}(p-1), 2 p-1\right]$ this last sum is 0 . For $j \in[0, p-3]$, we have $\varphi(j+2)=\varphi(j+1)=\varphi(j)=1$; hence

$$
\begin{aligned}
|\varphi(j+2) e(2 \eta)-2 \varphi(j+1) e(\eta)+\varphi(j)| & =|e(2 \eta)-2 e(\eta)+1| \\
& =|e(\eta)-1|^{2}<(2 \pi \eta)^{2} .
\end{aligned}
$$

Since $\eta<1 / p$, this is less than $40 / p^{2}$. The sum over this subinterval is therefore less than $\frac{40(p-3)}{p^{2}}<40 / p$.

For $j \in\left[\frac{-p-1}{2}-1,-1\right]$, we have

$$
\begin{aligned}
\mid \varphi(j & +2) e(2 \eta)-2 \varphi(j+1) e(\eta)+\varphi(j) \mid \\
& =\left|\left(\varphi(j)+\frac{4}{p-1}\right) e(2 \eta)-2\left(\varphi(j)+\frac{2}{p-1}\right) e(\eta)+\varphi(j)\right| \\
& <|\varphi(j)| \frac{40}{p^{2}}+\left|\frac{4}{p-1} e(2 \eta)-\frac{4}{p-1} e(\eta)\right| \\
& =|\varphi(j)| \frac{40}{p^{2}}+\frac{4}{p-1}|e(\eta)-1| \\
& <\frac{40+8 \pi}{p^{2}} .
\end{aligned}
$$

A similar calculation gives the same bound over the interval $\left[p-1, \frac{3}{2}(p-1)-2\right]$. Thus we also have a bound of $C / p$ on these sums.

This leaves us with only the cases $j=\frac{-p-1}{2}-2$ and $j=\frac{3}{2}(p-1)-1$. For $j=\frac{-p-1}{2}-2$, though, we have that $\varphi(j)=\varphi(j+1)=0$; this leaves us with

$$
\left|\varphi\left(\frac{-p-1}{2}\right) e(2 \eta)\right|=\frac{2}{p-1} .
$$

The same bound holds for the case $j=\frac{3}{2}(p-1)-1$.

We therefore have that

$$
\sum_{j=-p}^{2 p-1}\left|\Delta^{2} \Phi(j)\right|<\frac{C}{p} .
$$

This completes our estimate (4.11). 
Remark. In the case $q=1$ (recall that $m=d q$ ), we are actually finished, since our desired measure $\mu$ is majorized by $\mu^{\prime \prime}$. We wish to generalize, however, to show that we can achieve any desired polynomial rate of sparsity. Therefore, we shall introduce a second operator to "project down" from $\mathbb{Z}^{m}$ to $\mathbb{Z}^{d}$.

Define the Freiman isomorphism $F: \mathbb{Z}^{m} \rightarrow \mathbb{Z}^{d}$ by

$$
F(\vec{j})=\left(\sum_{i=1}^{q} p^{i-1} j_{i}, \sum_{i=q+1}^{2 q} p^{i-(q+1)} j_{i}, \ldots, \sum_{i=q(d-1)+1}^{d q} p^{i-(q(d-1)+1)} j_{i}\right) .
$$

We note that $F$ maps $[0, p-1]^{m}$ bijectively to $\left[0, p^{q}-1\right]^{d}$, and that for any $k \geq 0$ and any $j_{i} \in[-p, 2 p-1]$ we have $\sum_{i=k q+1}^{(k+1) q} p^{i-1} j_{i} \in\left[-q p^{q}, q p^{q}\right]$; hence, for $\vec{j} \in[-p, 2 p-1]^{m}$, we have

$$
F(\vec{j}) \in\left[-q p^{q}, q p^{q}\right]^{d} .
$$

Define the operator $\Gamma_{2}$ by

$$
\Gamma_{2} f=\sum_{\{\vec{j}: F(\vec{j})=\vec{n}\}} f(\vec{j}) .
$$

Now consider the operator $\Gamma=\Gamma_{2} \Gamma_{1}: \ell^{1}\left(\mathbb{Z}_{p}^{m}\right) \rightarrow \ell^{1}\left(\mathbb{Z}^{d}\right)$. As before, we define our intermediate measures $\mu^{\prime \prime \prime}$ and $\nu^{\prime \prime \prime}$ :

$$
\begin{aligned}
\mu^{\prime \prime \prime} & =\Gamma_{2}\left(\mu^{\prime \prime}\right), \text { and } \\
\nu^{\prime \prime \prime} & =\Gamma_{2}\left(\nu^{\prime \prime}\right),
\end{aligned}
$$

and we want to ensure that $\|\widehat{\Gamma f}\|_{L^{\infty}\left(\mathbb{T}^{d}\right)} \leq C\|\hat{f}\|_{\ell^{\infty}\left(\mathbb{Z}_{p}^{m}\right)}$ so that we may apply Theorem 4.5 .

Now,

$$
\begin{aligned}
\widehat{\Gamma f}(\vec{\theta}) & =\sum_{\vec{n} \in \mathbb{Z}^{d}} \Gamma_{2} \Gamma_{1} f(\vec{n}) e(\vec{\theta} \cdot \vec{n}) \\
& =\sum_{\vec{n} \in \mathbb{Z}^{d}} e(\vec{\theta} \cdot \vec{n}) \sum_{\{\vec{j}: F(\vec{j})=\vec{n}\}} \Gamma_{1} f(\vec{j}) \\
& =\sum_{\vec{j} \in[-p, 2 p-1]^{m}} \Gamma_{1} f(\vec{j}) e\left(\theta_{1} \sum_{i=1}^{q} p^{i-1} j_{i}+\theta_{2} \sum_{i=q+1}^{2 q} p^{i-(q+1)} j_{i}\right. \\
& \left.+\ldots+\theta_{d} \sum_{i=q(d-1)+1}^{d q} p^{i-(q(d-1)+1)} j_{i}\right)
\end{aligned}
$$

So, $\left\|\widehat{\Gamma_{2} \Gamma_{1} f}\right\|_{\ell^{\infty}} \leq\left\|\widehat{\Gamma_{1} f}\right\|_{\ell^{\infty}}$, and by our previous bound we have that $\mu^{\prime \prime \prime}=$ $\Gamma_{2} \Gamma_{1} \mu^{\prime}$ and $\nu^{\prime \prime \prime}=\Gamma_{2} \Gamma_{1} \nu^{\prime}$ obey the difference requirement from Theorem 4.5. Further, we have that

$$
\mu^{\prime \prime \prime}(\vec{n}) \geq \frac{1}{p^{m}} \sum_{\vec{j} \in \bar{S}} \delta_{\vec{j}}(\vec{n})
$$


where

$$
\bar{S}=\left\{\left(\sum_{i=1}^{q} p^{i-1}\left[j^{i}\right]_{p}, \sum_{i=1}^{q} p^{i-1}\left[j^{q+i}\right]_{p}, \ldots, \sum_{i=1}^{q} p^{i-1}\left[j^{(d-1) q+i}\right]_{p}\right): 0 \leq j<p\right\},
$$

so that a weak $(1,1)$ inequality for $\mu^{\prime \prime \prime}$ implies a weak $(1,1)$ inequality for $\mu$. We now wish to show that $\mu^{\prime \prime \prime}$ and $\nu^{\prime \prime \prime}$ satisfy the other two requirements of Theorem 4.5 .

We first observe that

$$
\#\left(\operatorname{supp}\left(\Gamma_{1} \mu^{\prime}\right)\right)=\#\left(\operatorname{supp}\left(\mathbf{1}_{[-p, 2 p-1]^{m}} \phi \circ \tau_{3 p} \mu^{\prime} \circ \tau_{p}\right)\right) \leq C p
$$

Recalling our requirements on our original sequence of primes, and noting that \# $\left(\operatorname{supp}\left(\Gamma_{2} f\right)\right) \leq \#(\operatorname{supp}(f))$, our condition on the support of $\mu^{\prime \prime \prime}$ is satisfied.

For the final requirement, we first note that \# $\left(\operatorname{supp} \Gamma_{1} \nu^{\prime}\right) \leq(3 p)^{m}$; the support of $\nu^{\prime \prime \prime}$, then, likewise has measure less than $C p^{m}$. The supremum of $\nu^{\prime}$ is $1 / p^{m}$. Thus we have

$$
\begin{aligned}
\left\|\nu^{\prime \prime \prime}\right\|_{\ell^{1}} & \leq C p^{m}\left\|\Gamma_{2} \Gamma_{1} \nu^{\prime}\right\|_{\ell^{\infty}} \\
& \leq C p^{m} \sup _{\vec{n}} \sum_{\{\vec{j}: F(\vec{j})=\vec{n}\}} \mathbf{1}_{[-p, 2 p-1]^{m}} \frac{\phi}{p^{m}} \mid \\
& \leq C 3^{m} \sum_{\substack{\{\vec{j}: F(\vec{j})=\vec{n}\} \\
\vec{j} \in[0, p-1]^{m}}} 1 \\
& \leq C 3^{m} .
\end{aligned}
$$

We then have a weak-(1,1) bound for $\sup _{k}|f| *\left|\nu_{k}^{\prime \prime \prime}\right|$.

Restoring the subscripts, define

$$
\begin{aligned}
& \mu_{k}(\vec{n})=\mu_{k}^{\prime \prime \prime}\left(\vec{k}-\vec{a}_{k}\right), \text { and } \\
& \nu_{k}(\vec{n})=\nu_{k}^{\prime \prime \prime}\left(\vec{k}-\vec{a}_{k}\right) .
\end{aligned}
$$

By Theorem 4.5 $\sup _{k}\left|f * \mu_{k}\right|$ obeys a weak-(1,1) inequality. Together with inequality (4.14), this completes the proof.

4.1.2. Proof of Theorem 4.3. As in Section 4.1.1, we will use the exceptionally good Fourier bounds of certain measures on $\mathbb{Z}_{p}^{m}$, and transfer these to measures on $\mathbb{Z}^{d}$ using operators $\Gamma_{p}$. However, in this case we cannot allow ourselves to use a smooth cutoff function, because we need to wind up with the actual averages, not simply weighted averages which majorize them. This introduces a logarithmic factor which would have been fatal to the weak $L^{1}$ maximal inequality, but which is harmless here.

To begin, given the sequence $n_{i}=a_{k}+x_{k, j}$ (where of course $0 \leq j<p_{k}$ ) in the dictionary ordering (and thus given indices $k(i)$ and $j(i)$ for each $i \in \mathbb{N}$ ), we define 
the measures on $\mathbb{Z}^{d}$ :

$$
\begin{aligned}
& \mu_{N}=\frac{1}{N} \sum_{i=1}^{N} \delta_{n_{i}}, \\
& \nu_{N}=\frac{1}{N}\left(\frac{j(N)}{p_{k(N)}^{m}} \chi\left(\left[0, p_{k(N)}^{q}-1\right]^{d}\right)+\sum_{k=1}^{k(N)-1} p_{k}^{1-m} \chi\left(\left[0, p_{k}^{q}-1\right]^{d}\right)\right) .
\end{aligned}
$$

$\mu_{N}$ simply corresponds to an average over our sparse sequence, while $\nu_{N}$ is a weighted average over the $d$-dimensional blocks which our sequence "lives on". We will see that $\mu_{N}$ and $\nu_{N}$ are made from the images under the operators $\Gamma_{p}$ of the measures in (4.7) and (4.9) on $\mathbb{Z}_{p_{k}}^{m}$, and thus we may count on their Fourier transforms to be very close to one another.

Recalling the Freiman isomorphisms $F_{p}: \mathbb{Z}^{m} \rightarrow \mathbb{Z}^{d}$ from (4.13), and identifying $Z_{p}^{m}$ with $[0, p-1]^{m} \subset \mathbb{Z}^{m}$, define $\Gamma_{p}: \ell^{1}\left(\mathbb{Z}_{p}^{m}\right) \rightarrow \ell^{1}\left(\mathbb{Z}^{d}\right)$ by

$$
\Gamma_{p}(f)(\vec{n})=f\left(F_{p}^{-1}(\vec{n})\right) \chi_{\left[0, p^{q}-1\right]^{d}}(\vec{n}) .
$$

Here $\left(F_{p}^{-1}(\vec{v})\right.$ denotes, for $\vec{v} \in\left[0, p^{q}-1\right]^{d}$, the unique $\vec{w} \in \mathbb{Z}^{m}$ such that $F_{p}(\vec{w})=\vec{v}$.) This is the same as the final operator from the last section, except that we do not use a smooth cutoff function.

By the same argument, we may conclude that

$$
\left\|\widehat{\Gamma_{p} f}\right\|_{L^{\infty}\left(\mathbb{T}^{d}\right)} \leq C p^{-m}\|\hat{\psi}\|_{\ell^{1}\left(\mathbb{Z}_{p}^{m}\right)}\|\hat{f}\|_{\ell^{\infty}\left(\mathbb{Z}_{p}^{m}\right)}
$$

where now $\psi(\vec{n})=e(\vec{n} \cdot \vec{\eta})$ for some $\vec{\eta} \in \mathbb{T}^{m}$ with $\left|\eta_{i}\right|<1 / p$ for $i=1, \ldots, m$. But trivially, $\|\hat{\psi}\|_{\ell^{1}\left(\mathbb{Z}_{p}^{m}\right)} \leq(C p \log p)^{m}$, so $\left\|\widehat{\Gamma_{p} f}\right\|_{L^{\infty}\left(\mathbb{T}^{d}\right)} \leq C(\log p)^{m}\|\hat{f}\|_{\ell^{\infty}\left(\mathbb{Z}_{p}^{m}\right)}$.

For all of the pieces of $\mu_{N}$ and $\nu_{N}$ with $k<k(N)$, we may of course apply Weil's theorem on complete character sums (Theorem 4.1). However, the last component corresponds to an incomplete character sum, for which we instead apply Weyl's Inequality 25] to improve on the trivial bound; we find overall that

$$
\left\|\hat{\mu}_{N}-\hat{\nu}_{N}\right\|_{L^{\infty}\left(\mathbb{T}^{d}\right)} \leq C N^{-\epsilon}
$$

for some $\epsilon>0$. Thus along any lacunary $I \subset \mathbb{N}$, we see that

$$
\sum_{t \in I}\left\|\left(\mu_{t}-\nu_{t}\right) * f\right\|_{\ell^{2}\left(\mathbb{Z}^{d}\right)}^{2} \leq C\|f\|_{\ell^{2}\left(\mathbb{Z}^{d}\right)}^{2} .
$$

Therefore an oscillation inequality for convolution with $\nu_{t}$ would imply (4.4).

As in [18, we introduce simple Fourier multiplier operators $V_{t}$ on $\ell^{2}\left(\mathbb{Z}^{d}\right)$, defined by

$$
\hat{V}_{t} f(\vec{\alpha})= \begin{cases}\hat{f}(\vec{\alpha}), & |\alpha| \leq p_{k(t)}^{-1}, \\ 0 & \text { otherwise. }\end{cases}
$$

Now we can pass from an oscillational inequality for $\nu_{t}$ to that for $V_{t}$, because

Lemma 4.6.

$$
\sum_{t \in I}\left\|\nu_{t} * f-V_{t} f\right\|_{2}^{2} \leq C\|f\|_{2}^{2}
$$

Proof. This follows from the assertion

$$
\sup _{\alpha \in \mathbb{T}^{d}}\left(\sum_{t \in I}\left|\hat{\nu}_{t}(\alpha)-\hat{V}_{t}(\alpha)\right|\right) \leq C .
$$


Fix $\alpha \in \mathbb{T}^{d}$, and take $K$ such that $|\alpha| \approx p_{K}^{-1}$. Then for $t$ with $k(t)<K$, a simple calculation shows that

$$
\left|\hat{\nu}_{t}(\alpha)-\hat{V}_{t}(\alpha)\right|=\left|\hat{\nu}_{t}(\alpha)-1\right| \leq|\alpha|\left\|\nabla \hat{\nu}_{t}\right\|_{\infty} \lesssim \frac{p_{k(t)}}{p_{K}} .
$$

Note that the calculation of $\left\|\nabla \hat{\nu}_{t}\right\|_{\infty}$ is the one and only place we use the growth assumption on $\vec{a}_{k}$.

For $t$ with $k(t)>K$,

$$
\left|\hat{\nu}_{t}(\alpha)-\hat{V}_{t}(\alpha)\right|=\left|\hat{\nu}_{t}(\alpha)\right| \lesssim \frac{1}{t} \prod_{i=1}^{d} \frac{1}{\left|\alpha_{i}\right|+1} \lesssim \frac{p_{K}}{p_{k(t)}} .
$$

This calculation uses the fact that $\hat{\nu}_{t}(\alpha)$ can be expressed as a weighted sum of averages over blocks which grow exponentially in size. Thus

$$
\left(\sum_{t \in I}\left|\hat{A}_{t}(\alpha)-\hat{V}_{t}(\alpha)\right|\right) \lesssim \sum_{t \in I: k(t)<K} \frac{p_{k(t)}}{p_{K}}+O(1)+\sum_{t \in I: k(t)>K} \frac{p_{K}}{p_{k(t)}} \leq C,
$$

since $p_{k}$ are an exponentially increasing sequence and $I$ is lacunary (thus the number of $t$ associated to any $k$ is uniformly bounded).

We now need only to prove that

$$
\sum_{n}\left\|\sup _{t_{n-1} \leq t \leq t_{n}, t \in I}\left|V_{t} f-V_{t_{n}} f\right|\right\|_{2}^{2} \leq C\|f\|_{2}^{2}
$$

Now we note that for $t_{n-1} \leq t \leq t_{n}, V_{t} f-V_{t_{n}} f=V_{t}\left(V_{t_{n-1}} f-V_{t_{n}} f\right)$. Also, Lemma 4.6 lets us derive an $\ell^{2}$ maximal theorem for $V_{t}$ from the one for $A_{t}$; this follows from our $\ell^{1}$ maximal inequality. Therefore

$$
\begin{aligned}
\left\|\sup _{t_{n-1} \leq t \leq t_{n}, t \in I}\left|V_{t} f-V_{t_{n}} f\right|\right\|_{2}^{2} & =\left\|\sup _{t_{n} \leq t \leq t_{n+1}, t \in I}\left|V_{t}\left(V_{t_{n-1}} f-V_{t_{n}} f\right)\right|\right\|_{2}^{2} \\
& \leq C\left\|V_{t_{n-1}} f-V_{t_{n}} f\right\|_{2}^{2} .
\end{aligned}
$$

Now we see that

$$
\sum_{n}\left\|V_{t_{n-1}} f-V_{t_{n}} f\right\|_{2}^{2} \leq\left(\sup _{\alpha \in \mathbb{T}^{d}} \sum_{n}\left|\hat{V}_{t_{n-1}}(\alpha)-\hat{V}_{t_{n}}(\alpha)\right|\right) \sum_{n}\|f\|_{2}^{2}=\|f\|_{2}^{2}
$$

since the functions $\hat{V}_{t_{n-1}}-\hat{V}_{t_{n}}$ have disjoint supports. This concludes the proof of Theorem 4.3

4.2. The product construction. Suppose that $m=q d$ and $S_{1}, S_{2}, \ldots, S_{r}$ are subsets of $\mathbb{Z}^{d}$, respectively, as constructed above. That is, $S_{i}=\bigcup_{k=1}^{\infty} S_{i, k}$, where

$$
\begin{aligned}
& S_{i, k}=\left\{\vec{a}_{i, k}+\left(\sum_{t=1}^{q} p_{i, k}^{t-1}\left[j_{i}^{t}\right]_{p_{i, k}}, \sum_{t=1}^{q} p_{i, k}^{t-1}\left[j_{i}^{q+t}\right]_{p_{i, k}}, \ldots, \sum_{t=1}^{q} p_{i, k}^{t-1}\left[j_{i}^{(d-1) q+t}\right]_{p_{i, k}}\right)\right. \\
& \left.: 0 \leq j_{i}<p_{i, k}\right\},
\end{aligned}
$$

and $1 \leq i \leq r$. The sequences $\left\{\vec{a}_{i, k}\right\}_{k=1}^{\infty}$ and $\left\{p_{i, k}\right\}_{k=1}^{\infty}$ are not necessarily distinct with respect to $i$.

Let $S=\prod_{i=1}^{r} S_{i}$, and let $B_{t}$ denote the ball of radius $t$ in $\mathbb{Z}^{r d}$. 
Proposition 4.7. Let $S$ be defined as above. Then the maximal function

$$
M f(\vec{n})=\sup _{t} \frac{1}{\#\left(S \cap B_{t}\right)} \sum_{\vec{m} \in S \cap B_{t}}|f(\vec{n}+\vec{m})|
$$

satisfies a weak-(1,1) inequality; that is, there is a constant $C$ so that for any $\lambda>0$ we have

$$
\#\{\vec{n}: M f(\vec{n})>\lambda\}<\frac{C}{\lambda}\|f\|_{\ell^{1}\left(\mathbb{Z}^{r d}\right)}
$$

As in the previous section, we will require two inequalities relating convolutions, products, and norms of Fourier transforms on these finite groups. With the Fourier transform on $\prod_{i=1}^{r} \mathbb{Z}_{p_{i}}^{m}$ defined by

$$
\hat{f}(\vec{\xi})=\sum_{\vec{n} \in \prod_{i=1}^{r} \mathbb{Z}_{p_{i}}^{m}} f(\vec{n})\left(\frac{\vec{n}_{1} \cdot \vec{\xi}_{1}}{p_{1}}+\ldots+\frac{\vec{n}_{r} \cdot \vec{\xi}_{r}}{p_{r}}\right)
$$

where $\vec{n}_{i}$ and $\vec{\xi}_{i}$ are elements of $\mathbb{Z}_{p_{i}}^{m}$, we have the natural analogues of the inequalities (4.5) and (4.6):

$$
\begin{aligned}
\|\widehat{f g}\|_{\ell^{\infty}} & \leq \frac{1}{\prod_{i=1}^{r} p_{i}^{m}}\|\hat{f}\|_{\ell^{1}}\|\hat{g}\|_{\ell^{\infty}}, \text { and } \\
\|f * g\|_{\ell^{2}} & \leq\|f\|_{\ell^{2}}\|\hat{g}\|_{\ell^{\infty}} .
\end{aligned}
$$

Proof. The proof proceeds in very much the same way as that of Theorem 4.4

Once again suppressing our $k$ subscripts, let $p_{1}, p_{2}, \ldots, p_{r}$ be odd primes, each larger than $m \geq 1$. Define

$$
\mu_{r}^{\prime}=\frac{1}{\prod_{i=1}^{r} p_{i}} \sum_{\substack{\left(j_{1}, j_{2}, \ldots, j_{r}\right) \\ \in \prod_{i=1}^{r}\left[0, p_{i}-1\right]}} \delta_{\left(j_{1}, j_{1}^{2}, \ldots, j_{1}^{m} ; j_{2}, j_{2}^{2}, \ldots, j_{2}^{m} ; \ldots ; j_{r}, j_{r}^{2}, \ldots, j_{r}^{m}\right)},
$$

noting that

$$
\#\left(\operatorname{supp}\left(\mu_{r}^{\prime}\right)\right)=\prod_{i=1}^{r} p_{i}
$$

We also define

$$
\nu_{r}^{\prime}=\left(\prod_{i=1}^{r} p_{i}\right)^{-m} \sum_{\vec{j} \in \prod_{i=1}^{r} \mathbb{Z}_{p_{i}}^{m}} \delta_{\vec{j}}
$$

As before, we will first seek an appropriate bound on $\left|\widehat{\mu_{r}^{\prime}-\nu_{r}^{\prime}}\right|$. 
As our products remain finite abelian groups, we have that the Fourier transform of $\mu_{r}^{\prime}$ may be written as

$$
\begin{aligned}
\hat{\mu_{r}^{\prime}(\vec{\theta})=} & \sum_{\vec{n} \in \prod_{i=1}^{r} \mathbb{Z}_{p_{i}}^{m}} \mu_{r}^{\prime}(\vec{n}) e\left(\frac{\vec{n}_{1} \cdot \vec{\theta}_{1}}{p_{1}}+\frac{\vec{n}_{2} \cdot \vec{\theta}_{2}}{p_{2}}+\ldots+\frac{\vec{n}_{r} \cdot \vec{\theta}_{r}}{p_{r}}\right) \\
= & \frac{1}{\prod_{i=1}^{r} p_{i}} \sum_{\substack{\left(j_{1}, j_{2}, \ldots, j_{r}\right) \\
\in \prod_{i=1}^{r}\left[0, p_{i}-1\right]}} \sum_{\vec{n} \in \prod_{i=1}^{r} \mathbb{Z}_{p_{i}}^{m}} \\
& \times \delta_{\left(j_{1}, \ldots, j_{1}^{m} ; \ldots ; j_{r}, \ldots, j_{r}^{m}\right)}(\vec{n}) e\left(\frac{\vec{n}_{1} \cdot \vec{\theta}_{1}}{p_{1}}\right) e\left(\frac{\vec{n}_{2} \cdot \vec{\theta}_{2}}{p_{2}}\right) \ldots e\left(\frac{\vec{n}_{r} \cdot \vec{\theta}_{r}}{p_{r}}\right) \\
= & \frac{1}{\prod_{i=1}^{r} p_{i}} \sum_{j_{1}=0}^{p_{1}-1} \ldots \sum_{j_{r}=0}^{p_{r}-1} e\left(\frac{j_{1} \theta_{1}+j_{1}^{2} \theta_{2}+\ldots+j_{1}^{m} \theta_{m}}{p_{1}}\right) \\
& \ldots e\left(\frac{j_{r} \theta_{(r-1) m+1}+j_{r}^{2} \theta_{(r-1) m+2}+\ldots+j_{r}^{m} \theta_{r m}}{p_{r}}\right) \\
= & \prod_{i=1}^{r}\left(\frac{1}{p_{i}} \sum_{j_{i}=0}^{p_{i}-1} e\left(\frac{j_{i} \theta_{(i-1) m+1}+j_{i}^{2} \theta_{(i-1) m+2}+\ldots+j_{i}^{m} \theta_{i m}}{p_{i}}\right)\right),
\end{aligned}
$$

where $\overrightarrow{n_{i}}$ denotes those entries of $\vec{n}$ drawn from $\mathbb{Z}_{p_{i}}$ and

$$
\vec{\theta}_{i}=\left(\theta_{(i-1) m+1}, \theta_{(i-1) m+2}, \ldots, \theta_{i m}\right) .
$$

By Theorem 4.1 we must then have

$$
\left|\hat{\mu_{r}^{\prime}}\right| \leq \frac{(m-1)^{r}}{\prod_{i=1}^{r} p_{i}^{1 / 2}}
$$

We have that

$$
\hat{\nu_{r}^{\prime}}(\vec{\theta})=\left\{\begin{array}{rr}
1 & \text { if } \vec{\theta}=\overrightarrow{0} \\
0 & \text { otherwise }
\end{array}\right.
$$

and that $\hat{\mu_{r}^{\prime}}(\overrightarrow{0})=1$. Therefore,

$$
\left|\widehat{\mu_{r}^{\prime}-\nu_{r}^{\prime}}\right| \leq \frac{(m-1)^{r}}{\prod_{i=1}^{r} p_{i}^{1 / 2}}
$$

We now embark on the construction of $\Gamma_{1}$ and $\Gamma_{2}$.

Identifying $\mathbb{Z}_{3 p_{i}}^{m}$ with $\left[-p_{i}, 2 p_{i}-1\right]^{m}$ and $\mathbb{Z}_{3 p_{i}}$ with $\left[-p_{i}, 2 p_{i}-1\right]$, we define the functions $\varphi_{i}: \mathbb{Z}_{3 p_{i}} \rightarrow \mathbb{R}$ by

$$
\varphi_{i}(n)= \begin{cases}1 & \text { if } n \in\left[0, p_{i}-1\right], \\ 0 & \text { if } n \in\left[-p_{i}, \frac{-p_{i}-1}{2}\right] \cup\left[\frac{3}{2}\left(p_{i}-1\right), 2 p_{i}-1\right], \text { and } \\ \text { affine } & \text { otherwise. }\end{cases}
$$

With $\vec{n}_{i}=\left(n_{i, 1}, n_{i, 2}, \ldots, n_{i, m}\right)$, we then define $\phi: \prod_{i=1}^{r} \mathbb{Z}_{3 p_{i}}^{m} \rightarrow \mathbb{R}$ by

$$
\phi(\vec{n})=\prod_{i=1}^{r} \prod_{k=1}^{m} \phi_{i}\left(n_{i, k}\right) .
$$


Define $\tau_{p}: \mathbb{Z}^{r m} \rightarrow \prod_{i=1}^{r} \mathbb{Z}_{p_{i}}^{m}$ and $\tau_{3 p}: \mathbb{Z}^{r m} \rightarrow \prod_{i=1}^{r} \mathbb{Z}_{3 p_{i}}^{m}$ by

$$
\begin{gathered}
\tau_{p}(\vec{k})=\left(\left[k_{1}\right]_{p_{1}},\left[k_{2}\right]_{p_{1}}, \ldots,\left[k_{m}\right]_{p_{1}},\left[k_{m+1}\right]_{p_{2}},\left[k_{m+2}\right]_{p_{2}}, \ldots,\left[k_{2 m}\right]_{p_{2}},\right. \\
\left.\ldots,\left[k_{(r-1) m+1}\right]_{p_{r}}, \ldots,\left[k_{r m}\right]_{p_{r}}\right), \text { and } \\
\tau_{3 p}(\vec{k})=\left(\left[k_{1}\right]_{3 p_{1}},\left[k_{2}\right]_{3 p_{1}}, \ldots,\left[k_{m}\right]_{3 p_{1}},\left[k_{m+1}\right]_{3 p_{2}},\left[k_{m+2}\right]_{3 p_{2}}, \ldots,\left[k_{2 m}\right]_{3 p_{2}},\right. \\
\left.\ldots,\left[k_{(r-1) m+1}\right]_{3 p_{r}}, \ldots,\left[k_{r m}\right]_{3 p_{r}}\right)
\end{gathered}
$$

We now define $\Gamma_{1}$ :

$$
\Gamma_{1}(f)=\mathbf{1}_{\prod_{i=1}^{r}\left[-p_{i}, 2 p_{i}-1\right]^{m}} \phi \circ \tau_{3 p} f \circ \tau_{p} .
$$

Suppose $\vec{\theta} \in \mathbb{T}^{r m}$ and let $\vec{\xi}_{i} \in \mathbb{Z}^{m}$ so that

$$
\vec{\theta}=\left(\vec{\xi}_{1} / 3 p_{1}+\vec{\eta}_{1}, \vec{\xi}_{2} / 3 p_{2}+\vec{\eta}_{2}, \ldots, \vec{\xi}_{r} / 3 p_{r}+\vec{\eta}_{r}\right),
$$

with $\left|\eta_{i, k}\right| \leq C / p_{i}$ for all $k$ and $i$.

If $f: \prod_{i=1}^{r} \mathbb{Z}_{p_{i}}^{m} \rightarrow \mathbb{R}$, then the Fourier transform of $\Gamma_{1} f$ would be

$$
\begin{aligned}
& \widehat{\Gamma_{1} f}(\vec{\theta})=\sum_{\vec{j} \in \mathbb{Z}^{r m}} \Gamma_{1} f(\vec{j}) e(\vec{j} \cdot \vec{\theta}) \\
& =\sum_{\vec{j} \in \prod_{i=1}^{r}\left[-p_{i}, 2 p_{i}-1\right]^{m}} \phi(\vec{j}) f \circ \tau_{p}(\vec{j}) e\left(\vec{j}_{1} \cdot \vec{\eta}_{1}+\ldots+\vec{j}_{r} \cdot \vec{\eta}_{r}\right) e\left(\vec{j}_{1} \cdot \frac{\vec{\xi}_{1}}{3 p_{1}}+\ldots+\vec{j}_{r} \cdot \frac{\vec{\xi}_{r}}{3 p_{r}}\right) .
\end{aligned}
$$

Letting

$$
\psi(\vec{x})=\phi(\vec{x}) e\left(\vec{x}_{1} \cdot \vec{\eta}_{1}+\ldots+\vec{x}_{r} \cdot \vec{\eta}_{r}\right),
$$

we find that the expression (4.21) is the Fourier transform of the product of $f$ and $\psi$ on $\prod_{i=1}^{r} \mathbb{Z}_{3 p_{i}}^{m}$.

By (4.18), we have that

$$
\left\|\widehat{\Gamma_{1}(f)}(\vec{\theta})\right\|_{\ell^{\infty}} \leq \frac{1}{\prod_{i=1}^{r} p_{i}^{m}}\|f\|_{\ell^{\infty}}\|\hat{\psi}\|_{\ell^{1}} .
$$

In this case, we have that

$$
\begin{aligned}
& \|\hat{\psi}\|_{\ell^{1}}=\sum_{\vec{\xi} \in \prod_{i=1}^{r} \mathbb{Z}_{3 p_{i}}^{m}} \mid \sum_{\vec{j} \in \prod_{i=1}^{r}\left[-p_{i}, 2 p_{i}-1\right]^{m}} \phi(\vec{j}) e\left(\vec{j}_{1} \cdot \vec{\eta}_{1}+\ldots+\vec{j}_{r} \cdot \vec{\eta}_{r}\right) \\
& \times e\left(\frac{\vec{j}_{1} \cdot \vec{\xi}_{1}}{3 p_{1}}+\ldots+\frac{\vec{j}_{r} \cdot \vec{\xi}_{r}}{3 p_{r}}\right) \\
& =\sum_{\vec{\xi} \in \prod_{i=1}^{r} \mathbb{Z}_{3 p_{i}}^{m}} \sum_{\vec{j} \in \prod_{i=1}^{r}\left[-p_{i}, 2 p_{i}-1\right]^{m}}\left(\prod_{i=1}^{r} \prod_{k=1}^{m} \varphi\left(j_{i, k}\right)\right) \\
& \times e\left(\vec{j}_{1} \cdot \vec{\eta}_{1}+\ldots+\vec{j}_{r} \cdot \vec{\eta}_{r}\right) e\left(\frac{\vec{j}_{1} \cdot \vec{\xi}_{1}}{3 p_{1}}+\ldots+\frac{\vec{j}_{r} \cdot \vec{\xi}_{r}}{3 p_{r}}\right) \mid \\
& =\prod_{i=1}^{r} \prod_{k=1}^{m} \sum_{\xi_{i, k} \in \mathbb{Z}_{3 p_{i}}}\left|\sum_{j_{i, k} \in\left[-p_{i}, 2 p_{i}-1\right]} \varphi\left(j_{i, k}\right) e\left(j_{i, k} \eta_{i, k}\right) e\left(\frac{j_{i, k} \xi_{i, k}}{3 p_{i}}\right)\right| .
\end{aligned}
$$


If

$$
\sum_{\xi_{i, k} \in \mathbb{Z}_{3 p_{i}}}\left|\sum_{j_{i, k} \in\left[-p_{i}, 2 p_{i}-1\right]} \varphi\left(j_{i, k}\right) e\left(j_{i, k} \eta_{i, k}\right) e\left(\frac{j_{i, k} \xi_{i, k}}{3 p_{i}}\right)\right| \leq C p_{i},
$$

then we are done; but this is precisely the inequality (4.11).

Suppose $m=q d$. Define $F: \mathbb{Z}^{r m} \rightarrow \mathbb{Z}^{r d}$ by

$$
\begin{aligned}
F(\vec{j})=\left(\sum_{k=1}^{q} p_{1}^{k-1} j_{1, k}, \ldots, \sum_{k=q(d-1)+1}^{d q} p_{1}^{k-(q(d-1)+1)} j_{1, k}, \sum_{k=1}^{q} p_{2}^{k-1} j_{2, k}, \ldots,\right. & \\
& \left.\sum_{k=q(d-1)+1}^{d q} p_{r}^{k-(q(d-1)+1)} j_{r, k}\right) .
\end{aligned}
$$

Once again $F$ acts as a bijection on our sets of interest; here we have that $F$ is a bijection from $\prod_{i=1}^{r}\left[0, p_{i}-1\right]^{m}$ to $\prod_{i=1}^{r}\left[0, p_{i}^{m}-1\right]$. We again define $\Gamma_{2}$ by

$$
\Gamma_{2} f(\vec{n})=\sum_{\{\vec{j}: F(\vec{j})=\vec{n}\}} f(\vec{j}) .
$$

As in Section 3.1, we have that

$$
\left\|\widehat{\Gamma_{2} \Gamma_{1} f}\right\|_{\ell^{\infty}} \leq\left\|\widehat{\Gamma_{1} f}\right\|_{\ell^{\infty}} .
$$

Setting $\mu_{r}^{\prime \prime \prime}=\Gamma_{2} \Gamma_{1} \mu_{r}^{\prime}$ and $\nu_{r}^{\prime \prime \prime}=\Gamma_{2} \Gamma_{1} \nu_{r}^{\prime}$, we then have that $\mu_{r}^{\prime \prime \prime}$ and $\nu_{r}^{\prime \prime \prime}$ meet the difference requirement of Theorem 4.5 .

We also have that

$$
\#\left(\operatorname{supp}\left(\Gamma_{2} \Gamma_{1} \mu_{r}^{\prime}\right)\right) \leq \#\left(\operatorname{supp}\left(\Gamma_{1} \mu_{r}^{\prime}\right)\right) \leq C \prod_{i=1}^{r} p_{i},
$$

and that

$$
\begin{aligned}
\left\|\nu_{r}^{\prime \prime \prime}\right\|_{\ell^{1}} & \leq \#\left(\operatorname{supp}\left(\nu_{r}^{\prime \prime \prime}\right)\right)\left\|\Gamma_{2} \Gamma_{1} \nu_{r}^{\prime}\right\|_{\ell^{\infty}} \\
& \leq C \prod_{i=1}^{r} p_{i}^{m} \sup _{\vec{n}}\left|\sum_{\{\vec{j}: F(\vec{j})=\vec{n}\}} \frac{\mathbf{1}_{\prod_{i=1}^{r}\left[-p_{i}, 2 p_{i}-1\right]^{m}} \phi \circ \tau_{3 p}}{\prod_{i=1}^{r} p_{i}^{m}}\right| \\
& \leq C .
\end{aligned}
$$

As in the previous section, then, all three requirements will be satisfied; noting that

$$
\mu_{r}^{\prime \prime \prime}(\vec{n}) \geq \frac{1}{\prod_{i=1}^{r} p_{i}} \sum_{\vec{j} \in \tilde{S}} \delta_{\vec{j}}(\vec{n})
$$

where

$$
\begin{array}{r}
\tilde{S}=\left\{\left(\sum_{t=1}^{q} p_{1, k}^{t-1}\left[j_{1}^{t}\right]_{p_{1, k}}, \ldots, \sum_{t=1}^{q} p_{1, k}^{t-1}\left[j_{1}^{(d-1) q+t}\right]_{p_{1, k}}, \ldots, \sum_{t=1}^{q} p_{r, k}^{t-1}\left[j_{r}^{(d-1) q+t}\right]_{p_{r, k}}\right)\right. \\
\left.: 0 \leq j_{i}<p_{i}, 1 \leq i \leq r\right\}
\end{array}
$$

it remains only to reintroduce our subscripts and to shift by $\vec{a}_{i, k}$. 
Defining $\mu_{k}$ and $\nu_{k}$ by

$$
\begin{aligned}
\mu_{k}(\vec{n}) & =\mu_{r, k}^{\prime \prime \prime}(\vec{n}), \\
\nu_{k}(\vec{n}) & =\nu_{r, k}^{\prime \prime \prime}(\vec{n}) ;
\end{aligned}
$$

we have that $\sup _{k}\left|f * \mu_{k}\right|$ obeys a weak-(1,1) inequality. With this and the inequality (4.22), the proof is complete.

We have that a product of sum sets of the type constructed in Section 3.1 remains a good sum set.

\section{Sparse SEqUENCES AND ACtions of VIRTUALly Nilpotent GROUPS}

We begin with a few necessary definitions.

Definition. Let $G$ be an infinite finitely generated group with identity $e$, and $\mathbb{A}=\left\{e, a_{1}, \ldots, a_{n}\right\} \subset G$ be a finite symmetric generating set containing $e$. Let $\mathbb{A}^{N}$ denote the elements of $G$ expressible as words of length $N$ in $\mathbb{A}$, and let $\mathbb{A}^{0}:=\{e\}$. Then $\rho^{\mathbb{A}}(g, h):=\min \left\{N: g h^{-1} \in \mathbb{A}^{N}\right\}$ defines a metric on $G$.

Select a symmetric set of generators $\mathbb{A}$. Then $\mathbb{A}^{N}$ is the ball of radius $N$ in the word metric on $G$. Classical results by Wolf [26, Bass [1, Milnor [16] and Gromov [12] amount to the following: $G$ is virtually nilpotent (contains a nilpotent subgroup of finite index) if and only if there exist $d \in \mathbb{N}$ and $0<c<C<\infty$ such that for all $N \in \mathbb{Z}^{+}$,

$$
c N^{d}<\# \mathbb{A}^{N} \leq C N^{d}
$$

(here $C$, but not $d$, depends on the choice of $\mathbb{A}$ ). Thus we say that $G$ has polynomial growth of degree $d$.

Pansu [17] improved this result further:

Theorem 5.1. Let $\mathbb{A}$ be a symmetric set of generators for the virtually nilpotent group $G$. Then there is an integer $d$ so that the sequence

$$
\frac{\# \mathbb{A}^{N}}{N^{d}}
$$

converges.

Note that in particular this implies, for all $g \in G$,

$$
\lim _{N \rightarrow \infty} \frac{\#\left(\mathbb{A}^{N} \Delta g \mathbb{A}^{N}\right)}{\# \mathbb{A}^{N}}=0 .
$$

Since the important matters in the proofs that follow do not depend on our choice of $\mathbb{A}$, we will henceforward suppress it in the superscripts.

5.1. Block averages for virtually nilpotent groups. In the proof below, we will again seek to apply Tempelman's Theorem to a shifted sequence of sets whose volume increases in a lacunary fashion. Instead of rectangular prisms, however, we will take as our shifted sets elements of the family $\left\{\mathbb{A}^{N}\right\}$.

We immediately note that for any particular radius, the volume of the corresponding ball is finite, and that for any $m>n, \mathbb{A}^{n} \subset \mathbb{A}^{m}$. Further, the family of balls of radius $N,\left\{\mathbb{A}^{N}\right\}_{N>0}$, themselves satisfy the Følner condition (see [6]). 
Choose the sequence $\ell_{k}$ as in Section 2.2, and a sequence of elements $a_{k} \in G$, with

(1) $\rho\left(a_{k+1}, e\right)>\rho\left(a_{k}, e\right)+\ell_{k}$, and

(2) $\ell_{k} \geq C \rho\left(a_{k-1}, e\right)$.

Defining $B_{k}=\mathbb{A}^{\ell_{k}}$, we let $S=\bigcup_{k>0} a_{k} B_{k}$.

Suppose

where $0 \leq r<\ell_{k}$.

$$
S(k, r)=\left(\bigcup_{i<k+1} a_{i} B_{i}\right) \cup a_{k+1} \mathbb{A}^{r}
$$

Proposition 5.2. The sequence $S(k, r)$, with $k \geq 1$ and $r \geq 0$, is a pointwise $L^{1}$-good sequence of sets for any free $G$-action.

Remark. For the proposition as it is written, merely having the upper and lower bound (as in Section 5.1) would suffice; however, Theorem 5.1 gives us more. Specifically, we have that an average over any increasing sequence of sets of which our Følner sequence is a subsequence must also converge. For example, we have that the averages taken element-by-element also converge, as long as we successively fill each set in our constructed sequence. A similar result holds in the $\mathbb{Z}^{d}$ case, as an immediate corollary.

Note that if we consider only $r=0$, we would have an $L^{1}$-good sequence corresponding to the sequence consisting only of whole blocks in the original block sequence construction.

Proof. As in Section 2, we need only verify the difference requirement.

Letting $S_{k}=\bigcup_{i<k+1} a_{i} B_{i}$ and $R=a_{k+1} \mathbb{A}^{r}$, we have

$$
\#\left(S(k, r) S^{-1}(k, r)\right) \leq \#\left(S_{k} S_{k}^{-1}\right)+\#\left(S_{k} R^{-1}\right)+\#\left(R S_{k}^{-1}\right)+\#\left(R R^{-1}\right) .
$$

We note that for any ball $\mathbb{A}^{N}$ in $G$, we have

$$
\mathbb{A}^{N}\left(\mathbb{A}^{N}\right)^{-1} \subseteq \mathbb{A}^{2 N}
$$

Hence, due to the polynomial growth of $G$, the size of the difference of any ball with itself is bounded by the size of the original ball:

$$
\#\left(\mathbb{A}^{N}\left(\mathbb{A}^{N}\right)^{-1}\right) \leq \# \mathbb{A}^{2 N} \leq C(2 N)^{d} \leq 2^{d} \# \mathbb{A}^{N} .
$$

Thus the last term in (5.3) is less than $C \# R$ for some constant $C$.

For the first term, we again consider a decomposition:

$$
\begin{aligned}
\#\left(S_{k} S_{k}^{-1}\right) \leq & \#\left(a_{k} B_{k} B_{k}^{-1} a_{k}^{-1}\right)+\#\left(a_{k} B_{k} S_{k-1}^{-1}\right)+\#\left(S_{k-1} B_{k}^{-1} a_{k}^{-1}\right) \\
& +\#\left(S_{k-1} S_{k-1}^{-1}\right) .
\end{aligned}
$$

Again, we immediately have that the first term is less than $C \# S_{k}$. But $S_{k-1} \subset B_{k}$, by our condition on $\ell_{k}$. So each of the three other terms is also less than $C \# S_{k}$.

This leaves only the second and third terms of (5.3). We note that, by our conditions on $\ell_{k}, S_{k} \subseteq \mathbb{A}^{c \ell_{k}}$ for some constant $c$. For the second term, then, we have

$$
\#\left(S_{k} R^{-1}\right) \leq \#\left(\mathbb{A}^{c \ell_{k}} R^{-1}\right) \leq C \#\left(S(k, r) S^{-1}(k, r)\right) .
$$

In a similar way one may show that the third term is less than $C \#\left(S(k, r) S^{-1}(k, r)\right)$. 
5.2. Random averages for measure-preserving group actions. Let $\Omega$ be a probability space, let $0<\alpha<d$, and let $\left\{\xi_{g}(\omega): g \in G\right\}$ be independent $\{0,1\}$ valued random variables on $\Omega$ with $\mathbb{P}\left(\xi_{g}=1\right)=\rho(g, e)^{-\alpha}$. Note that by Theorem 5.1 and the Strong Law of Large Numbers, there exists $C$ depending on $G, \mathbb{A}$ and $\alpha$ such that $\mathbb{P}\left(N^{\alpha-d} \sum_{g \in \mathbb{A}^{N}} \xi_{g} \rightarrow C\right)=1$. We restrict ourselves to this set $\Omega_{1}$ of probability 1 .

Definition. For a measure-preserving group action $\left(X, \mathcal{F}, m,\left\{T_{g}\right\}\right)$ and $f \in L^{1}(X)$, define the average

$$
A_{N} f(x):=\frac{1}{\# \mathbb{A}^{N}} \sum_{g \in \mathbb{A}^{N}} f\left(T_{g} x\right)
$$

and the random average

$$
A_{N}^{(\omega)} f(x):=N^{\alpha-d} \sum_{g \in \mathbb{A}^{N}} \xi_{g}(\omega) f\left(T_{g} x\right) .
$$

Krengel proves several theorems about measure-preserving group actions and other additive processes in Section 6.4 of [14]. We will apply Theorems 4.1, 4.2, and 4.4 from that section to our particular case:

Theorem 5.3. Let $G$ have polynomial growth of degree $d$, and $\mathbb{A}$ be a finite symmetric generating set. Then for every measure-preserving group action $\left(X, \mathcal{F}, m,\left\{T_{g}\right\}\right)$ and $1 \leq p<\infty, A_{N} f$ converges in $L^{p}$ and a.e. for every $f \in L^{p}(X, m)$.

Theorem 5.4. Let $G$ have polynomial growth of degree $d$, and $\mathbb{A}$ be a finite symmetric generating set. Then we have a weak-type maximal inequality on $G$ itself,

$$
\#\left\{g \in G: \sup _{N}\left|\varphi * \frac{1}{\# \mathbb{A}^{N}} \mathbf{1}_{\mathbb{A}^{N}}\right|>\lambda\right\} \leq \frac{C}{\lambda}\|\varphi\|_{1} \quad \text { for all } \varphi \in \ell^{1}(G) .
$$

We may now state our main results:

Theorem 5.5. Let $G$ be a finitely generated group with polynomial growth of degree $d$, and $\mathbb{A}$ a finite symmetric generating set, and $0<\alpha<d$. Then there exists $\Omega_{2} \subset \Omega$ with $\mathbb{P}\left(\Omega_{2}\right)=1$ such that for each $\omega \in \Omega_{2}, A_{N}^{(\omega)} f$ converges in $L^{2}$ and a.e. for every measure-preserving group action $\left(X, \mathcal{F}, m,\left\{T_{g}\right\}\right)$ and every $f \in L^{2}(X, m)$.

Theorem 5.6. Let $G$ be a finitely generated group with polynomial growth of degree $d$, and $\mathbb{A}$ a finite symmetric generating set, and $0<\alpha<d / 2$. Then there exists $\Omega_{3} \subset \Omega$ with $\mathbb{P}\left(\Omega_{3}\right)=1$ such that for each $\omega \in \Omega_{3}, A_{N}^{(\omega)} f$ converges in $L^{1}$ and a.e. for every measure-preserving group action $\left(X, \mathcal{F}, m,\left\{T_{g}\right\}\right)$ and every $f \in L^{1}(X, m)$.

5.2.1. Proof of Theorem 5.5. The analogue of Theorem 5.5 was proved by Bourgain [5] using the theory of exponential sums, and this technique extends to the natural analogues in $\mathbb{Z}^{d}$. However, on virtually nilpotent groups the Fourier transform is not so easy to work with, and so we will prove the $L^{2}$ theorem using the $T T^{*}$ method and a lemma from combinatorics.

It will suffice to prove the convergence of $A_{N}^{(\omega)} f$ along a suitable subsequence. Indeed, fix an increasing sequence $\left\{N_{j}\right\} \subset \mathbb{N}$ such that $\frac{N_{j+1}}{N_{j}} \rightarrow 1$. Then for any $f \geq 0$ and $N_{j} \leq N \leq N_{j+1}$,

$$
\left(\frac{N_{j}}{N_{j+1}}\right)^{d-\alpha} A_{N_{j}}^{(\omega)} f \leq A_{N}^{(\omega)} f \leq\left(\frac{N_{j+1}}{N_{j}}\right)^{d-\alpha} A_{N_{j+1}}^{(\omega)} f .
$$


Then under the assumptions of Theorem [5.5, it suffices to prove that $A_{N_{j}}^{(\omega)} f$ converges in $L^{2}$ and a.e. for all $f \in L^{2}(X)$. We may assume that $\left\{N_{j}\right\}$ is superpolynomial; i.e. $N_{j} \gg j^{C}$ for every $C \in \mathbb{N}$.

We will compare these random averages to their expected value, which is a weighted average of the standard ergodic averages. Define

$$
\sigma_{N} f(x):=\mathbb{E}_{\omega} A_{N}^{(\omega)} f(x)=N^{\alpha-d} \sum_{g \in \mathbb{A}^{N}} \rho(g, e)^{-\alpha} f\left(T_{g} x\right)=\sum_{n=0}^{N} a_{n, N} A_{n} f(x),
$$

where $a_{n, N} \geq 0, \sum_{n=0}^{N} a_{n, N}=1$ for all $N$, and $\lim _{N \rightarrow \infty} a_{n, N}=0$ for all $n$. Since $A_{n} f$ converges in $L^{2}$ and a.e. by Theorem 5.3 , clearly $\sigma_{N} f$ converges in $L^{2}$ and a.e. as well.

We will prove Theorem 5.5 by showing that there exists a set $\Omega_{2} \subset \Omega_{1}$ with $\mathbb{P}\left(\Omega_{2}\right)=1$ such that for every $\omega \in \Omega_{2}$,

$$
\left\|\sup _{j \geq k}\left|A_{N_{j}}^{(\omega)} f-\sigma_{N_{j}} f\right|\right\|_{2} \rightarrow 0 \text { as } k \rightarrow \infty \text { for all } f \in L^{2}(X),
$$

which immediately implies $A_{N_{j}}^{(\omega)} f-\sigma_{N_{j}} f \rightarrow 0$ in $L^{2}$ and a.e.

As in [4] and other papers, we hope to transfer the corresponding maximal inequality from the group algebra $\ell^{p}(G)$. This Calderón transference principle is practically identical to the case $G=\mathbb{Z}$, but it is necessary to prove it in this general setting.

Lemma 5.7. Let $G$ be a group with polynomial growth, and $\left(X, \mathcal{F}, m,\left\{T_{g}\right\}\right)$ be a measure-preserving group action; let $\left\{a_{g, j}\right\} \subset \mathbb{C}$ such that $\sum_{g \in G}\left|a_{g, j}\right|<\infty$ for all $j$. Set $A_{j} f=\sum_{g \in G} a_{g, j} T_{g} f$ and $\mu_{j}=\sum_{g \in G} a_{g, j} \delta_{g}$.

For any $1 \leq p \leq \infty$, if $\left\|\sup _{j}\left|\psi * \mu_{j}\right|\right\|_{p} \leq C_{0}\|\psi\|_{p}$ for all $\psi \in \ell^{p}(G)$, then $\left\|\sup _{j}\left|A_{j} f\right|\right\|_{p} \leq C_{0}\|f\|_{p}$ for all $f \in L^{p}(X)$;

if instead $\left\|\sup _{j}\left|\psi * \mu_{j}\right|\right\|_{p, \infty} \leq C_{0}\|\psi\|_{p}$ for all $\psi \in \ell^{p}(G)$, then $\left\|\sup _{j}\left|A_{j} f\right|\right\|_{p, \infty} \leq$ $C_{0}\|f\|_{p}$ for all $f \in L^{p}(X)$.

Proof. We first consider the strong maximal inequality. It is enough to show that $\left\|\sup _{1 \leq j \leq J}\left|A_{j} f\right|\right\|_{p} \leq C_{0}\|f\|_{p}$ for all $f \in L^{p}(X)$, for each fixed $J \in \mathbb{N}$. We may further assume that the supports of the $\mu_{j}$ are finite, and let $\mathcal{E}:=\bigcup_{j=1}^{J}$ supp $\mu_{j}$. Take a finite symmetric set $\mathbb{A}$ that generates $G$, and the sets $\mathbb{A}^{N}$ defined in Section 5.2. Fix $x \in X$ and a large finite $K \in \mathbb{N}$, and define $\varphi$ on $G$ by

$$
\varphi(g)= \begin{cases}f\left(T_{g^{-1}} x\right) & \text { if } g^{-1} \in \mathbb{A}^{K}+\mathcal{E} \\ 0 & \text { otherwise. }\end{cases}
$$

Then $A_{j} f\left(T_{g} x\right)=\varphi * \mu_{j}\left(g^{-1}\right)$ for all $g \in \mathbb{A}^{K}$ and all $j \leq J$. This completes the proof for $p=\infty$; for $p<\infty$,

$$
\begin{aligned}
\sum_{g \in \mathbb{A}^{K}} \sup _{1 \leq j \leq J}\left|A_{j} f\left(T_{g} x\right)\right|^{p} & =\sum_{g \in \mathbb{A}^{K}} \sup _{1 \leq j \leq J}\left|\varphi * \mu_{j}\left(g^{-1}\right)\right|_{p} \leq\left\|\sup _{k \leq j \leq J}\left|\varphi * \mu_{j}\right|\right\|_{p}^{p} \\
& \leq C_{0}^{p}\|\varphi\|_{p}^{p} \\
& =C_{0}^{p} \sum_{g \in \mathbb{A}^{K}+\mathcal{E}}\left|f\left(T_{g} x\right)\right|^{p} .
\end{aligned}
$$


Integrating over $x \in X$,

$$
\left\|\sup _{1 \leq j \leq J}\left|A_{j} f\right|\right\|_{p}^{p} \leq C_{0}^{p} \frac{\#\left(\mathbb{A}^{K}+\mathcal{E}\right)}{\# \mathbb{A}^{K}}\|f\|_{p}^{p}
$$

we let $K \rightarrow \infty$ and note that (5.2) implies (with $C_{0}$ independent of $J$ )

$$
\left\|\sup _{1 \leq j \leq J}\left|A_{j} f\right|\right\|_{p} \leq C_{0}\|f\|_{p} .
$$

For the weak inequality, we similarly derive

$$
\lambda^{p} \#\left\{g \in \mathbb{A}^{K}: \sup _{1 \leq j \leq J}\left|A_{j} f\left(T_{g} x\right)\right|>\lambda\right\} \leq C_{0}^{p}\|\varphi\|_{p}^{p}
$$

and integrate this in the same manner.

Proof of Theorem 5.5 (Continued). We will transfer this problem to $\ell^{2}(G)$ using Lemma 5.7. Let $\eta_{g}(\omega)=\xi_{g}(\omega)-\rho(g, e)^{-\alpha}$; these are independent mean 0 Bernoulli variables. Define for each $j$ the random measures

$$
\nu_{j}^{(\omega)}(g)= \begin{cases}N_{j}^{\alpha-d} \eta_{g}(\omega), & g \in \mathbb{A}^{N_{j}} \\ 0, & g \notin \mathbb{A}^{N_{j}}\end{cases}
$$

Then for $\varphi \in \ell^{p}(G)$, we have the random averages

$$
\varphi * \nu_{j}^{(\omega)}(h)=N_{j}^{\alpha-d} \sum_{g \in \mathbb{A}^{N_{j}}} \xi_{g}(\omega) \varphi\left(h g^{-1}\right),
$$

which correspond to the operators $A_{N_{j}}^{(\omega)}-\sigma_{N_{j}}$ in the sense above. Theorem 5.5 therefore reduces to verifying that with probability 1 in $\Omega$, there is a sequence $C_{k, \omega} \rightarrow 0$ such that

$$
\left\|\sup _{j \geq k}\left|\psi * \nu_{j}^{(\omega)}\right|\right\|_{2} \leq C_{k, \omega}\|\psi\|_{2} \text { for all } \psi \in \ell^{2}(G) .
$$

Since $\left\|\sup _{j \geq k}\left|\psi * \nu_{j}^{(\omega)}\right|\right\|_{2}^{2} \leq\left\|\sum_{j \geq k}\left|\psi * \nu_{j}^{(\omega)}\right|\right\|_{2}^{2}=\sum_{j \geq k}\left\|\psi * \nu_{j}^{(\omega)}\right\|_{2}^{2}$, it clearly suffices to prove that

$$
\sum_{j=1}^{\infty}\left\|\nu_{j}^{(\omega)}\right\|_{o p}^{2} \leq \infty
$$

where $\|\cdot\|_{o p}$ is the norm of the convolution operator on $\ell^{2}(G)$.

Since in this context we do not have the Fourier transform to help us, we will use a different Hilbert space technique: the $T T^{*}$ method from harmonic analysis.

For any operator $A$ on the Hilbert space $\ell^{2}(G)$, the operator norm $\|A\|=$ $\left\|A^{*} A\right\|^{1 / 2}=\left\|\left(A^{*} A\right)^{M}\right\|^{1 / 2 M}$; for the convolution operator $A f=\mu * f$, the adjoint operator is simply $A^{*} f=\tilde{\mu} * f$ for $\tilde{\mu}(g):=\overline{\mu\left(g^{-1}\right)}(G$ is discrete, thus unimodular). Thus we have the trivial bound $\|A\|_{o p} \leq\left\|(\tilde{\mu} * \mu)^{M}\right\|_{o p}^{1 / 2 M} \leq\left\|(\tilde{\mu} * \mu)^{M}\right\|_{\ell^{1}}^{1 / 2 M}$, and any cancellation in the convolution products will make itself known in the original operator norm. (Here and in what follows, we use $\mu^{n}$ to denote the $n$-fold convolution product $\mu * \mu * \cdots * \mu$.)

The cancellation in this convolution product can be described in terms of additive combinatorics on $G$ : if we take a random subset $E \subset \mathbb{A}^{N}$ with size 》 $\left(\# \mathbb{A}^{N}\right)^{1 / 2 M}$, then the number of ways to write any element of $\mathbb{A}^{2 M N}$ as a product $g_{1} g_{2}^{-1} \ldots g_{2 M-1} g_{2 M}^{-1}$ with all $g_{i} \in E$ should most of the time be quite close to the "average" number of ways to do so. The quantitative version of this is as follows: 
Lemma 5.8. Let $G$ be a group and $E$ a finite subset. Let $\left\{X_{g}\right\}_{g \in E}$ be independent random variables with $\left|X_{g}\right| \leq 1$ and $\mathbb{E} X_{g}=0$. Assume that $\sum_{g \in E} \operatorname{Var} X_{g} \geq$ 1. Let $X$ be the random $\ell^{1}(G)$ function $\sum_{g \in E} X_{g} \delta_{g}$. Then $\mathbb{E}\left\|(\tilde{X} * X)^{M}\right\|_{\ell^{2}}^{2} \leq$ $C_{M}\left(\sum_{g \in E} \operatorname{Var} X_{g}\right)^{2 M}$, where $C_{M}$ depends only on $M$.

Proof.

$$
\begin{aligned}
& \mathbb{E}\left(\left\|(\tilde{X} * X)^{M}\right\|_{\ell^{2}}^{2}\right) \\
& =\mathbb{E} \sum_{g \in G}\left[\sum_{\substack{\ldots g_{M} h_{M}^{-1}=g \\
g_{1} h_{i}^{-1} \in E}} X_{g_{1}} X_{h_{1}} \ldots X_{g_{M}} X_{h_{M}}\right]^{2} \\
& =\sum_{\substack{g_{1} h_{1}^{-1} \ldots g_{M} h_{M}^{-1}=g_{M+1} h_{M+1}^{-1} \ldots g_{2 M} h_{2 M}^{-1} \\
g_{i}, h_{i} \in E}} \mathbb{E}\left(X_{g_{1}} X_{h_{1}} \ldots X_{g_{2 M}} X_{h_{2 M}}\right) .
\end{aligned}
$$

For any of these terms, if some $g \in E$ appears exactly once among $g_{i}$ and $h_{j}$, the expectation of the term will equal 0 by the independence of $X_{g}$. Therefore we can sort the remaining terms based on the equalities between various $g_{i}$ and $h_{j}$, namely, in correspondence with the set partitions of $\{1, \ldots, 4 M\}$ in which each component has size $\geq 2$. Let there be $C_{M}$ of these. For a fixed partition $\Lambda=\left(\lambda_{1}, \ldots, \lambda_{q}\right)$, we can majorize the sum

$$
\begin{aligned}
& \sum_{\left(g_{1}, \ldots, g_{2 M}, h_{1}, \ldots, h_{2 M}\right) \in \Lambda} \mathbb{E}\left(X_{g_{1}} \ldots X_{g_{2 M}} X_{h_{1}} \ldots X_{h_{2 M}}\right) \\
\leq & \sum_{g_{1}, \ldots, g_{q} \in E \text { distinct }} \mathbb{E}\left(\left|X_{g_{1}}\right|^{\left|\lambda_{1}\right|}\right) \ldots \mathbb{E}\left(\left|X_{g_{q}}\right|^{\left|\lambda_{q}\right|}\right) \\
\leq & \sum_{g_{1}, \ldots, g_{q} \in E} \mathbb{E} X_{g_{1}}^{2} \ldots \mathbb{E} X_{g_{q}}^{2} \\
= & \left(\sum_{g \in E} \operatorname{Var} X_{g}\right)^{q} \leq\left(\sum_{g \in E} \operatorname{Var} X_{g}\right)^{2 M}
\end{aligned}
$$

since $\mathbb{E}\left|X_{g}\right|^{p} \leq\left\|X_{g}\right\|_{\infty}^{p-2} \mathbb{E} X_{g}^{2} \leq \mathbb{E} X_{g}^{2}$ for $p>2, \sum_{g \in E} \operatorname{Var} X_{g} \geq 1$ and $q \leq 2 M$.

Thus $\mathbb{E}\left(\left\|(\tilde{X} * X)^{M}\right\|_{\ell^{2}}^{2}\right) \leq C_{M}\left(\sum_{g \in E} \operatorname{Var} X_{g}\right)^{2 M}$.

Proof of Theorem 5.5 (Conclusion). Now by Hölder's Inequality and the fact that $\left(\tilde{\nu}_{j} * \nu_{j}\right)^{M}$ is supported on $\mathbb{A}^{2 M N_{j}}$,

$$
\left\|\left(\tilde{\nu}_{j} * \nu_{j}\right)^{M}\right\|_{1} \leq\left\|\left(\tilde{\nu}_{j} * \nu_{j}\right)^{M}\right\|_{2}\left(\# \mathbb{A}^{2 M N_{j}}\right)^{1 / 2} \leq\left\|\left(\tilde{\nu}_{j} * \nu_{j}\right)^{M}\right\|_{2} C\left(2 M N_{j}\right)^{d / 2} .
$$

By Lemma 5.8, since $\operatorname{Var} \eta_{g} \leq \rho(g, e)^{\alpha}$,

$$
\mathbb{E}\left(\left\|\left(\tilde{\nu}_{j}^{(\omega)} * \nu_{j}^{(\omega)}\right)^{M}\right\|_{\ell^{2}}^{2}\right) \leq N_{j}^{4 M(\alpha-d)} \cdot C_{M}\left(\sum_{g \in \mathbb{A}^{N_{j}}} \operatorname{Var} \eta_{g}\right)^{2 M} \leq C_{d, \alpha, M} N_{j}^{2 M(\alpha-d)},
$$


and therefore by Chebyshev's Inequality,

$$
\begin{aligned}
\mathbb{P}\left(\left\|\left(\tilde{\nu}_{j}^{(\omega)} * \nu_{j}^{(\omega)}\right)^{M}\right\|_{1}>\lambda\right) & \leq \mathbb{P}\left(\left\|\left(\tilde{\nu}_{j}^{(\omega)} * \nu_{j}^{(\omega)}\right)^{M}\right\|_{2}^{2} C^{2}\left(2 M N_{j}\right)^{d}>\lambda^{2}\right) \\
& \leq C \lambda^{-2} M^{d} N_{j}^{d} \cdot \mathbb{E}\left(\left\|\left(\tilde{\nu}_{j}^{(\omega)} * \nu_{j}^{(\omega)}\right)^{M}\right\|_{\ell^{2}}^{2}\right) \\
& \leq C_{d, \alpha, M} \lambda^{-2} N_{j}^{2 M \alpha-d(2 M-1)} .
\end{aligned}
$$

As $\alpha<d$, take $M, \delta>0$ such that $d(2 M-1)>2 M \alpha+\delta$. Take $\lambda=j^{-M(1+\epsilon)}$; since $N_{j}^{\delta}$ is superpolynomial, $\sum_{j} j^{2 M(1+\epsilon)} N_{j}^{-\delta}<\infty$, so by the Borel-Cantelli Lemma, there is a set $\Omega_{2} \subset \Omega_{1}$ of probability 1 on which $\left\|\left(\tilde{\nu}_{j}^{(\omega)} * \nu_{j}^{(\omega)}\right)^{M}\right\|_{1}<C_{\omega} j^{-M(1+\epsilon)}$ for all $j$, and thus $\sum_{j=1}^{\infty}\left\|\nu_{j}^{(\omega)}\right\|_{o p}^{2} \leq C_{\omega} \sum_{j=1}^{\infty} j^{-1-\epsilon}<\infty$. This completes the proof of Theorem 5.5 .

5.2.2. Proof of Theorem 5.6. By Theorem 5.5, for $\omega \in \Omega_{2}$ we have a.e. convergence of $A_{N}^{(\omega)} f$ for $f \in L^{2}(X)$, which is dense in $L^{1}(X)$. We therefore need only a weak type maximal inequality to prove Theorem 5.6. As usual, it is enough to consider the dyadic subsequence $2^{j}$. Now for $f \geq 0,0 \leq A_{N}^{(\omega)} f \lesssim A_{2^{j+1}}^{(\omega)} f$ for $2^{j} \leq N<2^{j+1}$, so it suffices to prove

$$
\left\|\sup _{j}\left|A_{2^{j}}^{(\omega)} f\right|\right\|_{1, \infty} \leq C\|f\|_{1} \text { for all } f \in L^{1}(X) .
$$

Again, we will use Lemma 5.7 to transfer this maximal inequality from $\ell^{1}(G)$. Let

$$
\begin{aligned}
\mu_{j}^{(\omega)}(g) & := \begin{cases}2^{(\alpha-d) j} \xi_{g}(\omega), & g \in \mathbb{A}^{2^{j}}, \\
0, & g \notin \mathbb{A}^{2^{j}},\end{cases} \\
\mathbb{E} \mu_{j}(g) & := \begin{cases}2^{(\alpha-d) j} \mathbb{E} \xi_{g}, & g \in \mathbb{A}^{2^{j}}, \\
0, & g \notin \mathbb{A}^{2^{j}},\end{cases} \\
\nu_{j}^{(\omega)}(g) & :=\mu_{j}^{(\omega)}(g)-\mathbb{E} \mu_{j}^{(\omega)}(g) ;
\end{aligned}
$$

$\mu_{j}^{(\omega)}$ and $\mathbb{E} \mu_{j}$ correspond to the operators $A_{2^{j}}^{(\omega)}$ and $\sigma_{2^{j}}$, respectively. Theorem 5.6 reduces to proving

$$
\left\|\sup _{j}\left|\varphi * \mu_{j}^{(\omega)}\right|\right\|_{1, \infty} \leq C_{\omega}\|\varphi\|_{1} .
$$

Proposition 5.9. Let $\mu_{j}$ and $\nu_{j}$ be sequences of functions in $\ell^{1}(G)$, where $G$ has polynomial growth of degree d. Let $r_{j}:=\#\left\{g: \mu_{j}(g) \neq 0\right\}$ and take $R_{j}:=$ $\inf \left\{R>0: \nu_{j}(g) \neq 0 \Longrightarrow \rho(g, e) \leq R\right\}$. Assume there exists $C_{0}<\infty$ such that $\sum_{j \leq k} r_{j} \leq C_{0} r_{k}$ for all $k \in \mathbb{N}$, and that

$$
\nu_{j} * \tilde{\nu}_{j}=O\left(r_{j}^{-1}\right) \delta_{e}+O\left(R_{j}^{-d-\epsilon}\right) \text { for some } \epsilon>0 .
$$

If for all $\varphi,\left\|\sup \varphi *\left|\mu_{j}-\nu_{j}\right|\right\|_{1, \infty} \leq C\|\varphi\|_{1}$ and $\left\|\sup \left|\varphi * \mu_{j}\right|\right\|_{p, \infty} \leq C_{p}\|\varphi\|_{p}$ for some $1<p \leq \infty$, then

$$
\left\|\sup _{j}\left|\varphi * \mu_{j}\right|\right\|_{1, \infty} \leq C^{\prime}\|\varphi\|_{1} \text { for all } \varphi \in \ell^{1}(G) .
$$

Proof. This is simply an extension of the proof of Theorem 3.2 however, we must first establish that the Calderón-Zygmund decomposition makes sense on more general groups $G$. Since word-length is a quasimetric on $G$, we can use the $\rho$ dyadic cubes constructed by Christ in [9] on spaces of homogeneous type. Namely, 
there exists a collection of subsets $\left\{Q_{s, k} \subset G: s \in \mathbb{N}, k \in \mathbb{Z}\right\}$ and constants $A>1, a_{0}>0, C_{1}<\infty$ such that

$$
\begin{gathered}
\forall s \in \mathbb{N}, G=\bigcup_{k} Q_{s, k}, \\
r \leq s \Longrightarrow Q_{r, l} \subset Q_{s, k} \text { or } Q_{r, l} \cap Q_{s, k}=\emptyset, \\
\forall(r, l), \forall s>r, \exists ! k \in \mathbb{Z} \text { such that } Q_{r, l} \subset Q_{s, k}, \\
\text { diameter } Q_{s, k} \leq C_{1} A^{s}, \\
\text { each } Q_{s, k} \text { contains some ball of radius } a_{0} A^{s} .
\end{gathered}
$$

Because $G$ has a polynomial rate of growth, $\rho$ is a doubling metric, and thus we can prove the Vitali Covering Lemma and the Hardy-Littlewood Maximal Inequality on $G$. Using a standard stopping-time argument, we can then define a suitable discrete Calderón-Zygmund decomposition on $G$ with dyadic cubes.

Fix $\lambda>0$. We take $\varphi=\mathfrak{g}+b$, where $\|\mathfrak{g}\|_{\infty} \leq \lambda$ and $b=\sum_{(s, k) \in \mathcal{B}} b_{s, k}$ for some index set $\mathcal{B} \subset \mathbb{N}^{2}$, where $b_{s, k}$ is supported on $Q_{s, k},\left\{Q_{s, k}:(s, k) \in \mathcal{B}\right\}$ is a disjoint collection, $\left\|b_{s, k}\right\|_{1} \leq \lambda \# Q_{s, k}$ and $\sum_{(s, k) \in \mathcal{B}} \# Q_{s, k} \leq \frac{C}{\lambda}\|\varphi\|_{1}(C$ independent of $\varphi$ and $\lambda)$. Let $b_{s}=\sum_{k} b_{s, k}$.

We further decompose $b_{s, k}=b_{s, k}^{(j)}+B_{s, k}^{(j)}$, where $b_{s, k}^{(j)}=b_{s, k} \mathbf{1}\left(\left|b_{s, k}\right|>\lambda r_{j}\right)$. Define $b_{s}^{(j)}, B_{s}^{(j)}, b^{(j)}, B^{(j)}$ by summing over one or both indices, respectively.

We will divide $B^{(j)}=\sum_{s} B_{s}^{(j)}$ into two parts, splitting at the index $s(j):=$ $\min \left\{s: A^{s} \geq R_{j}\right\}$.

Now $\left\{g: \sup _{j}\left|\varphi * \mu_{j}(g)\right|>5 \lambda\right\} \subset E_{1} \cup E_{2} \cup E_{3} \cup E_{4} \cup E_{5}$, where

$$
\begin{aligned}
& E_{1}=\left\{g: \sup _{j}\left|\mathfrak{g} * \mu_{j}(g)\right|>\lambda\right\}, \\
& E_{2}=\left\{g: \sup _{j}\left|b^{(j)} * \mu_{j}(g)\right|>\lambda\right\}, \\
& E_{3}=\left\{g: \sup _{j}\left|B^{(j)} *\left(\mu_{j}-\nu_{j}\right)(g)\right|>\lambda\right\}, \\
& E_{4}=\left\{g: \sup _{j}\left|\left(\sum_{s=s(j)}^{\infty} B_{s}^{(j)}\right) * \nu_{j}(g)\right|>\lambda\right\}, \\
& E_{5}=\left\{g: \sup _{j}\left|\left(\sum_{s=0}^{s(j)-1} B_{s}^{(j)}\right) * \nu_{j}(g)\right|>\lambda\right\} .
\end{aligned}
$$

By the weak $(p, p)$ inequality (if $p<\infty), \# E_{1} \leq C \lambda^{-p}\|\mathfrak{g}\|_{p}^{p} \leq C \lambda^{-p}\|\mathfrak{g}\|_{\infty}^{p-1}\|\mathfrak{g}\|_{1} \leq$ $C \lambda^{-1}\|\varphi\|_{1}$; if $p=\infty$, re-do the decomposition so that $\|\mathfrak{g}\|_{\infty}<C_{\infty}^{-1} \lambda$ instead. Then $E_{1}$ will be empty since $\left\|\sup _{j}\left|\mathfrak{g} * \mu_{j}\right|\right\|_{\infty} \leq C_{\infty}\|\mathfrak{g}\|_{\infty} \leq \lambda$. 
Next,

$$
\begin{aligned}
\# E_{2} \leq \sum_{j} \#\left\{g:\left|b^{(j)} * \mu_{j}(g)\right|>0\right\} & \leq \sum_{j} \#\left(\operatorname{supp} \mu_{j}\right) \cdot \#\left\{g:|b(g)|>\lambda r_{j}\right\} \\
& =\sum_{j} r_{j} \sum_{k \geq j} \#\left\{g: \lambda r_{k}<|b(g)| \leq \lambda r_{k+1}\right\} \\
& =\sum_{k} \#\left\{g: \lambda r_{k}<|b(g)| \leq \lambda r_{k+1}\right\} \sum_{j \leq k} r_{j} \\
& \leq \frac{C_{0}}{\lambda} \sum_{k} \lambda r_{k} \#\left\{g: \lambda r_{k}<|b(g)| \leq \lambda r_{k+1}\right\}
\end{aligned}
$$

now note that this sum is a lower sum for $|b|$, and we have $\# E_{2} \leq C_{0} \lambda^{-1}\|b\|_{1} \leq$ $\frac{C}{\lambda}\|\varphi\|_{1}$.

For $E_{3},\left|B^{(j)} *\left(\mu_{j}-\nu_{j}\right)(g)\right| \leq\left|B^{(j)}\right| *\left|\mu_{j}-\nu_{j}\right|(g) \leq|b| *\left|\mu_{j}-\nu_{j}\right|(g)$, so by the weak- $(1,1)$ inequality,

$$
\# E_{3} \leq \#\left\{g: \sup _{j}|b| *\left|\mu_{j}-\nu_{j}\right|(g)>\lambda\right\} \leq \frac{C}{\lambda}\|b\|_{1} \leq \frac{C}{\lambda}\|\varphi\|_{1} .
$$

To bound $E_{4}$, note that for all $s \geq s(j), B_{s, k}^{(j)} * \nu_{j}$ is supported on $Q_{s, k}^{*}:=\{g$ : $\left.\rho\left(g, Q_{s, k}\right) \leq A^{s}\right\}$, so

$$
\# E_{4} \leq \sum_{(s, k) \in \mathcal{B}} C \# Q_{s, k} \leq \frac{C}{\lambda}\|\varphi\|_{1} .
$$

We have thus reduced the problem to obtaining a bound on the size of $E_{5}$.

Lemma 5.10. Let $B_{s}^{(j)}$ be as above, and assume $\nu_{j}$ satisfy (5.11). For $0 \leq s<s(j)$,

$$
\left\|B_{s}^{(j)} * \nu_{j}\right\|_{\ell^{2}(G)}^{2} \leq C r_{j}^{-1}\left\|B_{s}^{(j)}\right\|_{2}^{2}+C \lambda 2^{-\epsilon j}\left\|B_{s}^{(j)}\right\|_{1},
$$

and for $0 \leq s_{1}<s_{2}<s(j)$,

$$
\left|\left\langle B_{s_{1}}^{(j)} * \nu_{j}, B_{s_{2}}^{(j)} * \nu_{j}\right\rangle_{\ell^{2}(G)}\right| \leq C \lambda 2^{-\epsilon j}\left\|B_{s_{2}}^{(j)}\right\|_{1} .
$$

Proof. We first restrict the supports of $B_{s}$; we assume there is a $Q_{s(j), k_{0}}$ such that $Q_{s, k} \subset Q_{s(j), k_{0}}$ for all $(s, k) \in \mathcal{B}$ with $s<s(j)$. Then $\left\|B_{s}^{(j)}\right\|_{1} \leq\left\|b_{s}\right\|_{1} \leq$ $\sum_{(s, k) \in \mathcal{B}} \lambda\left|Q_{s, k}\right| \leq \lambda\left|Q_{s(j), k_{0}}\right| \leq C \lambda R_{j}^{d}$, and thus

$$
\begin{aligned}
\left|\left\langle B_{s_{1}}^{(j)} * \nu_{j}, B_{s_{2}}^{(j)} * \nu_{j}\right\rangle\right| & =\left|\left\langle B_{s_{1}}^{(j)} * \nu_{j} * \tilde{\nu}_{j}, B_{s_{2}}^{(j)}\right\rangle\right| \\
& \leq C r_{j}^{-1}\left|\left\langle B_{s_{1}}^{(j)}, B_{s_{2}}^{(j)}\right\rangle\right|+C R_{j}^{-d} 2^{-\epsilon j}\left\|B_{s_{1}}^{(j)}\right\|_{1}\left\|B_{s_{2}}^{(j)}\right\|_{1} \\
& \leq C r_{j}^{-1}\left|\left\langle B_{s_{1}}^{(j)}, B_{s_{2}}^{(j)}\right\rangle\right|+C \lambda 2^{-\epsilon j}\left\|B_{s_{2}}^{(j)}\right\|_{1} .
\end{aligned}
$$

Now this first term is 0 if $s_{1} \neq s_{2}$, and $C r_{j}^{-1}\left\|B_{s_{1}}^{(j)}\right\|_{2}^{2}$ if $s_{1}=s_{2}$.

We remove the assumption on the supports by noting that if the distance between the supports of $\varphi_{1}$ and $\varphi_{2}$ is greater than $2 R_{j}$, then $\left\langle\varphi_{1} * \nu_{j}, \varphi_{2} * \tilde{\nu}_{j}\right\rangle=0$. Thus if we decompose each $B_{s}=\sum_{k} B_{s} \mathbf{1}\left(Q_{s(j), k}\right)$ and decompose the inner products accordingly, all but finitely many of the terms (a number independent of $j$ ) will vanish, and those remaining can be estimated in this way. 
Now by Chebyshev's Inequality,

$$
\begin{aligned}
\lambda^{2} \#\left\{g: \sup _{j}\left|\sum_{s=0}^{s(j)-1} B_{s}^{(j)} * \nu_{j}(g)\right|>\lambda\right\} & \leq \sum_{g} \sup _{j}\left|\sum_{s=0}^{s(j)-1} B_{s}^{(j)} * \nu_{j}(g)\right|^{2} \\
& \leq \sum_{j}\left\|\sum_{s=0}^{s(j)-1} B_{s}^{(j)} * \nu_{j}\right\|_{2}^{2} \\
& \leq \sum_{j} \sum_{\substack{s_{1}, s_{2}: \\
0 \leq s_{1}, s_{2}<s(j)}}\left|\left\langle B_{s_{1}}^{(j)} * \nu_{j}, B_{s_{2}}^{(j)} * \nu_{j}\right\rangle_{\ell^{2}(G)}\right|,
\end{aligned}
$$

and this is

$$
\begin{aligned}
\leq & \sum_{j} \sum_{s=0}^{s(j)-1}\left(C r_{j}^{-1}\left\|B_{s}^{(j)}\right\|_{2}^{2}+C \lambda 2^{-\epsilon j}\left\|B_{s}^{(j)}\right\|_{1}\right) \\
& +2 \sum_{j} \sum_{\substack{s_{1}, s_{2}: \\
0 \leq s_{1}<s_{2}<s(j)}} C \lambda 2^{-\epsilon j}\left\|B_{s_{2}}^{(j)}\right\|_{1} \\
\leq & \sum_{s=0}^{\infty} \sum_{j=1}^{\infty} C \lambda(1+j) 2^{-\epsilon j}\left\|B_{s}^{(j)}\right\|_{1}+\sum_{j} \sum_{s=0}^{s(j)-1} C r_{j}^{-1}\left\|B_{s}^{(j)}\right\|_{2}^{2} \\
\leq & \sum_{s=0}^{\infty} C \lambda\left\|b_{s}\right\|_{1}+\sum_{j} \sum_{s=0}^{s(j)-1} C r_{j}^{-1}\left\|B_{s}^{(j)}\right\|_{2}^{2} .
\end{aligned}
$$

The first term is $\leq C \lambda\|\varphi\|_{1}$, as desired. For the second term, note that

$$
\begin{aligned}
\sum_{j \leq k} r_{j} \leq C_{0} r_{k} \text { for all } k \in \mathbb{N} \Longrightarrow \exists N \\
\text { s.t. } r_{j+n} \geq 2 r_{j} \text { for all } j \in \mathbb{N}, n \geq N \Longrightarrow \sum_{j=k}^{\infty} r_{j}^{-1} \leq C r_{k}^{-1} .
\end{aligned}
$$

Since the $Q_{s, k}$ are disjoint, for a fixed $g \in Q_{s_{0}, k_{0}}$,

$$
\sum_{j} \sum_{s=0}^{s(j)-1} r_{j}^{-1}\left|B_{s}^{(j)}(g)\right|^{2} \leq \sum_{\substack{j: \\ \lambda r_{j} \geq\left|b_{s_{0}}(g)\right|}} r_{j}^{-1}\left|b_{s_{0}}(g)\right|^{2} \leq C \lambda\left|b_{s_{0}}(g)\right|=C \lambda|b(g)|,
$$

so $\sum_{j} \sum_{s=0}^{j-1} C r^{-j}\left\|B_{s}^{(j)}\right\|_{2}^{2} \leq C \lambda\|b\|_{1} \leq C \lambda\|\varphi\|_{1}$ and the proof of (5.12) is complete.

Having established Proposition 5.9, it remains to show that the random measures $\mu_{j}^{(\omega)}$ and $\nu_{j}^{(\omega)}$ satisfy the assumptions with probability 1 . Note first that $r_{j}=$ $\left|\operatorname{supp} \mu_{j}^{(\omega)}\right|=\sum_{g \in \mathbb{A}^{2 j}} \xi_{g}(\omega) \lesssim 2^{(d-\alpha) j}$ on $\Omega_{1}$, and $\nu_{j}^{(\omega)}$ is supported on $\mathbb{A}^{2^{j}}$ with $\rho$-diameter at most $R_{j}=2^{j+1}$. We must prove the bound (5.11) on $\nu_{j}^{(\omega)} * \tilde{\nu}_{j}^{(\omega)}$.

Lemma 5.11. Let $G$ be a group and $E$ a finite subset. Let $\left\{X_{g}\right\}_{g \in E}$ be independent random variables with $\left|X_{g}\right| \leq 1$ and $\mathbb{E} X_{g}=0$. Assume that $\sum_{g \in E}\left(\operatorname{Var} X_{g}\right)^{2} \geq 1$. 
Let $X$ be the random $\ell^{1}(G)$ function $\sum_{g \in E} X_{g} \delta_{g}$. Let $G^{\times}$denote $G \backslash\{e\}$. Then for any $\theta>0$,

$$
\mathbb{P}\left(\|X * \tilde{X}\|_{\ell^{\infty}\left(G^{\times}\right)} \geq \theta\left(\sum_{g \in E}\left(\operatorname{Var} X_{g}\right)^{2}\right)^{1 / 2}\right) \leq 6|E|^{2} \max \left(e^{-\theta^{2} / 36}, e^{-\theta / 6}\right) .
$$

Proof. For $h \neq e$,

$$
X * \tilde{X}(h)=\sum_{g \in E \cap h^{-1} E} X_{g} X_{g h}=\sum_{g \in E \cap h^{-1} E} Y_{g},
$$

where $\mathbb{E} Y_{g}=0$ and $\left|Y_{g}\right| \leq 1$. We want to apply Chernoff's Inequality, but $Y_{g}$ are not independent.

We can, however, partition $E \cap h^{-1} E$ into at most three subsets $E_{1}, E_{2}, E_{3}$, in each of which the $Y_{g}$ are independent. To see this, note that we can make a directed graph with vertex set $E$ and edge set $\{(g, h g): g, h g \in E\}$, and that the components of this graph are paths or cycles. Thus we can three-color this graph; and within each resulting $E_{i}$, the $Y_{g}$ depend on distinct independent random variables, so they are independent.

Now $\sum_{g \in E_{i}} Y_{g}$ has variance

$$
\sigma^{2}=\sum_{g \in E_{i}} \operatorname{Var} X_{g} \operatorname{Var} X_{g h} \leq \sum_{g \in E_{i}}\left(\operatorname{Var} X_{g}\right)^{2} \leq \sum_{g \in E}\left(\operatorname{Var} X_{g}\right)^{2}
$$

by Hölder's Inequality. Chernoff's Inequality (Theorem 1.8 in 21]) gives us

$$
\mathbb{P}\left(\left|\sum_{g \in E_{i}} Y_{g}\right| \geq \lambda \sigma\right) \leq 2 \max \left(e^{-\lambda^{2} / 4}, e^{-\lambda \sigma / 2}\right) .
$$

Take $\lambda=\theta \sigma^{-1}\left(\sum_{g \in E}\left(\operatorname{Var} X_{g}\right)^{2}\right)^{1 / 2} ;$ then $\lambda \geq \theta$ and $\lambda \sigma=\theta\left(\sum_{g \in E}\left(\operatorname{Var} X_{g}\right)^{2}\right)^{1 / 2} \geq$ $\theta$, so

$$
\begin{aligned}
\mathbb{P}\left(|X * \tilde{X}(h)| \geq 3 \theta\left(\sum_{g \in E}\left(\operatorname{Var} X_{g}\right)^{2}\right)^{1 / 2}\right) & \leq \sum_{i=1}^{3} \mathbb{P}\left(\left|\sum_{E_{i}} Y_{g}\right| \geq \lambda \sigma\right) \\
& \leq 6 \max \left(e^{-\theta^{2} / 4}, e^{-\theta / 2}\right) .
\end{aligned}
$$

Since this holds for each $h \neq e$ and $|\operatorname{supp} X * \tilde{X}| \leq|E|^{2}$, the conclusion follows (after replacing $3 \theta$ with $\theta$ ).

Corollary 5.12. Let $\nu_{j}^{(\omega)}$ be the random measure defined as before, $0<\alpha<d / 2$ and $\kappa>0$. Then there is a set $\Omega_{3} \subset \Omega_{2}$ with $\mathbb{P}\left(\Omega_{3}=1\right)$ such that for each $\omega \in \Omega_{3}$,

$$
\nu_{j}^{(\omega)} * \tilde{\nu}_{j}^{(\omega)}=O_{\omega}\left(2^{(\alpha-d) j}\right) \delta_{e}+O_{\omega}\left(2^{2(\alpha-d) j}\left(\sum_{g \in \mathbb{A}^{2 j}} \mathbb{E} \xi_{g}^{2}\right)^{1 / 2} 2^{\kappa j}\right) .
$$

Proof. For the bound at the identity $e$, we use the fact that

$$
\begin{aligned}
\nu_{j}^{(\omega)} * \tilde{\nu}_{j}^{(\omega)}(e) & =2^{2(\alpha-d) j} \sum_{g \in \mathbb{A}^{2 j}} \eta_{g}^{2}(\omega) \\
& \leq 2^{2(\alpha-d) j} \sum_{g \in \mathbb{A}^{2 j}}\left(\mathbb{E} \xi_{g}+\xi_{g}(\omega)\right)=2^{(\alpha-d) j+1}+2^{2(\alpha-d) j} \sum_{g \in \mathbb{A}^{2 j}} \eta_{g}(\omega)
\end{aligned}
$$


so that

$\mathbb{P}\left(\nu_{j}^{(\omega)} * \tilde{\nu}_{j}^{(\omega)}(e)>3 \cdot 2^{(\alpha-d) j}\right) \leq \mathbb{P}\left(\sum_{g \in \mathbb{A}^{2^{j}}} \eta_{g}(\omega)>2^{(d-\alpha) j}\right) \leq 2 \exp \left(-\frac{1}{2} 2^{(d-\alpha) j}\right)$

for $j$ sufficiently large, by Chernoff's Inequality. The Borel-Cantelli Lemma then implies that $\nu_{j}^{(\omega)} * \tilde{\nu}_{j}^{(\omega)}(e) \leq 3 \cdot 2^{(\alpha-d) j}$ for $j$ sufficiently large (depending on $\omega$ ), so there exists $C_{\omega}$ with $0 \leq \nu_{j}^{(\omega)} * \tilde{\nu}_{j}^{(\omega)}(e) \leq C_{\omega} 2^{(\alpha-d) j}$ for all $j$.

For the other term, we note that $\operatorname{Var} \eta_{g} \leq \mathbb{E} \xi_{g}$, so we set $\theta=2^{\kappa j}$ and apply Lemma 5.11

$$
\mathbb{P}\left(2^{2(d-\alpha) j}\left\|\nu_{j}^{(\omega)} * \tilde{\nu}_{j}^{(\omega)}\right\|_{\ell^{\infty}\left(G^{\times}\right)} \geq 2^{\kappa j}\left(\sum_{g \in \mathbb{A}^{2}} \mathbb{E} \xi_{g}^{2}\right)^{1 / 2}\right) \leq C 2^{2 d j} \exp \left(-2^{\kappa j} / 2\right),
$$

which sum over $j$. The Borel-Cantelli Lemma again proves that the bound holds with probability 1 .

Note that $\sum_{g \in \mathbb{A}^{2}} \mathbb{E} \xi_{g}^{2} \lesssim 2^{(d-2 \alpha) j} ;$ thus for $\alpha<d / 2$,

$$
2^{2(\alpha-d) j}\left(\sum_{g \in \mathbb{A}^{2 j}} \mathbb{E} \xi_{g}^{2}\right)^{1 / 2} 2^{\kappa j} \leq C 2^{\left(-\frac{3 d}{2}+\alpha+\kappa\right) j}=C R_{j}^{-d} 2^{\left(-\frac{d}{2}+\alpha+\kappa\right) j},
$$

and thus for $\kappa$ chosen small, the measures $\nu_{j}^{(\omega)}$ satisfy the bound (5.11) for all $\omega \in \Omega_{3}$. Since $\mu_{j}^{(\omega)}-\nu_{j}^{(\omega)}=\mathbb{E} \mu_{j}$ is a weighted average of the nonnegative averages in (5.4), Theorem 5.4 implies $\left\|\sup _{j}\left|\varphi * \mathbb{E} \mu_{j}\right|\right\|_{1, \infty} \leq C\|\varphi\|_{1}$, and the $\ell^{\infty}$ maximal inequality for $\mu_{j}^{(\omega)}$ is trivial. Thus Proposition 5.9 applies, and we have proved Theorem 5.6 .

Remark. As in the case of $\mathbb{Z}^{d}$, this method is inherently limited to exponents $\alpha<$ $d / 2$, because otherwise the set is too sparse for a single convolution product to be "uniformly" small in any nontrivial sense.

5.3. Gaps and Banach density. In [15] it was noted that, with probability 1, the sparse random sequences in $\mathbb{N}$ have Banach density 0 , which distinguishes them from block sequences of the Bellow-Losert type in Section 2 This remains true for the random subset $\left\{g \in G: \xi_{g}(\omega)=1\right\}$ which we have obtained.

It is worth noting a second distinction: with probability 1 , this random set has a subset with gaps tending to infinity which is of full relative measure, and thus the averages over this subset still converge a.e. for functions in $L^{1}$. (Note that this is not a necessary consequence of Banach density 0: consider the "Cantor set" of natural numbers that can be written as a finite sum of distinct powers of 3 . This set has Banach density 0, but no set of positive relative measure can have gaps tending to $\infty$.)

We will order the random set $\left\{g_{n}\right\}=\left\{g \in G: \xi_{g}(\omega)=1\right\}$ so that $\rho\left(g_{n}, e\right)$ is nondecreasing. The convergence of the ergodic averages $A_{N_{j}}^{(\omega)}$ in (5.5) implies that we can add the points one at a time and maintain the pointwise ergodic theorem; 
that is, the averages

$$
A_{N}^{\left(g_{n}\right)} f(x):=\frac{1}{N} \sum_{n=1}^{N} f\left(\mathcal{T}\left(g_{n}\right) x\right)
$$

converge a.e. in $X$ for any measure-preserving group action $(X, \mathcal{T})$.

Definition. For $j \geq 0$ and $M<\infty$, let

$$
\begin{aligned}
\Gamma_{j, M} & :=\left\{n \in\left[2^{j}, 2^{j+1}\right): \inf _{m<n} \rho\left(g_{n}, g_{m}\right)<M\right\} \\
\beta_{t, M} & :=2^{-j}\left|\Gamma_{t, M}\right| .
\end{aligned}
$$

Proposition 5.13. Let $\left\{g_{n}\right\}$ be a sequence in a virtually nilpotent discrete group $G$, such that $\rho\left(g_{n}, e\right)$ is nondecreasing and the averages $A_{N}^{\left(g_{n}\right)} f$ converge a.e. for all $f \in L^{1}(X)$. If $\sum_{j} \beta_{j, M}<\infty$ for every $M<\infty$, then there exists an increasing sequence $\left\{n_{k}\right\} \in \mathbb{N}$ such that $\left\{g_{n_{k}}\right\}$ has gaps tending to infinity, and such that the averages $A_{N}^{\left(g_{n_{k}}\right)} f$ converge a.e. for all $f \in L^{1}(X)$.

Proof. We can clearly choose a sequence $M_{j}$ with $M_{j} \rightarrow \infty$ so that $\sum_{j} \beta_{j, M_{j}}<\infty$. Let $\left\{n_{k}\right\}$ be the set $\mathbb{N} \backslash\left(\bigcup_{j} \Gamma_{j, M_{j}}\right)$, taken in increasing order, and note that $\frac{n_{k}}{k} \rightarrow 1$.

Considering $\left[1, n_{K}\right]$ as the union of terms in $\left\{n_{k}\right\}$ and the complement, we see

$$
\left|A_{K}^{\left(g_{n_{k}}\right)} f(x)-\frac{n_{K}}{K} A_{n_{K}}^{\left(g_{n}\right)} f(x)\right| \leq\left|\frac{1}{K} \sum_{\substack{n<n_{K}: \\ n \in \bigcup_{j} \Gamma_{j, M_{j}}}} f\left(\mathcal{T}\left(g_{n}\right) x\right)\right| .
$$

Clearly the $L^{1}$ norm of the right-hand side is bounded by $\frac{n_{K}-K}{K}\|f\|_{1} \rightarrow 0$ as $K \rightarrow \infty$. Furthermore, we have the weak maximal inequality

$$
\begin{aligned}
\left\|\sup _{K}\left|\frac{1}{K} \sum_{\substack{n<n_{K}: \\
n \in \bigcup_{j} \Gamma_{j, M_{j}}}} f\left(\mathcal{T}\left(g_{n}\right) x\right)\right|\right\| \|_{1} & \leq\left\|\sup _{j} \mid 2^{-j} \sum_{\substack{n<2^{j}: \\
n \in \cup_{j} \Gamma_{j, M_{j}}}} f\left(\mathcal{T}\left(g_{n}\right) x\right)\right\| \|_{j} \\
& \lesssim \sum_{j} \beta_{j, M_{j}}<\infty .
\end{aligned}
$$

Therefore $A_{K}^{\left(g_{n_{k}}\right)} f-\frac{n_{K}}{K} A_{n_{K}}^{\left(g_{n}\right)} f \rightarrow 0$ a.e., and since $\frac{n_{K}}{K} \rightarrow 1$, this proves that $A_{N}^{\left(g_{n_{k}}\right)} f$ converge a.e. for all $f \in L^{1}(X)$.

It remains to show that our randomly generated sequences indeed have this property. If $B$ is the ball of radius $2 M$ centered at $g$, and $\rho(g, e) \approx 2^{l} \gg M$, then clearly

$$
\mathbb{P}\left(\sum_{g \in B} \xi_{g}=t\right) \leq\left(\begin{array}{c}
|B| \\
t
\end{array}\right) 2^{-\gamma t l}
$$


If $\mathcal{B}_{l}$ is a cover of $\left\{g \in G: \rho(g, e) \approx 2^{l}\right\}$ by balls of radius $2 M$, with multiplicity of intersection controlled uniformly in $l$, then for $j=(d-\gamma) l$,

$$
\begin{aligned}
\beta_{j, M} & \lesssim 2^{-j}\left|\mathcal{B}_{l}\right| \sum_{t=2}^{|B|}(t-1)\left(\begin{array}{c}
|B| \\
t
\end{array}\right) 2^{-\gamma t l} \\
& \leq C_{M}\left|\mathcal{B}_{l}\right| 2^{-2 \gamma l-j} \\
& \leq C_{M} 2^{l(d-2 \gamma)-j} \leq C_{M} 2^{-\gamma l},
\end{aligned}
$$

and these are summable.

Remark. Let $\left\{\vec{n}_{i}\right\}$ be one of the sparse deterministic sequences from Section 4 (either speckled or plaid). Because of the nature of the Freiman isomorphism $F_{p}$ in (4.13) and the pseudo-randomness of the points $\left(j, j^{2}, \ldots, j^{m}\right) \in \mathbb{Z}_{p}^{m}$, it becomes vanishingly rare for two points in the $k$ th block to be within $p_{k}$ of each other, and thus $\beta_{j, M}$ are summable as well. Therefore these sequences can be modified in a negligible manner so as to have gaps tending to infinity.

\section{ACKNOWLEDGMEnTs}

The authors thank M. Christ for substantial help on the results in Section 4 , and for disseminating the preprint [10. The first author was partially supported by NSF Grant DMS-0401260 while developing an early version of the results in Section 5 .

\section{REFERENCES}

[1] H. Bass, The degree of polynomial growth of finitely generated nilpotent groups, Proc. London Math. Soc. (3) 25 (1972), 603-614. MR0379672 (52 \#577)

[2] A. Bellow and V. Losert, On sequences of density zero in ergodic theory, Conference in modern analysis and probability (New Haven, Conn., 1982), Contemp. Math., vol. 26, Amer. Math. Soc., Providence, RI, 1984, pp. 49-60, DOI 10.1090/conm/026/737387. MR737387 (86c:28034)

[3] Alexandra Bellow, Roger Jones, and Joseph Rosenblatt, Convergence for moving averages, Ergodic Theory Dynam. Systems 10 (1990), no. 1, 43-62, DOI 10.1017/S0143385700005381. MR.1053798 (91g:28021)

[4] J. Bourgain, An approach to pointwise ergodic theorems, Geometric aspects of functional analysis (1986/87), Lecture Notes in Math., vol. 1317, Springer, Berlin, 1988, pp. 204-223, DOI 10.1007/BFb0081742. MR950982 (90b:28016)

[5] J. Bourgain, On the maximal ergodic theorem for certain subsets of the integers, Israel J. Math. 61 (1988), no. 1, 39-72, DOI 10.1007/BF02776301. MR937581(89f:28037a)

[6] Emmanuel Breuillard. Geometry of locally compact groups of polynomial growth and shape of large balls. Preprint, 2007; revised 2012. arXiv.org/abs/0704.0095

[7] Zoltán Buczolich and R. Daniel Mauldin, Divergent square averages, Ann. of Math. (2) 171 (2010), no. 3, 1479-1530, DOI 10.4007/annals.2010.171.1479. MR2680392 (2011e:37017)

[8] Michael Christ, Weak type (1,1) bounds for rough operators, Ann. of Math. (2) 128 (1988), no. 1, 19-42, DOI 10.2307/1971461. MR951506 (89m:42013)

[9] Michael Christ, A T(b) theorem with remarks on analytic capacity and the Cauchy integral, Colloq. Math. 60/61 (1990), no. 2, 601-628. MR.1096400 (92k:42020)

[10] Michael Christ. A weak type $(1,1)$ inequality for maximal averages over certain sparse sequences. Preprint, 2011. arXiv.org/abs/1108.5664

[11] Charles Fefferman, Inequalities for strongly singular convolution operators, Acta Math. 124 (1970), 9-36. MR0257819 (41 \#2468)

[12] Mikhael Gromov, Groups of polynomial growth and expanding maps, Inst. Hautes Études Sci. Publ. Math. 53 (1981), 53-73. MR623534 (83b:53041) 
[13] Roger L. Jones, Robert Kaufman, Joseph M. Rosenblatt, and Máté Wierdl, Oscillation in ergodic theory, Ergodic Theory Dynam. Systems 18 (1998), no. 4, 889-935, DOI 10.1017/S0143385798108349. MR1645330 (2000b:28019)

[14] Ulrich Krengel, Ergodic theorems, de Gruyter Studies in Mathematics, vol. 6, Walter de Gruyter and Co., Berlin, 1985. With a supplement by Antoine Brunel. MR 797411 (87i:28001)

[15] Patrick LaVictoire, $A n L^{1}$ ergodic theorem for sparse random subsequences, Math. Res. Lett. 16 (2009), no. 5, 849-859. MR2576702 (2011b:60014)

[16] John Milnor, Growth of finitely generated solvable groups, J. Differential Geometry 2 (1968), 447-449. MR0244899(39 \#6212)

[17] Pierre Pansu, Croissance des boules et des géodésiques fermées dans les nilvariétés (French, with English summary), Ergodic Theory Dynam. Systems 3 (1983), no. 3, 415-445, DOI 10.1017/S0143385700002054. MR741395 (85m:53040)

[18] Joseph M. Rosenblatt and Máté Wierdl, Pointwise ergodic theorems via harmonic analysis, Ergodic theory and its connections with harmonic analysis (Alexandria, 1993), London Math. Soc. Lecture Note Ser., vol. 205, Cambridge Univ. Press, Cambridge, 1995, pp. 3-151, DOI 10.1017/CBO9780511574818.002. MR1325697 (96c:28025)

[19] S. Sawyer, Maximal inequalities of weak type, Ann. of Math. (2) 84 (1966), 157-174. MR0209867 (35 \#763)

[20] E. M. Stein, On limits of seqences of operators, Ann. of Math. (2) 74 (1961), 140-170. MR0125392 (23 \#A2695)

[21] Terence Tao and Van Vu, Additive combinatorics, Cambridge Studies in Advanced Mathematics, vol. 105, Cambridge University Press, Cambridge, 2006. MR2289012 (2008a:11002)

[22] A. A. Tempel'man, Ergodic theorems for general dynamical systems (Russian), Dokl. Akad. Nauk SSSR 176 (1967), 790-793. MR0219700 (36 \#2779)

[23] Roman Urban and Jacek Zienkiewicz, Weak type $(1,1)$ estimates for a class of discrete rough maximal functions, Math. Res. Lett. 14 (2007), no. 2, 227-237. MR2318621(2008i:42045)

[24] André Weil, Sur les courbes algébriques et les variétés qui s'en déduisent (French), Actualités Sci. Ind., no. 1041 = Publ. Inst. Math. Univ. Strasbourg 7 (1945), Hermann et Cie., Paris, 1948. MR0027151(10,262c)

[25] Hermann Weyl, Das asymptotische Verteilungsgesetz der Eigenwerte linearer partieller Differentialgleichungen (mit einer Anwendung auf die Theorie der Hohlraumstrahlung) (German), Math. Ann. 71 (1912), no. 4, 441-479, DOI 10.1007/BF01456804. MR1511670

[26] Joseph A. Wolf, Growth of finitely generated solvable groups and curvature of Riemanniann manifolds, J. Differential Geometry 2 (1968), 421-446. MR0248688 (40 \#1939)

Department of Mathematics, University of Wisconsin, Madison, Wisconsin 53706

E-mail address: patlavic@math.wisc.edu

Current address: Principal Engineer, Quixey, 278 Castro Street, Mountain View, California 94041

E-mail address: patrick@quixey.com

Department of Mathematics, University of Illinois at Urbana-Champaign, Urbana, ILLINOIS 61801

E-mail address: ajnparrish@gmail.com

Department of Mathematics, University of Illinois at Urbana-Champaign, Urbana, ILLINOIS 61801

E-mail address: rosnbltt@illinois.edu 